\title{
Characterisation of upper gradients on the weighted Euclidean space and applications
}

\author{
Danka Lučić ${ }^{1}$ Enrico Pasqualetto ${ }^{1} \cdot$ Tapio Rajala $^{1}$ (D)
}

Received: 10 August 2020 / Accepted: 13 February 2021 / Published online: 10 March 2021

(c) The Author(s) 2021

\begin{abstract}
In the context of Euclidean spaces equipped with an arbitrary Radon measure, we prove the equivalence among several different notions of Sobolev space present in the literature and we characterise the minimal weak upper gradient of all Lipschitz functions.
\end{abstract}

Keywords Sobolev space - Weighted Euclidean space $\cdot$ Decomposability bundle

Mathematics Subject Classification $46 \mathrm{E} 35 \cdot 53 \mathrm{C} 23 \cdot 26 \mathrm{~B} 05$

\section{Introduction}

In this paper, we study first-order Sobolev spaces on the Euclidean space $\mathbb{R}^{n}$ equipped with an arbitrary Radon measure $\mu \geq 0$. This theory has been initiated in the late nineties, with the pioneering work [9] by G. Bouchitté, G. Buttazzo, and P. Seppecher. The motivations and applications were numerous, in the fields of calculus of variations [9], shape optimisation [10], optimal transport problems with gradient penalisation [33], among many others. Compared to Allard's theory of varifolds [3] or to Federer-Fleming's theory of currents [21], the usage of measures in optimisation problems presents two main advantages: it allows to model objects made of parts having different Hausdorff dimension (such as multijunctions), and it rests on a solid functional-analytic machinery. About the former feature, we just mention that the aim of [9] was to represent low-dimensional elastic structures (such as membrane and beams) in an intrinsic way, as opposed to the more classical idea of first 'fattening' the structure under consideration and then passing to the limit in the vanishing thickness parameter (via $\Gamma$-convergence methods, for instance). With regard to the latter feature, let us briefly

Tapio Rajala

tapio.m.rajala@jyu.fi

Danka Lučić

danka.lucic@dm.unipi.it

Enrico Pasqualetto

enrico.pasqualetto@sns.it

1 Department of Mathematics and Statistics, University of Jyvaskyla, P.O. Box 35 (MaD), FI-40014 University of Jyvaskyla, Finland 
explain which is the analytic framework the theory of Sobolev spaces on weighted $\mathbb{R}^{n}$ relies upon.

The key idea introduced by [9] was to define a suitable 'tangent distribution' associated with the measure $\mu$, namely a $\mu$-a.e. defined measurable subbundle of $T \mathbb{R}^{n} \cong \mathbb{R}^{n} \times \mathbb{R}^{n}$; see Definition 2.21. In the approach adopted in [9], the tangent fibres are identified by looking at vector fields whose distributional divergence belongs to $L^{2}(\mu)$ (see (2.24) for the precise definition we are referring to). A different (but similar in spirit) notion was studied by Fragalà-Mantegazza [22]; we do not investigate it in this paper. For a complete account on this technique via the distributional divergence, we refer to the survey [11] and the references therein. An alternative way to select the tangent fibres was proposed by Zhikov in $[40,41]$, where the strategy was to perform a relaxation at the level of gradients of smooth functions. We introduce a useful generalisation - called $G$-structure-of Zhikov's concept in Definition 3.8. Later on, J. Louet studied in his PhD thesis [32] the relation between the above two approaches, but their complete equivalence was not known; we will obtain it as a by-product of Theorem 3.16. Once the tangent distribution is given, the Sobolev space is defined by first projecting the gradients of smooth functions on the tangent fibres (obtaining the tangential gradient with respect to $\mu$ ) and then passing to the closure. The resulting energy functional is lower semicontinuous, or equivalently the associated notion of weak gradient yields a closed linear operator. It is worth to recall that other geometric and measure-theoretic notions of tangent space to a measure are studied in the literature-for instance, Preiss' notion of 'tangent measure' [36] or Simon's notion of 'approximate tangent space' [39]. However, these are not the correct objects to look at in order to define a Sobolev space: besides the fact that they not always exist, a noteworthy problem is that the consequent tangential gradient may well be not closable (since the geometric fibres are typically bigger than the analytic ones).

In the present paper, we start our investigation of the Sobolev space on weighted $\mathbb{R}^{n}$ from a rather different viewpoint. More precisely, we regard it as a special case of the more general theory of Sobolev spaces over a metric measure space $(X, \mathrm{~d}, \mu)$. In this respect, the first definition was given by Hajłasz [29], but we will not consider it here because of its 'non-local' nature. At a later time, several other notions (which eventually turned out to be equivalent) have been proposed by Cheeger [13], Shanmugalingam [38], Ambrosio et al. [6], and Di Marino [17]. It will be convenient for us to work with the approach $W^{1,2}(X, \mu)$ based on the concept of test plan, introduced in [6]; see Definition 2.3. The common feature of all the above approaches is the following: in lack of an underlying Banach structure, the weakly differentiable functions $f$ on a metric measure space are detected by estimating the entity of their variation, rather than the variation itself. In other words, one obtains the 'modulus of the weak differential' $\left|D_{\mu} f\right|$ instead of the weak differential $D_{\mu} f$.

Let us focus our attention on the case in which $\mu$ is a Radon measure on $\mathbb{R}^{n}$. Contrarily to what was discussed in the first part of this introduction, we now have a Sobolev space $W^{1,2}\left(\mathbb{R}^{n}, \mu\right)$ at our disposal, but not (a priori) a notion of tangent fibre. Still, a tangent distribution can be recovered by appealing to results available in the literature, as we are going to describe:

- Gigli [24] built an abstract tensor calculus for metric measure spaces $(X, \mathrm{~d}, \mu)$, which is based upon the notion of $L^{2}(\mu)$-normed $L^{\infty}(\mu)$-module. In particular, the Sobolev space gives rise to a natural notion of tangent module $L_{\mu}^{2}(T X)$, whose elements should be regarded as the 'synthetic' vector fields over $(X, \mathrm{~d}, \mu)$. See Definition 2.10. 
- In the framework of the weighted Euclidean space, N. Gigli and the second named author proved in [27] that the tangent module $L_{\mu}^{2}\left(T \mathbb{R}^{n}\right)$ can be isometrically embedded into the space $L^{2}\left(\mathbb{R}^{n}, \mathbb{R}^{n} ; \mu\right)$ of all $L^{2}(\mu)$-maps from $\mathbb{R}^{n}$ to itself. See Theorem 2.16.

- The first and second named authors proved in [34] that (locally finitely generated) $L^{2}(\mu)$ -normed $L^{\infty}(\mu)$-modules can be always represented as the spaces of sections of a measurable Banach bundle. In the specific case of weighted $\mathbb{R}^{n}$, this grants that the tangent module $L_{\mu}^{2}\left(T \mathbb{R}^{n}\right)$ is canonically associated with a distribution $T_{\mu}$ in $\mathbb{R}^{n}$, that we will call the tangent distribution. See Definition 3.4.

One of the main achievements of the present paper is Theorem 3.16, where we prove that the tangent distribution $T_{\mu}$-and accordingly the Sobolev space $W^{1,2}\left(\mathbb{R}^{n}, \mu\right)$-is consistent both with the notion obtained via divergence by Bouchitte-Buttazzo-Seppecher [9] and with the one via vectorial relaxation by Zhikov [40, 41]. Moreover, by building on top of this equivalence result, we will identify the minimal object $\left|D_{\mu} f\right|$ (called the minimal weak upper gradient) associated with any compactly supported Lipschitz function $f$ on $\mathbb{R}^{n}$; see Theorem 3.20. The case $n=1$ was previously investigated by S. Di Marino and G. Speight in [20].

In order to establish the above-mentioned characterisation of the weak gradient of Lipschitz functions, we will need to study the interaction between the Sobolev calculus on weighted $\mathbb{R}^{n}$ and the Alberti-Marchese differentiation theorem [1], which says-roughly speaking - that there exists a maximal distribution $V_{\mu}$ in $\mathbb{R}^{n}$ along which all Lipschitz functions are $\mu$-a.e. differentiable (in the sense of Fréchet); cf. Theorem 3.1. This kind of investigation has been initiated by the first and second named authors together with $\mathrm{S}$. Di Marino in [19], where it is proven that the absolute value of the Alberti-Marchese gradient is a weak upper gradient (see Theorem 3.3). By using the machinery discussed so far, we show (in Corollary 3.17) that

$$
T_{\mu}(x) \subseteq V_{\mu}(x), \quad \text { for } \mu \text {-a.e. } x \in \mathbb{R}^{n} .
$$

However, in general 'Sobolev calculus' and 'Lipschitz calculus' are not equivalent, thus one cannot expect the equality $T_{\mu}=V_{\mu}$ to hold for all measures $\mu$. Indeed, the Alberti-Marchese distribution just depends on the negligible sets of $\mu$, while the Sobolev space $W^{1,2}\left(\mathbb{R}^{n}, \mu\right)$-and thus, a fortiori, the tangent distribution $T_{\mu}$ - strongly depends on the measure $\mu$ itself. An example of a measure $\mu$ on $\mathbb{R}$ for which $T_{\mu} \neq V_{\mu}$ will be described in Remark 3.18.

We are now in a position to state Theorem 3.20: given any $f \in \operatorname{LIP}_{c}\left(\mathbb{R}^{n}\right)$, it holds that

$$
\left|D_{\mu} f\right|=\left|\operatorname{pr}_{T_{\mu}}\left(\nabla_{\mathrm{AM}} f\right)\right|, \quad \text { in the } \mu \text {-a.e. sense, }
$$

where we denote by $\mathrm{pr}_{T_{\mu}}: V_{\mu} \rightarrow T_{\mu}$ the natural projection operator, while $\nabla_{\mathrm{AM}} f$ stands for the Alberti-Marchese gradient of $f$ (that is a measurable section of the distribution $V_{\mu}$ ).

In the last part of the paper-namely in Sect. 4-we shall provide a few applications (for the moment, only at a theoretical level) of our main Theorems 3.16 and 3.20:

- Section 4.1: By combining our techniques with a deep result by G. De Philippis and F. Rindler [15] about Radon measures on $\mathbb{R}^{n}$, we prove that for $\mu^{s}$-a.e. point $x \in \mathbb{R}^{n}$ the tangent fibre $T_{\mu}(x)$ cannot coincide with the whole $\mathbb{R}^{n}$, where $\mu^{s}$ stands for the singular part of $\mu$ with respect to the Lebesgue measure $\mathcal{L}^{n}$; see Theorem 4.6.

- Section 4.2: The tangent distribution $T_{\mu}$ admits a geometric interpretation, in terms of the initial velocities of suitably chosen test plans on $\left(\mathbb{R}^{n}, \mathrm{~d}_{\mathrm{Eucl}}, \mu\right)$; see Theorem 4.16. 
- Section 4.3: Sobolev spaces over the weighted Euclidean space satisfy the expected tensorisation property; see Theorem 4.21 .

We wish to point out that in the whole paper we just stick to the case $p=2$, but mostly for a matter of practicality. The main reason is that many of the tools we will use-those concerning the theory of normed modules-are explicitly written in the literature only for the case $p=2$. However, we expect that our results have appropriate counterparts for every $p \in(1, \infty)$.

Finally, we conclude this introduction by mentioning that also second-order Sobolev spaces on weighted Euclidean spaces (for suitable Radon measures) have been studied, e.g. in [12]. It would be definitely interesting to understand whether even these second-order spaces admit an equivalent reformulation in the language of metric measure spaces. Yet another interesting problem would be to study the space $\mathrm{BV}\left(\mathbb{R}^{n}, \mu\right)$ of functions of bounded variation.

\section{List of symbols}

We provide below a list of the non-standard symbols we shall use throughout the paper.

$\mathcal{L}_{1} \quad$ Restriction of the Lebesgue measure to the interval [0,1]

$\mathrm{d}_{\infty} \quad$ Supremum distance on $C([0,1], X)$

$\mathrm{e}_{t}, \mathrm{e}_{t}^{X} \quad$ Evaluation map at time $t$

$|\dot{\gamma}| \quad$ Metric speed of an absolutely continuous curve $\gamma$

$\mathrm{KE}_{t} \quad$ Kinetic energy functional at time $t$

$\operatorname{lip}(f) \quad$ Local Lipschitz constant of a Lipschitz function $f$

$\operatorname{Comp}(\boldsymbol{\pi}) \quad$ Compression constant of a test plan $\boldsymbol{\pi}$

Const $^{X} \quad$ 'Constant curve' map

$W^{1,2}(X, \mu) \quad$ Sobolev space on a metric measure space $(X, \mathrm{~d}, \mu)$

$\left|D_{\mu} f\right| \quad$ Minimal weak upper gradient of $f \in W^{1,2}(X, \mu)$

$E_{\mathrm{Ch}} \quad$ Cheeger energy functional

$E_{\text {lip }} \quad$ 'Lipschitz' energy functional

$\Delta_{\mu} \quad$ Laplacian operator

$\left\{P_{t}\right\}_{t \geq 0} \quad$ Heat flow semigroup

$\mathrm{R}_{\mathscr{M}} \quad$ Riesz isomorphism associated with a Hilbert module $\mathscr{M}$

$\mathscr{N}^{\perp} \quad$ Orthogonal complement of a submodule $\mathscr{N} \subseteq \mathscr{M}$

$L_{\mu}^{2}\left(T^{*} X\right) \quad$ Abstract cotangent module on $(X, \mathrm{~d}, \mu)$

$\mathrm{d}_{\mu} f \quad$ Abstract differential of a function $f \in W^{1,2}(X, \mu)$

$L_{\mu}^{2}(T X) \quad$ Abstract tangent module on $(X, \mathrm{~d}, \mu)$

$\nabla_{\mu} f \quad$ Abstract gradient of a function $f \in W^{1,2}(X, \mu)$

$\boldsymbol{\pi}_{t}^{\prime} \quad$ Velocity at time $t$ of a test plan $\boldsymbol{\pi}$

$\operatorname{div}_{\mu} \quad$ Abstract divergence operator

Der ${ }_{t} \quad$ 'Derivation' map

$\mathbb{B}_{\pi} \quad$ The space $L^{2}\left(C\left([0,1], \mathbb{R}^{n}\right), \mathbb{R}^{n} ; \boldsymbol{\pi}\right)$

$\mathrm{P}_{\mu} \quad$ 'Projection of 1-forms' map

$l_{\mu} \quad$ 'Embedding of vector fields' map

$\underline{\text { div }}_{\mu} \quad$ Concrete divergence operator

$\overline{\operatorname{Gr}}\left(\mathbb{R}^{n}\right) \quad$ Grassmannian of $\mathbb{R}^{n}$ 
$\mathscr{D}_{n}(\mu) \quad$ Space of distributions on $\mathbb{R}^{n}$ (up to $\mu$-a.e. equality)

$\Gamma(V) \quad$ Space of $L^{2}(\mu)$-sections of a distribution $V \in \mathscr{D}_{n}(\mu)$

$\operatorname{pr}_{V} \quad$ Orthogonal projection map onto $\Gamma(V)$

$V^{\perp} \quad$ Orthogonal complement of a distribution $V \in \mathscr{D}_{n}(\mu)$

$V_{\mu} \quad$ Alberti-Marchese distribution

$\nabla_{\mathrm{AM}} f \quad$ Alberti-Marchese gradient of $f \in \operatorname{LIP}_{c}\left(\mathbb{R}^{n}\right)$

$E_{\mathrm{AM}} \quad$ Alberti-Marchese energy functional

$T_{\mu} \quad$ Tangent distribution

$(\mathcal{V}, \bar{\nabla}) \quad$ An arbitrary $G$-structure

$G_{\mu} \quad$ The $G_{\mu}$-structure $\left(C_{c}^{\infty}\left(\mathbb{R}^{n}\right), \nabla\right)$

$G_{\mathrm{AM}} \quad$ The $G_{\mathrm{AM}}$-structure $\left(\operatorname{LIP}_{c}\left(\mathbb{R}^{n}\right), \nabla_{\mathrm{AM}}\right)$

$G(f) \quad$ The family of $G$-gradients of $f$

$W_{G} \quad$ The unique distribution satisfying $\Gamma\left(W_{G}\right)=G(0)$

$W_{\mu} \quad$ The distribution $W_{G_{\mu}}$

$\mathcal{I}(\underline{v}) \quad$ 'Currentification' of a vector field $\underline{v} \in D\left(\underline{\operatorname{div}}_{\mu}\right)$

$\mathrm{D}_{\pi} \quad$ Initial velocity of a test plan $\pi$

\section{Preliminaries}

\subsection{Sobolev calculus on metric measure spaces}

For the purposes of the present paper, a metric measure space is any triple $(X, \mathrm{~d}, \mu)$, where $(X, \mathrm{~d})$ is a complete and separable metric space, while $\mu \geq 0$ is a boundedly finite Borel measure on $(X, \mathrm{~d})$. We denote by $\mathscr{P}(X)$ the family of all Borel probability measures on $(X, d)$.

\subsubsection{Absolutely continuous curves}

First of all, let us introduce the shorthand notation

$$
\mathcal{L}_{1}:=\left.\mathcal{L}^{1}\right|_{[0,1]}, \quad \text { where } \mathcal{L}^{1} \text { stands for the Lebesgue measure on } \mathbb{R} .
$$

We denote by $C([0,1], X)$ the family of all continuous curves $\gamma:[0,1] \rightarrow X$. It holds that the set $C([0,1], X)$ is a complete and separable metric space when endowed with the supremum distance $d_{\infty}$, which is defined as

$$
\mathrm{d}_{\infty}(\gamma, \sigma):=\max _{t \in[0,1]} \mathrm{d}\left(\gamma_{t}, \sigma_{t}\right), \quad \text { for every } \gamma, \sigma \in C([0,1], X) .
$$

Given any $t \in[0,1]$, we denote by $\mathrm{e}_{t}: C([0,1], X) \rightarrow X$ the evaluation map at time $t$, i.e.

$$
\mathrm{e}_{t}(\gamma)=\mathrm{e}_{t}^{X}(\gamma):=\gamma_{t}, \quad \text { for every } \gamma \in C([0,1], X) .
$$

We say that $\gamma \in C([0,1], X)$ is absolutely continuous if there exists $g \in L^{1}(0,1)$ such that

$$
\mathrm{d}\left(\gamma_{t}, \gamma_{s}\right) \leq \int_{s}^{t} g(r) \mathrm{d} r, \quad \text { for every } s, t \in[0,1] \text { such that } s<t .
$$


The minimal such function $g$ (where minimality is intended in the $\mathcal{L}_{1}$-a.e. sense) is called the metric speed of $\gamma$ and denoted by $|\dot{\gamma}| \in L^{1}(0,1)$. As proven in [4, Theorem 1.1.2], it holds

$$
\left|\dot{\gamma}_{t}\right|=\lim _{h \rightarrow 0} \frac{\mathrm{d}\left(\gamma_{t+h}, \gamma_{t}\right)}{|h|}, \quad \text { for } \mathcal{L}_{1} \text {-a.e. } t \in[0,1]
$$

The family of absolutely continuous curves on $X$ is denoted by $A C([0,1], X)$. Also, we define

$$
A C^{2}([0,1], X):=\left\{\gamma \in A C([0,1], X)|| \dot{\gamma} \mid \in L^{2}(0,1)\right\} .
$$

It is well known that $A C^{2}([0,1], X)$ is a Borel subset of the metric space $\left(C([0,1], X), \mathrm{d}_{\infty}\right)$. Given any $t \in(0,1]$, we define the functional $\mathrm{KE}_{t}: C([0,1], X) \rightarrow[0,+\infty]$ as

$$
\operatorname{KE}_{t}(\gamma):= \begin{cases}t\left(f_{0}^{t}\left|\dot{\gamma}_{s}\right|^{2} \mathrm{~d} s\right)^{1 / 2}, & \text { if } \gamma \in A C^{2}([0,1], X), \\ +\infty & \text { otherwise. }\end{cases}
$$

Given a reflexive, separable Banach space $(\mathbb{B},\|\cdot\|)$ and a curve $\gamma \in A C([0,1], \mathbb{B})$, it holds that $\gamma$ is $\mathcal{L}_{1}$-a.e. differentiable, its $\mathcal{L}_{1}$-a.e. derivative $\dot{\gamma}:[0,1] \rightarrow \mathbb{B}$ is Bochner integrable, and

$$
\gamma_{t}-\gamma_{s}=\int_{s}^{t} \dot{\gamma}_{r} \mathrm{~d} r, \quad \text { for every } s, t \in[0,1] \text { such that } s<t .
$$

Observe that the identity $\left\|\dot{\gamma}_{t}\right\|=\left|\dot{\gamma}_{t}\right|$ is satisfied for $\mathcal{L}_{1}$-a.e. $t \in[0,1]$.

\subsubsection{Lipschitz functions}

The family of all real-valued Lipschitz functions defined on $(X, \mathrm{~d})$ is indicated with $\operatorname{LIP}(X)$. The subfamily of those Lipschitz functions having compact support (resp. bounded support) is denoted by $\operatorname{LIP}_{c}(X)\left(\operatorname{resp} . \operatorname{LIP}_{b s}(X)\right)$. Given any $f \in \operatorname{LIP}(X)$, we define its local Lipschitz constant as

$$
\operatorname{lip}(f)(x):=\varlimsup_{y \rightarrow x} \frac{|f(x)-f(y)|}{\mathrm{d}(x, y)}, \quad \text { whenever } x \in X \text { is an accumulation point, }
$$

and $\operatorname{lip}(f)(x):=0$ elsewhere.

\subsubsection{Sobolev space via test plans}

We recall here the definition of Sobolev space in the metric measure setting and its main properties. The approach we are going to describe has been proposed in $[5,6]$. To begin with, let us recall the important notion of test plan:

Definition 2.1 (Test plan $[5,6])$ Let $(X, \mathrm{~d}, \mu)$ be a metric measure space. Then, we say that a Borel probability measure $\pi$ on $\left(C([0,1], X), \mathrm{d}_{\infty}\right)$ is a test plan on $(X, \mathrm{~d}, \mu)$ provided the following properties are satisfied:

(i) There exists a compression constant $\operatorname{Comp}(\boldsymbol{\pi})>0$ such that 
$\left(\mathrm{e}_{t}\right)_{*} \pi \leq \operatorname{Comp}(\pi) \mu, \quad$ for every $t \in[0,1]$,

where $\left(\mathrm{e}_{t}\right)_{*} \pi$ stands for the pushforward measure of $\boldsymbol{\pi}$ under the evaluation map $\mathrm{e}_{t}$.

(ii) The measure $\pi$ is concentrated on $A C^{2}([0,1], X)$ and has finite kinetic energy, i.e.

$$
\int \mathrm{KE}_{1}(\gamma)^{2} \mathrm{~d} \boldsymbol{\pi}(\gamma)=\iint_{0}^{1}\left|\dot{\gamma}_{t}\right|^{2} \mathrm{~d} t \mathrm{~d} \boldsymbol{\pi}(\gamma)<+\infty .
$$

Example 2.2 Given a metric measure space $(X, \mathrm{~d}, \mu)$, we set Const $^{X}: X \rightarrow C([0,1], X)$ as

$$
\operatorname{Const}^{X}(x)_{t}:=x, \quad \text { for every } x \in X \text { and } t \in[0,1] .
$$

Then, the map Const ${ }^{X}$ is an isometry and the measure $\pi:=\operatorname{Const}_{*}^{X} \nu$ is a test plan on $(X, \mathrm{~d}, \mu)$ for every $\nu \in \mathscr{P}(X)$ satisfying $\nu \leq C \mu$ for some constant $C>0$.

The notion of test plan plays an essential role in the definition of Sobolev space:

Definition 2.3 (Sobolev space via test plans $[5,6])$ Let $(X, \mathrm{~d}, \mu)$ be a metric measure space. Fix $f \in L^{2}(\mu)$. Then, a function $G \in L^{2}(\mu)$ is said to be a weak upper gradient of $f$ provided for any test plan $\pi$ on $(X, \mathrm{~d}, \mu)$ the following property is satisfied: for $\pi$-a.e. $\gamma$, it holds that $f \circ \gamma \in W^{1,1}(0,1)$ and

$$
\left|(f \circ \gamma)_{t}^{\prime}\right| \leq G\left(\gamma_{t}\right)\left|\dot{\gamma}_{t}\right|, \quad \text { for } \mathcal{L}_{1} \text {-a.e. } t \in[0,1]
$$

We define the Sobolev space $W^{1,2}(X, \mu)$ as the family of all those functions $f \in L^{2}(\mu)$ that admit a weak upper gradient. Given any $f \in W^{1,2}(X, \mu)$, we denote by $\left|D_{\mu} f\right|$ the minimal weak upper gradient of $f$, where minimality is intended in the $\mu$-a.e. sense.

The original notion of Sobolev space $W^{1,2}(X, \mu)$ via test plans has been introduced in [6], but its equivalent reformulation we presented above has been established in [23, Appendix B]. We chose the unusual notation $W^{1,2}(X, \mu)$, where the distance d does not appear (even though it plays a role in the definition), for a matter of practicality, since in all the cases we shall consider, the distance — differently from the measure-will always remain fixed.

Given any function $f \in \operatorname{LIP}_{b s}(X)$, it holds that $f \in W^{1,2}(X, \mu)$ and

$$
\left|D_{\mu} f\right| \leq \operatorname{lip}(f), \quad \mu \text {-a.e. on } X \text {. }
$$

The equality in (2.8) is achieved only in particular circumstances; see, e.g. Corollary 3.21 and Remark 4.10.

\subsubsection{Energy functionals}

Throughout the whole paper, we will consider several different energy functionals $E: L^{2}(\mu) \rightarrow[0,+\infty]$ over a given metric measure space $(X, \mathrm{~d}, \mu)$. Let us fix some notation. The finiteness domain of $\mathrm{E}$ is given by $D(E):=\left\{f \in L^{2}(\mu): E(f)<+\infty\right\}$. We say that $\mathrm{E}$ is 2-homogeneous provided $E(\lambda f)=\lambda^{2} E(f)$ for every $f \in D(E)$ and $\lambda \in \mathbb{R}$, while it is convex provided $E(\lambda f+(1-\lambda) g) \leq \lambda E(f)+(1-\lambda) E(g)$ for every $f, g \in L^{2}(\mu)$ and $\lambda \in[0,1]$. The functional $\mathrm{E}$ is said to satisfy the parallelogram rule if it holds that 


$$
E(f+g)+E(f-g)=2 E(f)+2 E(g), \quad \text { for every } f, g \in D(E) .
$$

Moreover, we say that the functional $\mathrm{E}$ is lower semicontinuous provided

$$
E(f) \leq \lim _{n \rightarrow \infty} E\left(f_{n}\right), \quad \text { for every } f, f_{n} \in L^{2}(\mu) \text { such that } f_{n} \rightarrow f \text { in } L^{2}(\mu) .
$$

The lower semicontinuous envelope $\tilde{E}: L^{2}(\mu) \rightarrow[0,+\infty]$ of $\mathrm{E}$ is defined as

$$
\tilde{E}(f):=\inf \underline{\lim }_{n \rightarrow \infty} E\left(f_{n}\right), \quad \text { for every } f \in L^{2}(\mu),
$$

where the infimum is taken among all sequences $\left(f_{n}\right)_{n} \subseteq L^{2}(\mu)$ such that $f_{n} \rightarrow f$ in $L^{2}(\mu)$. It holds that $\tilde{E}$ is the greatest lower semicontinuous functional which is dominated by E.

The most important energy functional we will consider is the so-called Cheeger energy:

Definition 2.4 (Cheeger energy) Let $(X, \mathrm{~d}, \mu)$ be a metric measure space. Then, we define

$$
E_{\mathrm{Ch}}(f):= \begin{cases}\frac{1}{2} \int\left|D_{\mu} f\right|^{2} \mathrm{~d} \mu, & \text { if } f \in W^{1,2}(X, \mu), \\ +\infty, & \text { otherwise. }\end{cases}
$$

The functional $E_{\mathrm{Ch}}: L^{2}(\mu) \rightarrow[0,+\infty]$ is called the Cheeger energy associated with $(X, \mathrm{~d}, \mu)$.

The map $E_{\mathrm{Ch}}$ is convex, 2-homogeneous, and lower semicontinuous. Also, $f \mapsto \sqrt{2 E_{\mathrm{Ch}}(f)}$ is a seminorm on $D\left(E_{\mathrm{Ch}}\right)=W^{1,2}(X, \mu)$. In particular, $W^{1,2}(X, \mu)$ is a Banach space if endowed with the following norm:

$$
\|f\|_{W^{1,2}(X, \mu)}:=\left(\|f\|_{L^{2}(\mu)}^{2}+2 E_{\mathrm{Ch}}(f)\right)^{1 / 2}, \quad \text { for every } f \in W^{1,2}(X, \mu) .
$$

Another energy functional to take into account is the following one:

$$
E_{\text {lip }}(f):= \begin{cases}\frac{1}{2} \int \operatorname{lip}^{2}(f) \mathrm{d} \mu, & \text { if } f \in \operatorname{LIP}_{b s}(X), \\ +\infty, & \text { otherwise. }\end{cases}
$$

In view of (2.8), we know that $E_{\mathrm{Ch}} \leq E_{\text {lip. }}$. Actually, $E_{\mathrm{Ch}}$ is the lower semicontinuous envelope of $E_{\text {lip }}$, as granted by the following important result.

Theorem 2.5 (Density in energy of Lipschitz functions [5]) Let $(X, \mathrm{~d}, \mu)$ be a metric measure space. Let $f \in W^{1,2}(X, \mu)$ be given. Then, there exists $\left(f_{n}\right)_{n} \subseteq \operatorname{LIP}_{b s}(X)$ such that $f_{n} \rightarrow f$ and $\operatorname{lip}\left(f_{n}\right) \rightarrow\left|D_{\mu} f\right|$ in $L^{2}(\mu)$.

\subsubsection{Infinitesimal Hilbertianity}

The following definition has been introduced in [23]:

Definition 2.6 (Infinitesimal Hilbertianity) A metric measure space $(X, \mathrm{~d}, \mu)$ is said to be infinitesimally Hilbertian provided the Sobolev space $W^{1,2}(X, \mu)$ is Hilbert. Equivalently, if the Cheeger energy $E_{\mathrm{Ch}}$ satisfies the parallelogram rule. 
Given an infinitesimally Hilbertian space $(X, \mathrm{~d}, \mu)$, it holds that the mapping

$$
\left\langle\nabla_{\mu} f, \nabla_{\mu} g\right\rangle:=\frac{\left|D_{\mu}(f+g)\right|^{2}-\left|D_{\mu} f\right|^{2}-\left|D_{\mu} g\right|^{2}}{2}, \quad \mu \text {-a.e. on } X,
$$

defines a symmetric, bilinear form on $W^{1,2}(X, \mu) \times W^{1,2}(X, \mu)$ with values in $L^{1}(\mu)$.

\subsubsection{Laplacian and heat flow}

Let $(X, \mathrm{~d}, \mu)$ be an infinitesimally Hilbertian space. Given any function $f \in W^{1,2}(X, \mu)$, we declare that $f \in D\left(\Delta_{\mu}\right)$ if there exists $h \in L^{2}(\mu)$ such that

$$
\int g h \mathrm{~d} \mu=-\int\left\langle\nabla_{\mu} f, \nabla_{\mu} g\right\rangle \mathrm{d} \mu, \quad \text { for every } g \in W^{1,2}(X, \mu) .
$$

Since $h$ is uniquely determined, we denote it by $\Delta_{\mu} f$ and call it the Laplacian of $f$. It holds that $D\left(\Delta_{\mu}\right)$ is a linear subspace of $W^{1,2}(X, \mu)$ and $\Delta_{\mu}: D\left(\Delta_{\mu}\right) \rightarrow L^{2}(\mu)$ is a linear operator.

The heat flow $\left\{P_{t}\right\}_{t \geq 0}$ on $(X, \mathrm{~d}, \mu)$ is defined as follows: for any given function $f \in L^{2}(\mu)$, we have that $[0,+\infty) \ni t \mapsto P_{t} f \in L^{2}(\mu)$ is the unique continuous curve satisfying $P_{0} f=f$, which is absolutely continuous on $(0,+\infty)$, such that $P_{t} f \in D\left(\Delta_{\mu}\right)$ holds for all $t>0$ and

$$
\frac{\mathrm{d}}{\mathrm{d} t} P_{t} f=\Delta_{\mu} P_{t} f, \quad \text { for } \mathcal{L}^{1} \text {-a.e. } t>0
$$

Given any function $f \in W^{1,2}(X, \mu)$, it holds that

$$
\left\|P_{t} f\right\|_{W^{1,2}(X, \mu)} \leq\|f\|_{W^{1,2}(X, \mu)}, \quad \text { for every } t>0 .
$$

The above properties are ensured by the classical Komura-Brezis theory of gradient flows.

\subsection{Differential structure of metric measure spaces}

A first-order differential calculus on metric measure spaces has been developed in [24, 25]. Let us briefly recall the key concepts.

\subsubsection{The theory of normed modules}

Let $(X, \mathrm{~d}, \mu)$ be a given metric measure space. Let $\mathscr{M}$ be an algebraic module over the commutative ring $L^{\infty}(\mu)$. Then, a pointwise norm on $\mathscr{M}$ is a mapping $|\cdot|: \mathscr{M} \rightarrow L^{2}(\mu)$ satisfying the following properties:

$$
\begin{aligned}
|v| \geq 0, & \text { for every } v \in \mathscr{M}, \text { with equality if and only if } v=0, \\
|v+w| \leq|v|+|w|, & \text { for every } v, w \in \mathscr{M}, \\
|f v|=|f||v|, & \text { for every } f \in L^{\infty}(\mu) \text { and } v \in \mathscr{M} .
\end{aligned}
$$

(All inequalities are intended in the $\mu$-a.e. sense.) We say that $(\mathscr{M},|\cdot|)$, or just $\mathscr{M}$, is an $L^{2}(\mu)$-normed $L^{\infty}(\mu)$-module provided the norm $\|v\|_{\mathscr{M}}:=\||v|\|_{L^{2}(\mu)}$ on $\mathscr{M}$ is complete.

By a morphism $\varphi: \mathscr{M} \rightarrow \mathscr{N}$ between two given $L^{2}(\mu)$-normed $L^{\infty}(\mu)$-modules $\mathscr{M}, \mathscr{N}$ we mean an $L^{\infty}(\mu)$-linear and continuous map. The dual module $\mathscr{M}^{*}$ of $\mathscr{M}$ is defined as the space of all $L^{\infty}(\mu)$-linear and continuous maps from $\mathscr{M}$ to $L^{1}(\mu)$. It holds that $\mathscr{M}^{*}$ has a natural $L^{2}(\mu)$-normed $L^{\infty}(\mu)$-module structure, the pointwise norm $|L|$ of $L \in \mathscr{M}^{*}$ 
being defined as the minimal function $G \in L^{2}(\mu)$, where minimality is intended in the $\mu$ -a.e. sense, such that the inequality $|L(v)| \leq G|v|$ is satisfied $\mu$-a.e. on $X$ for every element $v \in \mathscr{M}$.

By a Hilbert module on $(X, \mathrm{~d}, \mu)$ we mean an $L^{2}(\mu)$-normed $L^{\infty}(\mu)$-module $\mathscr{M}$ such that

$$
|v+w|^{2}+|v-w|^{2}=2|v|^{2}+2|w|^{2} \quad \mu \text {-a.e., } \quad \text { for every } v, w \in \mathscr{M} \text {. }
$$

Clearly, $\mathscr{M}$ is a Hilbert module if and only if it is Hilbert when viewed as a Banach space. Given two elements $v, w \in \mathscr{M}$, we define their pointwise scalar product $\langle v, w\rangle \in L^{1}(\mu)$ as

$$
\langle v, w\rangle:=\frac{|v+w|^{2}-|v|^{2}-|w|^{2}}{2}, \quad \text { in the } \mu \text {-a.e. sense. }
$$

The resulting mapping $\langle\cdot, \cdot\rangle: \mathscr{M} \times \mathscr{M} \rightarrow L^{1}(\mu)$ is $L^{\infty}(\mu)$-bilinear and symmetric. It holds that the morphism $\mathrm{R}_{\mathscr{M}}: \mathscr{M} \rightarrow \mathscr{M}^{*}$ of $L^{2}(\mu)$-normed $L^{\infty}(\mu)$-modules defined as

$$
\mathrm{R}_{\mathscr{M}}(v)(w):=\langle v, w\rangle \in L^{1}(\mu), \quad \text { for every } v, w \in \mathscr{M},
$$

is an isometric isomorphism. We call $\mathrm{R}_{\mathscr{M}}$ the Riesz isomorphism associated with $\mathscr{M}$.

Remark 2.7 (Orthogonal complement, I) Let $\mathscr{M}$ be a Hilbert module on $(X, \mathrm{~d}, \mu)$. Then, we define the orthogonal complement of a given submodule $\mathscr{N} \subseteq \mathscr{M}$ as

$$
\mathscr{N}^{\perp}:=\{v \in \mathscr{M} \mid\langle v, w\rangle=0 \text { in the } \mu \text {-a.e. sense, for every } w \in \mathscr{N}\} .
$$

Then, $\mathscr{N}^{\downarrow}$ is a submodule of $\mathscr{M}$ that satisfies $\mathscr{N} \cap \mathscr{N}^{\downarrow}=\{0\}$ and $\mathscr{N}+\mathscr{N}^{\downarrow}=\mathscr{M}$.

Definition 2.8 (Dimension of a normed module [24, Section 1.4]) Let $(X, \mathrm{~d}, \mu)$ be a metric measure space. Let $\mathscr{M}$ be an $L^{2}(\mu)$-normed $L^{\infty}(\mu)$-module and let $E \subseteq X$ be a Borel set such that $\mu(E)>0$. Then:

(i) We say that some elements $v_{1}, \ldots, v_{n} \in \mathscr{M}$ are independent on the set $E$ provided the mapping $L^{\infty}\left(\left.\mu\right|_{E}\right)^{n} \ni\left(f_{1}, \ldots, f_{n}\right) \mapsto \sum_{i=1}^{n} f_{i} v_{i} \in \mathscr{M}$ is injective.

(ii) A family $\mathscr{F} \subseteq \mathscr{M}$ is said to generate $\mathscr{M}$ on $E$ provided the linear space $\mathcal{V}$, given by

$$
\mathcal{V}:=\left\{\sum_{i=1}^{n} f_{i} v_{i} \mid n \in \mathbb{N},\left(f_{i}\right)_{i=1}^{n} \subseteq L^{\infty}\left(\left.\mu\right|_{E}\right),\left(v_{i}\right)_{i=1}^{n} \subseteq \mathscr{F}\right\} \subseteq \mathscr{M},
$$

is dense in the restricted module $\mathscr{M}_{E}:=\left\{\mathbb{1}_{E} v: v \in \mathscr{M}\right\}$.

We say that $\mathscr{M}$ has dimension $n \in \mathbb{N}$ on $E$ provided it admits a local basis $v_{1}, \ldots, v_{n} \in \mathscr{M}$ on $E$, i.e. the elements $v_{1}, \ldots, v_{n}$ are independent on $E$ and $\left\{v_{1}, \ldots, v_{n}\right\}$ generates $\mathscr{M}$ on $E$.

Let $\left(X, \mathrm{~d}_{X}, \mu\right),\left(Y, \mathrm{~d}_{Y}, v\right)$ be metric measure spaces. Let $\varphi: X \rightarrow Y$ be a given Borel map. Then, we say that $\varphi$ is a map of bounded compression provided $\varphi_{*} \mu \leq C \nu$ for some $C>0$.

Theorem 2.9 (Pullback module [25, Section 1.4.1]) Let $\left(X, \mathrm{~d}_{X}, \mu\right),\left(Y, \mathrm{~d}_{Y}, v\right)$ be two metric measure spaces. Let $\mathscr{M}$ be an $L^{2}(v)$-normed $L^{\infty}(v)$-module and $\varphi: X \rightarrow Y$ a map of bounded compression. Then, there exists a unique couple $\left(\varphi^{*} \mathscr{M}, \varphi^{*}\right)$, where $\varphi^{*} \mathscr{M}$ is an $L^{2}(\mu)$-normed $L^{\infty}(\mu)$-module called the pullback module and $\varphi^{*}: \mathscr{M} \rightarrow \varphi^{*} \mathscr{M}$ is a linear 
operator called the pullback map, such that $\left|\varphi^{*} v\right|=|v| \circ \varphi$ holds $\mu$-a.e. for all $v \in \mathscr{M}$ and $\left\{\varphi^{*} v: v \in \mathscr{M}\right\}$ generates $\varphi^{*} \mathscr{M}$ on $X$.

Moreover, given two $L^{2}(v)$-normed $L^{\infty}(v)$-modules $\mathscr{M}, \mathscr{N}$ and a morphism $\Phi: \mathscr{M} \rightarrow \mathscr{N}$, there is a unique morphism $\varphi^{*} \Phi: \varphi^{*} \mathscr{M} \rightarrow \varphi^{*} \mathscr{N}$ of $L^{2}(\mu)$-normed $L^{\infty}(\mu)$ -modules such that

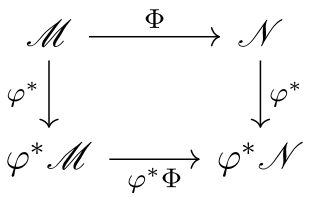

is a commutative diagram.

\subsubsection{Abstract 1-forms and vector fields}

The language of normed modules discussed in the previous section can be used to provide abstract notions of 1-forms and vector fields - tightly linked to the Sobolev calculus-on general metric measure spaces:

Theorem 2.10 (Cotangent and tangent modules [25, Sections 1.2.2 and 1.3.2]) Let $(X, \mathrm{~d}, \mu)$ be a metric measure space. Then, there exists a unique couple $\left(L_{\mu}^{2}\left(T^{*} X\right), \mathrm{d}_{\mu}\right)$, where the cotangent module $L_{\mu}^{2}\left(T^{*} X\right)$ is an $L^{2}(\mu)$-normed $L^{\infty}(\mu)$-module and the differential

$$
\mathrm{d}_{\mu}: W^{1,2}(X, \mu) \longrightarrow L_{\mu}^{2}\left(T^{*} X\right)
$$

is a linear operator, such that the following properties are satisfied:

$$
\begin{array}{ll}
\left|\mathrm{d}_{\mu} f\right|=\left|D_{\mu} f\right| \quad \mu \text {-a.e., } & \text { for every } f \in W^{1,2}(X, \mu), \\
\left\{\mathrm{d}_{\mu} f \mid f \in W^{1,2}(X, \mu)\right\} & \text { generates } L_{\mu}^{2}\left(T^{*} X\right) \text { on } X .
\end{array}
$$

Moreover, if $(X, \mathrm{~d}, \mu)$ is infinitesimally Hilbertian, then $L_{\mu}^{2}\left(T^{*} X\right)$ is a Hilbert module and the tangent module is defined as $L_{\mu}^{2}(T X):=L_{\mu}^{2}\left(T^{*} X\right)^{*}$. The gradient $\nabla_{\mu} f \in L_{\mu}^{2}(T X)$ of a function $f \in W^{1,2}(X, \mu)$ is given by the image of $\mathrm{d}_{\mu} f$ under the Riesz isomorphism $\mathrm{R}_{L_{\mu}^{2}\left(T^{*} X\right)}$.

It holds that a given metric measure space $(X, d, \mu)$ is infinitesimally Hilbertian if and only if its associated modules $L_{\mu}^{2}\left(T^{*} X\right)$ and $L_{\mu}^{2}(T X)$ are Hilbert.

Proposition 2.11 (Closure of the differential [24, Theorem 2.2.9]) Let $(X, \mathrm{~d}, \mu)$ be a metric measure space. Let $\left(f_{n}\right)_{n} \subseteq W^{1,2}(X, \mu)$ satisfy $f_{n} \rightarrow f$ weakly in $L^{2}(\mu)$ for some $f \in L^{2}(\mu)$ and $\mathrm{d}_{\mu} f_{n} \rightarrow \omega$ weakly in $L_{\mu}^{2}\left(T^{*} X\right)$ for some $\omega \in L_{\mu}^{2}\left(T^{*} X\right)$. Then $f \in W^{1,2}(X, \mu)$ and $\mathrm{d}_{\mu} f=\omega$

Given a test plan $\pi$ on a metric measure space $(X, \mathrm{~d}, \mu)$, it holds that for every $t \in[0,1]$ the evaluation map $\mathrm{e}_{t}$ is a map of bounded compression between $(C([0,1], X), \pi)$ and $(X, \mu)$. This allows us to consider the pullback modules $\mathrm{e}_{t}^{*} L_{\mu}^{2}\left(T^{*} X\right)$ and $\mathrm{e}_{t}^{*} L_{\mu}^{2}(T X)$. 
Proposition 2.12 (Velocity of a test plan [24, Theorem 2.3.18]) Let $(X, \mathrm{~d}, \mu)$ be a metric measure space such that the module $L_{\mu}^{2}(T X)$ is separable. Let $\pi$ be a test plan on $(X, \mathrm{~d}, \mu)$. Then, for $\mathcal{L}_{1}$-a.e. $t \in[0,1]$ there exists a unique element $\pi_{t}^{\prime} \in \mathrm{e}_{t}^{*} L_{\mu}^{2}(T X)$, called the velocity of $\boldsymbol{\pi}$ at $t$, such that

$$
\lim _{h \rightarrow 0}\left\|\frac{f \mathrm{oe}_{t+h}-f \circ \mathrm{e}_{t}}{h}-\left(\mathrm{e}_{t}^{*} \mathrm{~d}_{\mu} f\right)\left(\pi_{t}^{\prime}\right)\right\|_{L^{1}(\pi)}=0, \quad \text { for every } f \in W^{1,2}(X, \mu) .
$$

Moreover, it holds that $\left|\pi_{t}^{\prime}\right|(\gamma)=\left|\dot{\gamma}_{t}\right|$ for $\left(\pi \otimes \mathcal{L}_{1}\right)$-a.e. $(\gamma, t) \in A C^{2}([0,1], X) \times[0,1]$.

\subsubsection{Divergence of abstract vector fields}

Let $(X, \mathrm{~d}, \mu)$ be an infinitesimally Hilbertian space. We declare that $v \in L_{\mu}^{2}(T X)$ belongs to $D\left(\operatorname{div}_{\mu}\right)$ provided there exists $h \in L^{2}(\mu)$ such that

$$
\int \mathrm{d}_{\mu} f(v) \mathrm{d} \mu=-\int f h \mathrm{~d} \mu, \quad \text { for every } f \in W^{1,2}(X, \mu) .
$$

The uniquely determined function $h$ will be denoted by $\operatorname{div}_{\mu}(v)$ and called the abstract divergence of $v$. It can be readily checked that a function $f \in W^{1,2}(X, \mu)$ belongs to $D\left(\Delta_{\mu}\right)$ if and only if $\nabla_{\mu} f \in D\left(\operatorname{div}_{\mu}\right)$. In this case, it also holds that $\operatorname{div}_{\mu}\left(\nabla_{\mu} f\right)=\Delta_{\mu} f$.

Let $f \in \operatorname{LIP}_{b s}(X)$ and $v \in D\left(\operatorname{div}_{\mu}\right)$ be given. Then, it holds that $f v \in D\left(\operatorname{div}_{\mu}\right)$ and

$$
\operatorname{div}_{\mu}(f v)=f \operatorname{div}_{\mu}(v)+\left\langle\nabla_{\mu} f, v\right\rangle, \quad \mu \text {-a.e. on } X .
$$

In other words, we say that the abstract divergence satisfies the Leibniz rule.

Lemma 2.13 (Density of vector fields with divergence) Let $(X, \mathrm{~d}, \mu)$ be an infinitesimally Hilbertian space. Then $D\left(\Delta_{\mu}\right)$ is dense in $W^{1,2}(X, \mu)$ and $D\left(\operatorname{div}_{\mu}\right)$ is dense in $L_{\mu}^{2}(T X)$.

Proof First of all, fix $f \in W^{1,2}(X, \mu)$ and consider $P_{t} f \in D\left(\Delta_{\mu}\right)$ for every $t>0$. Since the family $\left\{P_{t} f\right\}_{t>0} \subseteq W^{1,2}(X, \mu)$ is bounded by (2.13) and $W^{1,2}(X, \mu)$ is reflexive, there exists a sequence $t_{n} \searrow 0$ such that $P_{t_{n}} f \rightarrow f$ weakly in $W^{1,2}(X, \mu)$. By Banach-Saks theorem, we have that (possibly passing to a not relabelled subsequence) the sequence $\left(f_{n}\right)_{n} \subseteq D\left(\Delta_{\mu}\right)$ given by $f_{n}:=\frac{1}{n} \sum_{i=1}^{n} P_{t_{i}} f$ satisfies $f_{n} \rightarrow f$ with respect to the strong topology of $W^{1,2}(X, \mu)$

In order to prove the last part of the statement, fix $v \in L_{\mu}^{2}(T X)$ and $\varepsilon>0$. We can find functions $f_{1}^{\prime}, \ldots, f_{n}^{\prime} \in W^{1,2}(X, \mu)$ and $g_{1}^{\prime}, \ldots, g_{n}^{\prime} \in L^{\infty}(\mu)$ with $\left\|v-\sum_{i=1}^{n} g_{i}^{\prime} \nabla_{\mu} f_{i}^{\prime}\right\|_{L_{\mu}^{2}(T X)}<\frac{\varepsilon}{2}$. Thanks to the first part of the statement and the fact that boundedly supported Lipschitz functions are weakly dense in $L^{\infty}(\mu)$, there are $f_{1}, \ldots, f_{n} \in D\left(\Delta_{\mu}\right)$ and $g_{1}, \ldots, g_{n} \in \operatorname{LIP}_{b s}(X)$ such that $\left\|\sum_{i=1}^{n}\left(g_{i}^{\prime} \nabla_{\mu} f_{i}^{\prime}-g_{i} \nabla_{\mu} f_{i}\right)\right\|_{L_{\mu}^{2}(T X)}<\frac{\varepsilon}{2}$. Consequently, we conclude that the vector field $w:=\sum_{i=1}^{n} g_{i} \nabla_{\mu} f_{i}$, which belongs to $D\left(\operatorname{div}_{\mu}\right)$ by (2.17), satisfies $\|v-w\|_{L_{\mu}^{2}(T X)}<\varepsilon$. 


\subsubsection{Concrete 1-forms and vector fields on weighted $\mathbb{R}^{n}$}

Let $(X, \mathrm{~d}, \mu)$ be a metric measure space and $(\mathbb{B},\|\cdot\|)$ a separable Banach space. Then, we denote by $L^{2}(X, \mathbb{B} ; \mu)$ the family of all Borel maps $v: X \rightarrow \mathbb{B}$ such that $\int\|v(x)\|^{2} \mathrm{~d} \mu(x)<+\infty$, considered up to $\mu$-a.e. equality. It holds that $L^{2}(X, \mathbb{B} ; \mu)$ is an $L^{2}(\mu)$-normed $L^{\infty}(\mu)$-module when endowed with the natural pointwise operations and the following pointwise norm: given any $v \in L^{2}(X, \mathbb{B} ; \mu)$, we define

$$
|v|(x):=\|v(x)\|, \quad \text { for } \mu \text {-a.e. } x \in X .
$$

Moreover, it holds (assuming $\mu \neq 0$ ) that $L^{2}(X, \mathbb{B} ; \mu)$ is Hilbert if and only if $\mathbb{B}$ is Hilbert.

We denote by $d_{\text {Eucl }}$ the Euclidean distance $d_{\text {Eucl }}(x, y):=|x-y|$ on $\mathbb{R}^{n}$. Given any $t \in[0,1]$, we define the mapping $\operatorname{Der}_{t}: C\left([0,1], \mathbb{R}^{n}\right) \rightarrow \mathbb{R}^{n}$ as

$$
\operatorname{Der}_{t}(\gamma):= \begin{cases}\dot{\gamma}_{t}, & \text { if } \dot{\gamma}_{t}=\lim _{h \rightarrow 0} \frac{\gamma_{t+h}-\gamma_{t}}{h} \text { exists } \\ 0, & \text { otherwise. }\end{cases}
$$

Standard arguments show that Der ${ }_{t}$ is Borel. Given a non-negative Radon measure $\mu$ on $\mathbb{R}^{n}$ and a test plan $\pi$ on $\left(\mathbb{R}^{n}, \mathrm{~d}_{\mathrm{Eucl}}, \mu\right)$, we define the space $\mathbb{B}_{\boldsymbol{\pi}}$ as

$$
\mathbb{B}_{\pi}:=L^{2}\left(C\left([0,1], \mathbb{R}^{n}\right), \mathbb{R}^{n} ; \boldsymbol{\pi}\right)
$$

Observe that $\mathbb{B}_{\boldsymbol{\pi}}$ is a separable Hilbert space.

Proposition 2.14 Let $\mu$ be a Radon measure on $\mathbb{R}^{n}$. Let $\boldsymbol{\pi}$ be a test plan on $\left(\mathbb{R}^{n}, \mathrm{~d}_{\mathrm{Eucl}}, \mu\right)$. Then, it holds that (the equivalence classes up to $\mathcal{L}_{1}$-a.e. equality of) the mappings

$$
\begin{aligned}
\mathrm{e}-\mathrm{e}_{0}:[0,1] \longrightarrow \mathbb{B}_{\pi}, \quad t \longmapsto \mathrm{e}_{t}-\mathrm{e}_{0}, \\
\text { Der : }[0,1] \longrightarrow \mathbb{B}_{\pi}, \quad t \longmapsto \operatorname{Der}_{t},
\end{aligned}
$$

belong to the space $L^{2}\left([0,1], \mathbb{B}_{\pi} ; \mathcal{L}_{1}\right)$. Moreover, we have that $\mathrm{e}-\mathrm{e}_{0} \in A C^{2}\left([0,1], \mathbb{B}_{\pi}\right)$ and

$$
\frac{\mathrm{d}}{\mathrm{d} t}\left(\mathrm{e}_{t}-\mathrm{e}_{0}\right)=\operatorname{Der}_{t}, \quad \text { for } \mathcal{L}_{1} \text {-a.e. } t \in[0,1],
$$

where the derivative is intended with respect to the strong topology of $\mathbb{B}_{\pi}$.

Proof First of all, let us observe that

$$
\int_{0}^{1}\left(\int\left|\operatorname{Der}_{t}\right|^{2} \mathrm{~d} \boldsymbol{\pi}\right) \mathrm{d} t=\iint_{0}^{1}\left|\dot{\gamma}_{t}\right|^{2} \mathrm{~d} t \mathrm{~d} \boldsymbol{\pi}(\gamma)<+\infty,
$$

thus $\operatorname{Der}_{t} \in \mathbb{B}_{\pi}$ for $\mathcal{L}_{1}$-a.e. $t \in[0,1]$ and $\operatorname{Der} \in L^{2}\left([0,1], \mathbb{B}_{\pi} ; \mathcal{L}_{1}\right)$; we omit the standard proof of the fact that Der is Borel. Moreover, for every $s, t \in[0,1]$ with $s<t$ it holds

$$
\left(\mathrm{e}_{t}-\mathrm{e}_{s}\right)(\gamma)=\gamma_{t}-\gamma_{s}=\int_{s}^{t} \dot{\gamma}_{r} \mathrm{~d} r=\left(\int_{s}^{t} \operatorname{Der}_{r} \mathrm{~d} r\right)(\gamma), \quad \text { for } \pi \text {-a.e. } \gamma,
$$

so that $\left(\mathrm{e}_{t}-\mathrm{e}_{0}\right)-\left(\mathrm{e}_{s}-\mathrm{e}_{0}\right)=\mathrm{e}_{t}-\mathrm{e}_{s}=\int_{s}^{t} \operatorname{Der}_{r} \mathrm{~d} r \in \mathbb{B}_{\pi}$, whence the statement follows. 
Given any non-negative Radon measure $\mu$ on $\mathbb{R}^{n}$, we will refer to the metric measure space $\left(\mathbb{R}^{n}, \mathrm{~d}_{\mathrm{Eucl}}, \mu\right)$ as a weighted Euclidean space. The rest of this paper is devoted to the study of the Sobolev space and the differential structure associated with $\left(\mathbb{R}^{n}, \mathrm{~d}_{\text {Eucl }}, \mu\right)$. We will refer to the elements of the Hilbert module $L^{2}\left(\mathbb{R}^{n}, \mathbb{R}^{n} ; \mu\right)$ as the concrete vector fields on $\left(\mathbb{R}^{n}, \mathrm{~d}_{\text {Eucl }}, \mu\right)$. Given any $f \in C_{c}^{\infty}\left(\mathbb{R}^{n}\right)$, we denote by $\nabla f \in L^{2}\left(\mathbb{R}^{n}, \mathbb{R}^{n} ; \mu\right)$ the (equivalence class of the) 'strong' gradient of $f$, i.e. for any $x \in \mathbb{R}^{n}$ we characterise $\nabla f(x) \in \mathbb{R}^{n}$ as the unique vector satisfying

$$
\lim _{y \rightarrow x} \frac{f(y)-f(x)-\nabla f(x) \cdot(y-x)}{|y-x|}=0 .
$$

The Hilbert module $L^{2}\left(\mathbb{R}^{n},\left(\mathbb{R}^{n}\right)^{*} ; \mu\right)$ is the dual module of $L^{2}\left(\mathbb{R}^{n}, \mathbb{R}^{n} ; \mu\right)$, and its elements are said to be the concrete 1-forms on $\left(\mathbb{R}^{n}, \mathrm{~d}_{\text {Eucl }}, \mu\right)$. The 'strong' differential of a given function $f \in C_{c}^{\infty}\left(\mathbb{R}^{n}\right)$ will be denoted by $\mathrm{d} f \in L^{2}\left(\mathbb{R}^{n},\left(\mathbb{R}^{n}\right)^{*} ; \mu\right)$.

The relation between abstract and concrete vector fields on the weighted Euclidean space has been investigated in [27], where the following results have been proven:

Theorem 2.15 (Density in energy of smooth functions) Let $\mu$ be a non-negative Radon measure on $\mathbb{R}^{n}$. Let $f \in W^{1,2}\left(\mathbb{R}^{n}, \mu\right)$ be given. Then, there exists a sequence $\left(f_{i}\right)_{i} \subseteq C_{c}^{\infty}\left(\mathbb{R}^{n}\right)$ such that $f_{i} \rightarrow f$ and $\left|\nabla f_{i}\right| \rightarrow\left|D_{\mu} f\right|$ in $L^{2}(\mu)$.

The proof of the above result was obtained by combining a standard convolution argument with (a stronger variant of) Theorem 2.5. As a consequence, the following statement holds:

Theorem 2.16 (The isometric embedding $\iota_{\mu}$ ) Let $\mu \geq 0$ be a Radon measure on $\mathbb{R}^{n}$. Then, there exists a unique morphism $\mathrm{P}_{\mu}: L^{2}\left(\mathbb{R}^{n},\left(\mathbb{R}^{n}\right)^{*} ; \mu\right) \rightarrow L_{\mu}^{2}\left(T^{*} \mathbb{R}^{n}\right)$ such that

$$
\mathrm{P}_{\mu}(\mathrm{d} f)=\mathrm{d}_{\mu} f, \quad \text { for every } f \in C_{c}^{\infty}\left(\mathbb{R}^{n}\right) .
$$

Calling $\iota_{\mu}: L_{\mu}^{2}\left(T \mathbb{R}^{n}\right) \rightarrow L^{2}\left(\mathbb{R}^{n}, \mathbb{R}^{n} ; \mu\right)$ the adjoint of $\mathrm{P}_{\mu}$, i.e. the unique morphism satisfying

$$
\mathrm{P}_{\mu}(\underline{\omega})(v)=\underline{\omega}\left(l_{\mu}(v)\right), \quad \text { for every } v \in L_{\mu}^{2}\left(T \mathbb{R}^{n}\right) \text { and } \underline{\omega} \in L^{2}\left(\mathbb{R}^{n},\left(\mathbb{R}^{n}\right)^{*} ; \mu\right),
$$

we have that $\left|l_{\mu}(v)\right|=|v|$ holds $\mu$-a.e. on $\mathbb{R}^{n}$ for any given $v \in L_{\mu}^{2}\left(T \mathbb{R}^{n}\right)$.

Remark 2.17 Given any Radon measure $\mu \geq 0$ on $\mathbb{R}^{n}$ and any vector field $v \in L_{\mu}^{2}\left(T \mathbb{R}^{n}\right)$, it holds that $l_{\mu}(v)$ can be characterised as the unique element of $L^{2}\left(\mathbb{R}^{n}, \mathbb{R}^{n} ; \mu\right)$ such that

$$
\int \mathrm{d}_{\mu} f(v) \mathrm{d} \mu=\int \mathrm{d} f\left(l_{\mu}(v)\right) \mathrm{d} \mu, \quad \text { for every } f \in C_{c}^{\infty}\left(\mathbb{R}^{n}\right) .
$$

This readily follows from the fact that $\left\{\mathrm{d} f: f \in C_{c}^{\infty}\left(\mathbb{R}^{n}\right)\right\}$ generates $L^{2}\left(\mathbb{R}^{n},\left(\mathbb{R}^{n}\right)^{*} ; \mu\right)$ and that $l_{\mu}: L_{\mu}^{2}\left(T \mathbb{R}^{n}\right) \rightarrow L^{2}\left(\mathbb{R}^{n}, \mathbb{R}^{n} ; \mu\right)$ is a morphism of $L^{2}(\mu)$-normed $L^{\infty}(\mu)$-modules.

As it was observed in [27], it immediately follows from Theorem 2.16 that Euclidean spaces are universally infinitesimally Hilbertian, in the following sense.

Theorem 2.18 (Infinitesimal Hilbertianity of weighted $\mathbb{R}^{n}$ ) Let $\mu \geq 0$ be a Radon measure on $\mathbb{R}^{n}$. Then, the metric measure space $\left(\mathbb{R}^{d}, \mathrm{~d}_{\mathrm{Eucl}}, \mu\right)$ is infinitesimally Hilbertian. 
We point out that other two different proofs of Theorem 2.18 are known: it directly follows from [18, Theorem 1.1], and it is one of the main achievements of [19]; in Section 3.1, we will briefly describe the strategy of the latter approach. Let us now recall an important consequence of Theorems 2.15 and 2.18. For the reader's usefulness, we also provide its proof.

Corollary 2.19 (Strong density of smooth functions) Let $\mu \geq 0$ be a Radon measure on $\mathbb{R}^{n}$. Let $f \in W^{1,2}\left(\mathbb{R}^{n}, \mu\right)$ be given. Then, there exists a sequence $\left(f_{i}\right)_{i} \subseteq C_{c}^{\infty}\left(\mathbb{R}^{n}\right)$ such that

$$
f_{i} \longrightarrow f, \quad \text { strongly in } L^{2}(\mu),
$$

$$
\left|\nabla f_{i}\right| \longrightarrow\left|D_{\mu} f\right|, \quad \text { strongly in } L^{2}(\mu)
$$

$$
\nabla_{\mu} f_{i} \longrightarrow \nabla_{\mu} f, \quad \text { strongly in } L_{\mu}^{2}\left(T \mathbb{R}^{n}\right)
$$

Proof Thanks to Theorem 2.15, we can find a sequence $\left(f_{i}\right)_{i} \subseteq C_{c}^{\infty}\left(\mathbb{R}^{n}\right)$ satisfying (2.23a) and (2.23b). Given that $\left|\nabla_{\mu} f_{i}\right|=\left|D_{\mu} f_{i}\right| \leq\left|\nabla f_{i}\right|$ holds $\mu$-a.e. for every $i \in \mathbb{N}$, we deduce that the sequence $\left(\nabla_{\mu} f_{i}\right)_{i}$ is bounded in $L_{\mu}^{2}\left(T \mathbb{R}^{n}\right)$. Being $L_{\mu}^{2}\left(T \mathbb{R}^{n}\right)$ Hilbert, there exists $v \in L_{\mu}^{2}\left(T \mathbb{R}^{n}\right)$ such that (up to a not relabelled subsequence) it holds that $\nabla_{\mu} f_{i} \rightarrow v$ weakly in $L_{\mu}^{2}\left(T \mathbb{R}^{n}\right)$. By using Proposition 2.11 (and the Riesz isomorphism), we obtain that $v=\nabla_{\mu} f$. Moreover,

$$
\begin{aligned}
& \int\left|D_{\mu} f\right|^{2} \mathrm{~d} \mu \leq \lim _{i \rightarrow \infty} \int\left|D_{\mu} f_{i}\right|^{2} \mathrm{~d} \mu \\
& \leq \varlimsup_{i \rightarrow \infty} \int\left|D_{\mu} f_{i}\right|^{2} \mathrm{~d} \mu \leq \lim _{i \rightarrow \infty} \int\left|\nabla f_{i}\right|^{2} \mathrm{~d} \mu \stackrel{(1.23 \mathrm{~b})}{=} \int\left|D_{\mu} f\right|^{2} \mathrm{~d} \mu,
\end{aligned}
$$

where the first inequality is granted by the weak convergence $\nabla_{\mu} f_{i} \rightarrow \nabla_{\mu} f$. Consequently, we conclude that $\int\left|D_{\mu} f\right|^{2} \mathrm{~d} \mu=\lim _{i} \int\left|D_{\mu} f_{i}\right|^{2} \mathrm{~d} \mu$ and thus $\nabla_{\mu} f_{i} \rightarrow \nabla_{\mu} f$ strongly in $L_{\mu}^{2}\left(T \mathbb{R}^{n}\right)$, which shows the validity of $(2.23 \mathrm{c})$.

\subsubsection{Divergence of concrete vector fields}

Let $\mu \geq 0$ be a Radon measure on $\mathbb{R}^{n}$. Then, we denote by $D\left(\underline{\operatorname{div}}_{\mu}\right)$ the space of all those vector fields $\underline{v} \in L^{2}\left(\mathbb{R}^{n}, \mathbb{R}^{n} ; \mu\right)$ whose distributional divergence belongs to $L^{2}(\mu)$. Namely, there exists a function $\underline{\operatorname{div}}_{\mu}(\underline{v}) \in L^{2}(\mu)$ such that

$$
\int \nabla f \cdot \underline{v} \mathrm{~d} \mu=-\int f \underline{\operatorname{div}}_{\mu}(\underline{v}) \mathrm{d} \mu, \quad \text { for every } f \in C_{c}^{\infty}\left(\mathbb{R}^{n}\right) .
$$

Observe that $\underline{\operatorname{div}}_{\mu}$ satisfies the Leibniz rule, i.e. it holds that $f \underline{v} \in D\left(\underline{\operatorname{div}}_{\mu}\right)$ and

$$
\underline{\operatorname{div}}_{\mu}(f \underline{v})=f \underline{\operatorname{div}}_{\mu}(\underline{v})+\nabla f \cdot \underline{v}
$$

for every $f \in C_{c}^{\infty}\left(\mathbb{R}^{n}\right)$ and $\underline{v} \in D\left(\underline{\operatorname{div}}_{\mu}\right)$.

Lemma 2.20 (Relation between $\operatorname{div}_{\mu}$ and $\underline{\operatorname{div}}_{\mu}$ ) Let $\mu \geq 0$ be a Radon measure on $\mathbb{R}^{n}$. Then, for any vector field $v \in L_{\mu}^{2}\left(T \mathbb{R}^{n}\right)$ we have that 


$$
v \in D\left(\operatorname{div}_{\mu}\right) \quad \Longleftrightarrow \quad l_{\mu}(v) \in D\left(\underline{\operatorname{div}}_{\mu}\right) .
$$

In this case, it holds that $\operatorname{div}_{\mu}(v)=\underline{\operatorname{div}}_{\mu}\left(l_{\mu}(v)\right)$ in the $\mu$-a.e. sense.

Proof On the one hand, suppose $v \in D\left(\operatorname{div}_{\mu}\right)$. Then, for any $f \in C_{c}^{\infty}\left(\mathbb{R}^{n}\right)$ it holds that

$$
\begin{aligned}
\int \nabla f \cdot l_{\mu}(v) \mathrm{d} \mu & =\int \mathrm{d} f\left(\iota_{\mu}(v)\right) \mathrm{d} \mu \stackrel{(1.22)}{=} \int \mathrm{d}_{\mu} f(v) \mathrm{d} \mu=\int\left\langle\nabla_{\mu} f, v\right\rangle \mathrm{d} \mu \\
& =-\int f \operatorname{div}_{\mu}(v) \mathrm{d} \mu,
\end{aligned}
$$

whence $\imath_{\mu}(v) \in D\left(\underline{\operatorname{div}}_{\mu}\right)$ and $\underline{\operatorname{div}}_{\mu}\left(l_{\mu}(v)\right)=\operatorname{div}_{\mu}(v)$ holds $\mu$-a.e. on $\mathbb{R}^{n}$. On the other hand, suppose $\imath_{\mu}(v) \in D\left({\underline{\overline{\operatorname{div}}_{\mu}}}_{\mu}\right)$. Let us fix any function $f \in W^{1,2}\left(\mathbb{R}^{n}, \mu\right)$. Corollary 2.19 grants the existence of $\left(f_{i}\right)_{i} \subseteq{\overline{C_{c}^{\infty}}}^{\mu}\left(\mathbb{R}^{n}\right)$ such that $f_{i} \rightarrow f$ in $L^{2}(\mu)$ and $\nabla_{\mu} f_{i} \rightarrow \nabla_{\mu} f$ in $L_{\mu}^{2}\left(T \mathbb{R}^{n}\right)$. Therefore, it holds that

$$
\begin{aligned}
\int\left\langle\nabla_{\mu} f, v\right\rangle \mathrm{d} \mu & =\lim _{i \rightarrow \infty} \int\left\langle\nabla_{\mu} f_{i}, v\right\rangle \mathrm{d} \mu \stackrel{(1.22)}{=} \lim _{i \rightarrow \infty} \int \nabla f_{i} \cdot l_{\mu}(v) \mathrm{d} \mu \\
& =-\lim _{i \rightarrow \infty} \int f_{i} \underline{\operatorname{div}}_{\mu}\left(l_{\mu}(v)\right) \mathrm{d} \mu \\
& =-\int f \underline{\operatorname{div}}_{\mu}\left(l_{\mu}(v)\right) \mathrm{d} \mu .
\end{aligned}
$$

This shows that $v \in D\left(\operatorname{div}_{\mu}\right)$ and $\operatorname{div}_{\mu}(v)=\underline{\operatorname{div}}_{\mu}\left(l_{\mu}(v)\right)$ holds $\mu$-a.e. on $\mathbb{R}^{n}$, as required.

\subsection{Distributions on the Euclidean space}

We denote by $\operatorname{Gr}\left(\mathbb{R}^{n}\right)$ the Grassmannian of $\mathbb{R}^{n}$, i.e. the family of all linear subspaces of $\mathbb{R}^{n}$. We endow $\operatorname{Gr}\left(\mathbb{R}^{n}\right)$ with the distance

$$
\begin{aligned}
& \mathrm{d}_{\mathrm{Gr}\left(\mathbb{R}^{n}\right)}(V, W):=\max \left\{\sup _{v \in V,} \inf _{w \in W,}|v-w|, \sup _{w \in W,} \inf _{v \in V,}|v-w|\right\}, \\
& |v| \leq 1 \quad|w| \leq 1 \quad|w| \leq 1 \quad|v| \leq 1
\end{aligned}
$$

for every $V, W \in \operatorname{Gr}\left(\mathbb{R}^{n}\right)$,

i.e. $\mathrm{d}_{\operatorname{Gr}\left(\mathbb{R}^{n}\right)}(V, W)$ is the Hausdorff distance in $\mathbb{R}^{n}$ between the closed unit balls of $V$ and $W$. It holds that $\left(\operatorname{Gr}\left(\mathbb{R}^{n}\right), \mathrm{d}_{\mathrm{Gr}\left(\mathbb{R}^{n}\right)}\right)$ is a compact metric space; see, for instance, [2].

Definition 2.21 (Distribution) A distribution on $\mathbb{R}^{n}$ is a Borel map $V: \mathbb{R}^{n} \rightarrow \operatorname{Gr}\left(\mathbb{R}^{n}\right)$. Given any Radon measure $\mu$ on $\mathbb{R}^{n}$, we denote by $\mathscr{D}_{n}(\mu)$ the family of all distributions on $\mathbb{R}^{n}$, considered up to $\mu$-a.e. equality. Given any $V \in \mathscr{D}_{n}(\mu)$, we define $\Gamma(V) \subseteq L^{2}\left(\mathbb{R}^{n}, \mathbb{R}^{n} ; \mu\right)$ as

$$
\Gamma(V):=\left\{\underline{v} \in L^{2}\left(\mathbb{R}^{n}, \mathbb{R}^{n} ; \mu\right) \mid \underline{v}(x) \in V(x) \text {, for } \mu \text {-a.e. } x \in \mathbb{R}^{n}\right\} .
$$

Moreover, we define a partial order on $\mathscr{D}_{n}(\mu)$ in the following way: given any $V, W \in \mathscr{D}_{n}(\mu)$, we declare that $V \leq W$ provided it holds that $V(x) \subseteq W(x)$ for $\mu$-a.e. $x \in \mathbb{R}^{n}$. 
It can be readily checked that $\Gamma(V)$ is an $L^{2}(\mu)$-normed $L^{\infty}(\mu)$-submodule of $L^{2}\left(\mathbb{R}^{n}, \mathbb{R}^{n} ; \mu\right)$.

Proposition 2.22 Let $\mu \geq 0$ be a Radon measure on $\mathbb{R}^{n}$. Then, the mapping $V \mapsto \Gamma(V)$ is a bijection between $\mathscr{D}_{n}(\mu)$ and the family of $L^{2}(\mu)$-normed $L^{\infty}(\mu)$-submodules of $L^{2}\left(\mathbb{R}^{n}, \mathbb{R}^{n} ; \mu\right)$. Moreover, the map $\Gamma$ is order-preserving, i.e. one has $V \leq W$ if and only if $\Gamma(V) \subseteq \Gamma(W)$.

Proof The only non-trivial fact to check is that the mapping $\Gamma$ is surjective. To this aim, let us fix an $L^{2}(\mu)$-normed $L^{\infty}(\mu)$-submodule $\mathscr{M}$ of $L^{2}\left(\mathbb{R}^{n}, \mathbb{R}^{n} ; \mu\right)$. Also, take any countable, dense $\mathbb{Q}$-linear subspace $\left(\underline{v}_{i}\right)_{i}$ of $\mathscr{M}$. Define

$$
V(x):=\operatorname{cl}\left\{\underline{v}_{i}(x) \mid i \in \mathbb{N}\right\} \in \operatorname{Gr}\left(\mathbb{R}^{n}\right), \quad \text { for } \mu \text {-a.e. } x \in \mathbb{R}^{n} .
$$

The resulting map $V: \mathbb{R}^{n} \rightarrow \operatorname{Gr}\left(\mathbb{R}^{n}\right)$ is Borel. Indeed, for every $W \in \operatorname{Gr}\left(\mathbb{R}^{n}\right)$ we have that

$$
\begin{aligned}
& \mathrm{d}_{\mathrm{Gr}\left(\mathbb{R}^{n}\right)}(V(x), W) \\
& \quad=\max \left\{\sup _{i \in \mathbb{N}} \inf _{j \in \mathbb{N}}\left|\frac{\underline{v}_{i}(x)}{\max \left\{\left|\underline{v}_{i}(x)\right|, 1\right\}}-w_{j}\right|, \sup _{j \in \mathbb{N}} \inf _{i \in \mathbb{N}}\left|\frac{\underline{v}_{i}(x)}{\max \left\{\left|\underline{v}_{i}(x)\right|, 1\right\}}-w_{j}\right|\right\}
\end{aligned}
$$

holds for $\mu$-a.e. $x \in \mathbb{R}^{n}$, where $\left(w_{j}\right)_{j}$ is any dense sequence in the closed unit ball of $W$, thus accordingly $x \mapsto \mathrm{d}_{\operatorname{Gr}\left(\mathbb{R}^{n}\right)}(V(x), W)$ is $\mu$-a.e. equivalent to a Borel function. Then, $V \in \mathscr{D}_{n}(\mu)$.

Let us now prove that $\mathscr{M}=\Gamma(V)$. Given that $\underline{v}_{i} \in \Gamma(V)$ for every $i \in \mathbb{N}$ by construction and $\mathscr{M}=\operatorname{cl}\left\{\underline{v}_{i}: i \in \mathbb{N}\right\}$, we deduce that $\mathscr{M} \subseteq \underline{\Gamma}(V)$. Conversely, fix any $\underline{v} \in \Gamma(V)$. By dominated convergence theorem we see that the sequence $\left(\underline{w}_{j}\right)_{j} \subseteq \Gamma(\bar{V})$, given by $\underline{w}_{j}:=\mathbb{1}_{B_{j}(0)} \underline{v}$ for all $j \in \mathbb{N}$, converges to $\underline{v}$ in $\Gamma(V)$. Fix $j_{0} \in \mathbb{N}$ satisfying $\mu\left(B_{j_{0}}(0)\right)>0$. Given any $j \geq j_{0}$, we infer from (2.26) that there is a Borel partition $\left(E_{i}^{j}\right)_{i}$ of $B_{j}(0)$ having the property that

$$
\left|\underline{v}_{i}(x)-\underline{v}(x)\right|^{2} \leq \frac{1}{j^{2} \mu\left(B_{j}(0)\right)}, \quad \text { for every } i \in \mathbb{N} \text { and } \mu \text {-a.e. } x \in E_{i}^{j} .
$$

Define $\underline{z}_{j}:=\sum_{i=1}^{\infty} \mathbb{1}_{E_{i}^{j}} \underline{v}_{i} \in \mathscr{M}$. By exploiting the inequality in (2.27), we thus obtain that

$$
\int\left|\underline{z}_{j}-\underline{w}_{j}\right|^{2} \mathrm{~d} \mu=\sum_{i=1}^{\infty} \int_{E_{i}^{j}} \underline{v}_{i}-\left.\underline{v}\right|^{2} \mathrm{~d} \mu \leq \sum_{i=1}^{\infty} \frac{\mu\left(E_{i}^{j}\right)}{j^{2} \mu\left(B_{j}(0)\right)}=\frac{1}{j^{2}} .
$$

Therefore, we conclude that $\left\|\underline{z}_{j}-\underline{v}\right\|_{\Gamma(V)} \leq \frac{1}{j}+\left\|\underline{w}_{j}-\underline{v}\right\|_{\Gamma(V)} \rightarrow 0$, so that $\underline{v} \in \mathscr{M}$.

Remark 2.23 The statement of Proposition 2.22 is a particular instance of a more general result proven in [34], concerning the representation of a certain class of normed modules as spaces of sections of a measurable Banach bundle. Nevertheless, in the special case under consideration (i.e. only submodules of $L^{2}\left(\mathbb{R}^{n}, \mathbb{R}^{n} ; \mu\right)$ are taken into account) the argument is simpler than the original one in [34], so we opted for providing an easier proof.

Lemma 2.24 Let $\mu$ be a Radon measure on $\mathbb{R}^{n}$. Let $\mathscr{V}$ be a linear subspace of $L^{2}\left(\mathbb{R}^{n}, \mathbb{R}^{n} ; \mu\right)$ such that $g \underline{v} \in \mathscr{V}$ holds for every $g \in C_{c}^{\infty}\left(\mathbb{R}^{n}\right)$ and $\underline{v} \in \mathscr{V}$. Given a dense sequence $\left(\underline{v}_{i}\right)_{i} \subseteq \mathscr{V}$, we define $V(x):=\operatorname{cl}\left\{\underline{v}_{i}(x): i \in \mathbb{N}\right\}$ for $\mu$-a.e. $x \in \mathbb{R}^{n}$. Then 
$\{V(x)\}_{x \in \mathbb{R}^{n}}$ is a family of linear subspaces of $\mathbb{R}^{n}$, which are $\mu$-a.e. independent of $\left(\underline{v}_{i}\right)_{i}$. Moreover, it holds that

$$
\operatorname{cl} \mathscr{V}=\left\{\underline{v} \in L^{2}\left(\mathbb{R}^{n}, \mathbb{R}^{n} ; \mu\right) \mid \underline{v}(x) \in V(x), \text { for } \mu \text {-a.e. } x \in \mathbb{R}^{n}\right\} .
$$

In particular, the map $V: \mathbb{R}^{n} \rightarrow \operatorname{Gr}\left(\mathbb{R}^{n}\right)$ is a distribution on $\mathbb{R}^{n}$, the Banach space $\operatorname{cl} \mathscr{V}$ is an $L^{2}(\mu)$-normed $L^{\infty}(\mu)$-submodule of $L^{2}\left(\mathbb{R}^{n}, \mathbb{R}^{n} ; \mu\right)$, and $\Gamma(V)=\operatorname{cl} \mathscr{V}$.

Proof The first part of the statement follows, e.g. from [12, Lemma A.1]. The fact that $V$ is a distribution on $\mathbb{R}^{n}$ can be proved by arguing exactly as in the proof of Proposition 2.22, whence the remaining claims immediately follow.

Remark 2.25 (Orthogonal projection) Let $\mu \geq 0$ be a Radon measure on $\mathbb{R}^{n}$. Let $V \in \mathscr{D}_{n}(\mu)$ be given. We define the orthogonal projection mapping $\operatorname{pr}_{V}: L^{2}\left(\mathbb{R}^{n}, \mathbb{R}^{n} ; \mu\right) \rightarrow \Gamma(V)$ as

$$
\operatorname{pr}_{V}(\underline{v})(x):=\pi_{V(x)}(\underline{v}(x)), \quad \text { for } \mu \text {-a.e. } x \in \mathbb{R}^{n},
$$

where $\pi_{V(x)}: \mathbb{R}^{n} \rightarrow V(x)$ is the standard orthogonal projection. Clearly, the mapping $\operatorname{pr}_{V}$ is a surjective, 1-Lipschitz morphism of $L^{2}(\mu)$-normed $L^{\infty}(\mu)$-modules.

Remark 2.26 (Orthogonal complement, II) Given any Radon measure $\mu$ on $\mathbb{R}^{n}$ and any distribution $V \in \mathscr{D}_{n}(\mu)$, we define the orthogonal complement $V^{\perp} \in \mathscr{D}_{n}(\mu)$ of $V$ as

$$
V^{\perp}(x):=(V(x))^{\perp} \subseteq \mathbb{R}^{n}, \quad \text { for } \mu \text {-a.e. } x \in \mathbb{R}^{n} .
$$

Moreover, observe that $\Gamma\left(V^{\perp}\right)=\Gamma(V)^{\perp}$, where $\Gamma(V)^{\perp}$ is defined as in Remark 2.7.

\section{Characterisation of the Sobolev space on weighted Euclidean spaces}

\subsection{Alberti-Marchese distribution}

In our investigation of the Sobolev space associated with a weighted Euclidean space, a key role is played by the following result, whose statement can be roughly summed up in this way: given a Radon measure $\mu$ on $\mathbb{R}^{n}$, there is a 'maximal' distribution $V_{\mu}$ on $\mathbb{R}^{n}$ along which all Lipschitz functions are $\mu$-a.e. (Fréchet) differentiable.

Theorem 3.1 (Alberti-Marchese distribution [1]) Let $\mu \geq 0$ be a Radon measure on $\mathbb{R}^{n}$. Then, there exists a unique distribution $V_{\mu} \in \mathscr{D}_{n}(\mu)$ such that the following properties hold:

(i) Every function $f \in \operatorname{LIP}_{c}\left(\mathbb{R}^{n}\right)$ is $\mu$-a.e. differentiable with respect to $V_{\mu}$, i.e. there exists a vector field $\nabla_{\mathrm{AM}} f \in \Gamma\left(V_{\mu}\right)$ such that

$$
\lim _{V_{\mu}(x) \ni v \rightarrow 0} \frac{f(x+v)-f(x)-\nabla_{\mathrm{AM}} f(x) \cdot v}{|v|}=0, \quad \text { for } \mu \text {-a.e. } x \in \mathbb{R}^{n} .
$$

(ii) There exists a function $f_{0} \in \operatorname{LIP}\left(\mathbb{R}^{n}\right)$ such that for $\mu$-a.e. $x \in \mathbb{R}^{n}$ it holds that $f_{0}$ is not differentiable at $x$ with respect to any direction $v \in \mathbb{R}^{n} \backslash V_{\mu}(x)$. 
We call $V_{\mu}$ the Alberti-Marchese distribution associated with $\mu$.

In [1], the object $V_{\mu}$ is called the 'decomposability bundle' of $\mu$. Here, we chose the term 'distribution' in order to be consistent with our Definition 2.21. Moreover, Theorem 3.1 was actually proven under the additional assumption of $\mu$ being a finite measure, whence the case of a possibly infinite Radon measure follows by arguing as in [19, Remark 1.6].

Remark 3.2 It follows from Rademacher theorem that

$$
V_{\mathcal{L}^{n}}(x)=\mathbb{R}^{n}, \quad \text { for } \mathcal{L}^{n} \text {-a.e. } x \in \mathbb{R}^{n} .
$$

In particular, if $\mu \ll \mathcal{L}^{n}$, then $V_{\mu}(x)=\mathbb{R}^{n}$ holds for $\mu$-a.e. $x \in \mathbb{R}^{n}$.

We shall refer to $\nabla_{\mathrm{AM}}$ as the Alberti-Marchese gradient operator. It readily follows from (3.1) that the element $\nabla_{\mathrm{AN}} f$ is uniquely determined (up to $\mu$-a.e. equality). Moreover,

$$
\begin{array}{ll}
\nabla_{\mathrm{AM}}(f+g)(x)=\nabla_{\mathrm{AM}} f(x)+\nabla_{\mathrm{AM}} g(x), & \text { for } \mu \text {-a.e. } x \in \mathbb{R}^{n}, \\
\nabla_{\mathrm{AM}}(f-g)(x)=\nabla_{\mathrm{AM}} f(x)-\nabla_{\mathrm{AM}} g(x), & \text { for } \mu \text {-a.e. } x \in \mathbb{R}^{n},
\end{array}
$$

are satisfied for every $f, g \in \operatorname{LIP}_{c}\left(\mathbb{R}^{n}\right)$. Let us also recall that it holds that

$$
\left|\nabla_{\mathrm{AM}} f(x)\right| \leq \operatorname{lip}(f)(x), \quad \text { for } \mu \text {-a.e. } x \in \mathbb{R}^{n},
$$

as shown in [19, Remark 1.7].

\subsubsection{Consequences of Alberti-Marchese theorem}

Aim of this section is to illustrate the relation between the Alberti-Marchese distribution and the Sobolev space on weighted $\mathbb{R}^{n}$, investigated in [19]. We collect in the following statement the main results of [19, Section 2].

Theorem 3.3 Let $\mu \geq 0$ be a Radon measure on $\mathbb{R}^{n}$. Then, the following properties hold:

(i) Let $\pi$ be a test plan on $\left(\mathbb{R}^{n}, \mathrm{~d}_{\mathrm{Eucl}}, \mu\right)$. Then, for $\pi$-a.e. curve $\gamma$ it holds that

$$
\dot{\gamma}_{t} \in V_{\mu}\left(\gamma_{t}\right), \quad \text { for } \mathcal{L}_{1} \text {-a.e. } t \in[0,1] .
$$

(ii) Let $f \in \operatorname{LIP}_{c}\left(\mathbb{R}^{n}\right)$ be given. Then $\left|\nabla_{\mathrm{AM}} f\right| \in L^{2}(\mu)$ is a weak upper gradient of $f$.

(iii) Let $f \in W^{1,2}\left(\mathbb{R}^{n}, \mu\right)$ be given. Then, there exists a sequence $\left(f_{i}\right)_{i} \subseteq \operatorname{LIP}_{c}\left(\mathbb{R}^{n}\right)$ such that $f_{i} \rightarrow f$ and $\left|\nabla_{\mathrm{AN}} f_{i}\right| \rightarrow\left|D_{\mu} f\right|$ in the strong topology of $L^{2}(\mu)$.

As we already mentioned in the paragraph below Theorem 2.18 , the universal infinitesimal Hilbertianity of $\mathbb{R}^{n}$ was obtained in [19, Theorem 2.3] as a consequence of Theorem 3.3. The argument was the following: the Cheeger energy $E_{\mathrm{Ch}}$ is the lower semicontinuous envelope of the Alberti-Marchese energy functional $E_{\mathrm{AM}}: L^{2}(\mu) \rightarrow[0,+\infty]$, given by

$$
E_{\mathrm{AM}}(f):= \begin{cases}\frac{1}{2} \int\left|\nabla_{\mathrm{AM}} f\right|^{2} \mathrm{~d} \mu, & \text { if } f \in \operatorname{LIP}_{c}\left(\mathbb{R}^{n}\right), \\ +\infty, & \text { otherwise, }\end{cases}
$$


which is clearly 2-homogeneous by construction, and satisfies the parallelogram rule by (3.2). Consequently, the Cheeger energy associated with $\left(\mathbb{R}^{n}, d_{\text {Eucl }}, \mu\right)$ satisfies the parallelogram rule, thus yielding the sought conclusion.

\subsection{Identification of the tangent module}

Let $\mu$ be a given Radon measure on $\mathbb{R}^{n}$. We know from Theorem 2.16 that the tangent module $L_{\mu}^{2}\left(T \mathbb{R}^{n}\right)$ can be canonically seen as a submodule of $L^{2}\left(\mathbb{R}^{n}, \mathbb{R}^{n} ; \mu\right)$, whence (by Proposition 2.22) we have a natural notion of tangent distribution $T_{\mu}$. In this section, we provide some alternative characterisations of $T_{\mu}$, thus showing (as described in the introduction) that our approach is equivalent to the ones introduced in [9] and [40, 41]. Some of the proofs that we will carry out are inspired by [33].

\subsubsection{Tangent distribution}

We introduce the notion of tangent distribution on $\left(\mathbb{R}^{n}, \mathrm{~d}_{\mathrm{Eucl}}, \mu\right)$ :

Definition 3.4 (Tangent distribution) Let $\mu$ be a Radon measure on $\mathbb{R}^{n}$. Then, we define the tangent distribution $T_{\mu}$ as the unique element of $\mathscr{D}_{n}(\mu)$ such that

$$
\Gamma\left(T_{\mu}\right)=\iota_{\mu}\left(L_{\mu}^{2}\left(T \mathbb{R}^{n}\right)\right),
$$

where $\quad l_{\mu}: L_{\mu}^{2}\left(T \mathbb{R}^{n}\right) \rightarrow L^{2}\left(\mathbb{R}^{n}, \mathbb{R}^{n} ; \mu\right)$ is the isometric embedding described in Theorem 2.16.

Remark 3.5 It is straightforward to check that the module $L_{\mu}^{2}\left(T \mathbb{R}^{n}\right)$ has dimension $k$ on a given Borel set $E \subseteq \mathbb{R}^{n}$ with $\mu(E)>0$ if and only if $\operatorname{dim} T_{\mu}(x)=k$ for $\mu$-a.e. $x \in E$.

The following result shows that 'test plans are tangent to the distribution $T_{\mu}$, , in a sense.

Lemma 3.6 Let $\mu$ be a Radon measure on $\mathbb{R}^{n}$. Let $\pi$ be a given test plan on $\left(\mathbb{R}^{n}, \mathrm{~d}_{\mathrm{Eucl}}, \mu\right)$. Then, for $\pi$-a.e. curve $\gamma$ it holds that

$$
\dot{\gamma}_{t} \in T_{\mu}\left(\gamma_{t}\right), \quad \text { for } \mathcal{L}_{1} \text {-a.e. } t \in[0,1] .
$$

Proof Let $\pi$ be a given test plan on $\left(\mathbb{R}^{n}, \mathrm{~d}_{\mathrm{Eucl}}, \mu\right)$. Given any $t \in[0,1]$, consider the pullback morphisms $\mathrm{e}_{t}^{*} \mathrm{P}_{\mu}: \mathrm{e}_{t}^{*} L^{2}\left(\mathbb{R}^{n},\left(\mathbb{R}^{n}\right)^{*} ; \mu\right) \rightarrow \mathrm{e}_{t}^{*} L_{\mu}^{2}\left(T^{*} \mathbb{R}^{n}\right)$ and $\mathrm{e}_{t}^{*} l_{\mu}: \mathrm{e}_{t}^{*} L_{\mu}^{2}\left(T \mathbb{R}^{n}\right) \rightarrow \mathrm{e}_{t}^{*} L^{2}\left(\mathbb{R}^{n}, \mathbb{R}^{n} ; \mu\right)$ as in Theorem 2.9. The spaces $\mathrm{e}_{t}^{*} L_{\mu}^{2}\left(T \mathbb{R}^{n}\right)$ and $\mathrm{e}_{t}^{*} L^{2}\left(\mathbb{R}^{n}, \mathbb{R}^{n} ; \mu\right)$ can be identified with the dual modules of $\mathrm{e}_{t}^{*} L_{\mu}^{2}\left(T^{*} \mathbb{R}^{n}\right)$ and $\mathrm{e}_{t}^{*} L^{2}\left(\mathbb{R}^{n},\left(\mathbb{R}^{n}\right)^{*} ; \mu\right)$, respectively, as a consequence of the separability of $L^{2}\left(\mathbb{R}^{n}, \mathbb{R}^{n} ; \mu\right)$ (which can be readily checked) and of its subspace $\iota_{\mu}\left(L_{\mu}^{2}\left(T \mathbb{R}^{n}\right)\right)$; cf. [24, Theorem 1.6.7]. Since $l_{\mu}$ is the adjoint of $\mathrm{P}_{\mu}$, it holds that $\mathrm{e}_{t}^{*} l_{\mu}$ is the adjoint of $\mathrm{e}_{t}^{*} \mathrm{P}_{\mu}$, thus in particular for any element $z \in \mathrm{e}_{t}^{*} L_{\mu}^{2}\left(T \mathbb{R}^{n}\right)$ we have that

$$
\left(\left(\mathrm{e}_{t}^{*} \mathrm{P}_{\mu}\right)\left(\mathrm{e}_{t}^{*} \mathrm{~d} f\right)\right)(z)=\left(\mathrm{e}_{t}^{*} \mathrm{~d} f\right)\left(\left(\mathrm{e}_{t}^{*} \iota_{\mu}\right)(z)\right) \quad \pi \text {-a.e., } \quad \text { for every } f \in C_{c}^{\infty}\left(\mathbb{R}^{n}\right) .
$$

Moreover, the morphism $\mathrm{e}_{t}^{*} \iota_{\mu}$ preserves the pointwise norm. In order to prove it, notice that

$$
\left|\left(\mathrm{e}_{t}^{*} \iota_{\mu}\right)\left(\mathrm{e}_{t}^{*} v\right)\right|=\left|\mathrm{e}_{t}^{*}\left(l_{\mu}(v)\right)\right|=\left|l_{\mu}(v)\right| \circ \mathrm{e}_{t}=|v| \circ \mathrm{e}_{t}=\left|\mathrm{e}_{t}^{*} v\right| \quad \pi \text {-a.e., } \quad \text { for every } v \in L_{\mu}^{2}\left(T \mathbb{R}^{n}\right),
$$


whence $\mathrm{e}_{t}^{*} l_{\mu}$ is an isometry as we know that $\left\{\mathrm{e}_{t}^{*} v: v \in L_{\mu}^{2}\left(T \mathbb{R}^{n}\right)\right\}$ generates $\mathrm{e}_{t}^{*} L_{\mu}^{2}\left(T \mathbb{R}^{n}\right)$.

One can readily check that $\mathrm{e}_{t}^{*} L^{2}\left(\mathbb{R}^{n}, \mathbb{R}^{n} ; \mu\right)$ can be identified with the space $\mathbb{B}_{\pi}$, the pullback map e $\mathrm{e}_{t}^{*}: L^{2}\left(\mathbb{R}^{n}, \mathbb{R}^{n} ; \mu\right) \rightarrow \mathbb{B}_{\pi}$ being given by $\mathrm{e}_{t}^{*} \underline{v}:=\underline{v o e}_{t}$ for every $\underline{v} \in L^{2}\left(\mathbb{R}^{n}, \mathbb{R}^{n} ; \mu\right)$. An analogous statement holds for $\mathrm{e}_{t}^{*} L^{2}\left(\mathbb{R}^{n},\left(\mathbb{R}^{n}\right)^{*} ; \mu\right)$. Observe that

$$
\left(\mathrm{e}_{t}^{*} l_{\mu}\right)\left(\mathrm{e}_{t}^{*} L_{\mu}^{2}\left(T \mathbb{R}^{n}\right)\right)=\left\{\underline{z} \in \mathbb{B}_{\pi} \mid \underline{z}(\gamma) \in T_{\mu}\left(\gamma_{t}\right), \text { for } \pi \text {-a.e. } \gamma\right\} .
$$

Let us consider, for $\mathcal{L}_{1}$-a.e. $t \in[0,1]$, the velocity $\pi_{t}^{\prime} \in \mathrm{e}_{t}^{*} L_{\mu}^{2}\left(T \mathbb{R}^{n}\right)$ of $\pi$ as in Proposition 2.12. We deduce from (2.15) that for any given function $f \in C_{c}^{\infty}\left(\mathbb{R}^{n}\right)$ it holds that

$$
(f \circ \gamma)_{t}^{\prime}=\left(\mathrm{e}_{t}^{*} \mathrm{~d}_{\mu} f\right)\left(\boldsymbol{\pi}_{t}^{\prime}\right)(\gamma), \quad \text { for } \boldsymbol{\pi} \text {-a.e. } \gamma \text {. }
$$

For $\mathcal{L}_{1}$-a.e. $t \in[0,1]$, consider the mapping $\operatorname{Der}_{t} \in \mathbb{B}_{\pi}$ defined in (2.18). We claim that

$$
\left(\mathrm{e}_{t}^{*} \iota_{\mu}\right)\left(\boldsymbol{\pi}_{t}^{\prime}\right)=\operatorname{Der}_{t}, \quad \text { for } \mathcal{L}_{1} \text {-a.e. } t \in[0,1]
$$

Given any function $f \in C_{c}^{\infty}\left(\mathbb{R}^{n}\right)$, we have that for $\pi$-a.e. curve $\gamma$ it holds that

$$
\begin{aligned}
& \left(\left(\mathrm{e}_{t}^{*} \mathrm{P}_{\mu}\right)\left(\mathrm{e}_{t}^{*} \mathrm{~d} f\right)\right)\left(\boldsymbol{\pi}_{t}^{\prime}\right)(\gamma) \\
& \quad=\left(\mathrm{e}_{t}^{*} \mathrm{~d}_{\mu} f\right)\left(\boldsymbol{\pi}_{t}^{\prime}\right)(\gamma) \stackrel{(2.9)}{=}(f \circ \gamma)_{t}^{\prime}=\left(\mathrm{d} f \circ \mathrm{e}_{t}\right)\left(\operatorname{Der}_{t}\right)(\gamma)=\left(\mathrm{e}_{t}^{*} \mathrm{~d} f\right)\left(\operatorname{Der}_{t}\right)(\gamma) .
\end{aligned}
$$

Since the identity in (3.7) actually characterises $\mathrm{e}_{t}^{*} l_{\mu}$, we deduce that the claim (3.10) holds. In particular, we have that $\operatorname{Der}_{t} \in\left(\mathrm{e}_{t}^{*} l_{\mu}\right)\left(\mathrm{e}_{t}^{*} L_{\mu}^{2}\left(T \mathbb{R}^{n}\right)\right)$ for $\mathcal{L}_{1}$-a.e. $t \in[0,1]$, whence $(3.8)$ yields

$$
\dot{\gamma}_{t}=\operatorname{Der}_{t}(\gamma) \in T_{\mu}\left(\gamma_{t}\right), \quad \text { for }\left(\pi \otimes \mathcal{L}_{1}\right) \text {-a.e. }(\gamma, t) .
$$

Thanks to Fubini theorem, we finally conclude that the sought property (3.6) is satisfied.

Clearly, in order to identify the minimal weak upper gradient of a given Sobolev function, it is sufficient to look at the directions that are selected by the test plans. The following result makes this claim precise.

Lemma 3.7 Let $\mu$ be a Radon measure on $\mathbb{R}^{n}$. Let $V \in \mathscr{D}_{n}(\mu)$ satisfy the following property: given any test plan $\pi$ on $\left(\mathbb{R}^{n}, \mathrm{~d}_{\mathrm{Eucl}}, \mu\right)$, it holds that $\dot{\gamma}_{t} \in V\left(\gamma_{t}\right)$ for $\left(\boldsymbol{\pi} \otimes \mathcal{L}_{1}\right)$-a.e. $(\gamma, t)$. Then, for any function $f \in C_{c}^{\infty}\left(\mathbb{R}^{n}\right)$ we have that $\left|\operatorname{pr}_{V}(\nabla f)\right|$ is a weak upper gradient of $f$.

Proof Fix any test plan $\pi$ on $\left(\mathbb{R}^{n}, \mathrm{~d}_{\text {Eucl }}, \mu\right)$. Then for $\pi$-a.e. curve $\gamma$ it holds that

$$
\left|(f \circ \gamma)_{t}^{\prime}\right|=\left|\nabla f\left(\gamma_{t}\right) \cdot \dot{\gamma}_{t}\right|=\left|\operatorname{pr}_{V}(\nabla f)\left(\gamma_{t}\right) \cdot \dot{\gamma}_{t}\right| \leq\left|\operatorname{pr}_{V}(\nabla f)\right|\left(\gamma_{t}\right)\left|\dot{\gamma}_{t}\right|, \quad \text { for } \mathcal{L}_{1} \text {-a.e. } t \in[0,1] .
$$

By arbitrariness of $\pi$, we conclude that $\left|\operatorname{pr}_{V}(\nabla f)\right|$ is a weak upper gradient of $f$.

\subsubsection{An axiomatic notion of weak gradient}

Another possible way to define the tangent fibres is via the vectorial relaxation procedure proposed by Zhikov in [40, 41] and studied by Louet in [33]. Below we introduce a generalisation of such approach, tailored for our purposes. 
Definition 3.8 ( $G$-structure) Let $\mu \geq 0$ be a Radon measure on $\mathbb{R}^{n}$. Then, by $G$-structure on $\left(\mathbb{R}^{n}, \mathrm{~d}_{\text {Eucl }}, \mu\right)$ we mean a couple $(\mathcal{V}, \bar{\nabla})$ satisfying the following list of axioms:

A1. $\mathcal{V}$ is a linear subspace of $W^{1,2}\left(\mathbb{R}^{n}, \mu\right)$ containing $C_{c}^{\infty}\left(\mathbb{R}^{n}\right)$.

A2. $\bar{\nabla}: \mathcal{V} \rightarrow L^{2}\left(\mathbb{R}^{n}, \mathbb{R}^{n} ; \mu\right)$ is a linear operator.

A3. $|\bar{\nabla} f|$ is a weak upper gradient of $f$ for any $f \in \mathcal{V}$, with $|\bar{\nabla} f| \in L^{\infty}(\mu)$ if $f \in C_{c}^{\infty}\left(\mathbb{R}^{n}\right)$.

A4. $\bar{\nabla}$ satisfies the Leibniz rule, i.e. if $f \in \mathcal{V}$ and $g \in C_{c}^{\infty}\left(\mathbb{R}^{n}\right)$, then $f g \in \mathcal{V}$ and

$$
\bar{\nabla}(f g)=f \bar{\nabla} g+g \bar{\nabla} f, \quad \text { in the } \mu \text {-a.e. sense. }
$$

A5. Calling $E_{G}: L^{2}(\mu) \rightarrow[0,+\infty]$ the energy functional

$$
E_{G}(f):= \begin{cases}\frac{1}{2} \int|\bar{\nabla} f|^{2} \mathrm{~d} \mu, & \text { if } f \in \mathcal{V}, \\ +\infty, & \text { otherwise }\end{cases}
$$

it holds that $E_{\mathrm{Ch}}$ is the lower semicontinuous envelope of $E_{G}$.

The term ' $G$-structure' is somehow inspired by the notion of $D$-structure, which has been proposed by V. Gol'dshtein and M. Troyanov in the paper [28]. Therein, they developed an axiomatic theory of Sobolev spaces on general metric measure spaces. In our setting, thanks to the presence of an underlying linear structure, the axiomatisation can be formulated in terms of 'gradients' rather than 'moduli of the gradients'.

Remark 3.9 (Density in energy) Observe that axiom A5 is equivalent to requiring that the elements of $\mathcal{V}$ are dense in energy in $W^{1,2}\left(\mathbb{R}^{n}, \mu\right)$, i.e. for every $f \in W^{1,2}\left(\mathbb{R}^{n}, \mu\right)$ there exists a sequence $\left(f_{i}\right)_{i} \subseteq \mathcal{V}$ such that $f_{i} \rightarrow f$ and $\left|\bar{\nabla} f_{i}\right| \rightarrow\left|D_{\mu} f\right|$ strongly in $L^{2}(\mu)$.

Example 3.10 (Examples of $G$-structures) Let us describe two examples of $G$-structures on $\left(\mathbb{R}^{n}, \mathrm{~d}_{\mathrm{Eucl}}, \mu\right)$ that will play a fundamental role in the forthcoming discussion:

(a) The $G_{\mu}$-structure $\left(C_{c}^{\infty}\left(\mathbb{R}^{n}\right), \nabla\right)$.

(b) The $G_{\mathrm{AM}}$-structure $\left(\operatorname{LIP}_{c}\left(\mathbb{R}^{n}\right), \nabla_{\mathrm{AM}}\right)$. Observe that

$$
\nabla_{\mathrm{AM}} f=\operatorname{pr}_{V_{\mu}}(\nabla f), \quad \text { for every } f \in C_{c}^{\infty}\left(\mathbb{R}^{n}\right) .
$$

The axioms defining a $G$-structure are satisfied both in (a) and in (b), as a consequence of the results contained in Sects. 2.2 and 3.1, respectively.

Much like in the case of Sobolev spaces via test plans and minimal weak upper gradients, any $G$-structure naturally comes with a unique minimal object, called the minimal $G$-gradient:

Definition 3.11 (G-gradient) Let $\mu \geq 0$ be a Radon measure on $\mathbb{R}^{n}$ and $(\mathcal{V}, \bar{\nabla})$ a $G$ -structure on $\left(\mathbb{R}^{n}, \mathrm{~d}_{\mathrm{Eucl}}, \mu\right)$. Fix $f \in L^{2}(\mu)$. Then, we say that $f$ admits a $G$-gradient $\underline{v} \in L^{2}\left(\mathbb{R}^{n}, \mathbb{R}^{n} ; \mu\right)$ provided there exists a sequence $\left(f_{i}\right)_{i} \subseteq \mathcal{V}$ such that

$$
\begin{aligned}
& f_{i} \rightarrow f, \quad \text { strongly in } L^{2}(\mu), \\
& \bar{\nabla} f_{i} \rightarrow \underline{v}, \quad \text { strongly in } L^{2}\left(\mathbb{R}^{n}, \mathbb{R}^{n} ; \mu\right) \text {. }
\end{aligned}
$$


We denote by $G(f)$ the closed affine subspace of $L^{2}\left(\mathbb{R}^{n}, \mathbb{R}^{n} ; \mu\right)$ made of all $G$-gradients of $f$. The (unique) element of $G(f)$ of minimal norm is called the minimal $G$-gradient of $f$.

Observe that $\bar{\nabla} f \in G(f)$ for every $f \in \mathcal{V}$, as one can see by taking $f_{i}:=f$ for every $i \in \mathbb{N}$.

Remark 3.12 Note that the space $G(0)$ is closed under multiplication by $C_{c}^{\infty}\left(\mathbb{R}^{n}\right)$-functions: given any $g \in C_{c}^{\infty}\left(\mathbb{R}^{n}\right)$ and $\underline{v} \in G(0)$, it holds that $g \underline{v} \in G(0)$. Indeed, if $\left(f_{i}\right)_{i} \subseteq C_{c}^{\infty}\left(\mathbb{R}^{n}\right)$ is a sequence satisfying $f_{i} \rightarrow \overline{0}$ in $L^{2}(\mu)$ and $\bar{\nabla} f_{i} \rightarrow \underline{v}$ in $L^{2}\left(\mathbb{R}^{n}, \mathbb{R}^{n} ; \mu\right)$, then $g f_{i} \rightarrow 0$ in $L^{2}(\mu)$ and $\bar{\nabla}\left(g f_{i}\right)=g \bar{\nabla} f_{i}+f_{i} \bar{\nabla} g \rightarrow g v$ in $L^{2}\left(\mathbb{R}^{n}, \mathbb{R}^{n} ; \mu\right)$. In particular, we deduce from Lemma 2.24 that the space $G(0)$ is an $L^{2}(\mu)$-normed $L^{\infty}(\mu)$-submodule of $L^{2}\left(\mathbb{R}^{n}, \mathbb{R}^{n} ; \mu\right)$.

Definition 3.13 Let $\mu$ be a Radon measure on $\mathbb{R}^{n}$ and $(\mathcal{V}, \bar{\nabla})$ a $G$-structure on $\left(\mathbb{R}^{n}, \mathrm{~d}_{\text {Eucl }}, \mu\right)$. Then, we define $W_{G}$ as the unique element of $\mathscr{D}_{n}(\mu)$ such that

$$
\Gamma\left(W_{G}\right)=G(0) \text {. }
$$

Notice that the previous definition is meaningful as a consequence of Remark 3.12.

\subsubsection{Alternative characterisations of the tangent distribution}

The following two results show that $G$-structures can be used to provide an alternative notion of Sobolev space, which turns out to be fully equivalent to the approach via test plans.

Theorem 3.14 (Alternative characterisation of $W^{1,2}$ ) Let $\mu \geq 0$ be a Radon measure on $\mathbb{R}^{n}$ and let $(\mathcal{V}, \bar{\nabla})$ be a $G$-structure on $\left(\mathbb{R}^{n}, \mathrm{~d}_{\text {Eucl }}, \mu\right)$. Then

$$
W^{1,2}\left(\mathbb{R}^{n}, \mu\right)=\left\{f \in L^{2}(\mu) \mid G(f) \neq \emptyset\right\} .
$$

Moreover, for every $f \in W^{1,2}\left(\mathbb{R}^{n}, \mu\right)$ it holds that the minimal weak upper gradient $\left|D_{\mu} f\right|$ coincides (in the $\mu$-a.e. sense) with the pointwise norm of the minimal G-gradient of $f$.

Proof First of all, let us fix any function $f \in W^{1,2}\left(\mathbb{R}^{n}, \mu\right)$. We claim that $G(f) \neq \emptyset$ and that there exists an element $\underline{v} \in G(f)$ such that $\||\underline{v}|\|_{L^{2}(\mu)} \leq\left\|\left|D_{\mu} f\right|\right\|_{L^{2}(\mu)}$. In order to prove it, choose a sequence $\left(f_{i}\right)_{i} \subseteq \mathcal{V}$ such that $f_{i} \rightarrow f$ and $\left|\bar{\nabla} f_{i}\right| \rightarrow\left|D_{\mu} f\right|$ in $L^{2}(\mu)$, whose existence is observed in Remark 3.9. Up to a not relabelled subsequence, it holds that $\bar{\nabla} f_{i} \rightarrow \underline{v}$ weakly in $L^{2}\left(\mathbb{R}^{n}, \mathbb{R}^{n} ; \mu\right)$ for some vector field $\underline{v} \in L^{2}\left(\mathbb{R}^{n}, \mathbb{R}^{n} ; \mu\right)$. By Banach-Saks theorem we know that (up to taking a further subsequence) it holds that the functions $g_{i}:=\frac{1}{i} \sum_{j=1}^{i} f_{j} \in \mathcal{V}$ satisfy $g_{i} \rightarrow f$ in $L^{2}(\mu)$ and $\bar{\nabla} g_{i} \rightarrow \underline{v}$ strongly in $L^{2}\left(\mathbb{R}^{n}, \mathbb{R}^{n} ; \mu\right)$, which yields $\underline{v} \in G(f)$. It also holds that $\||\underline{v}|\|_{L^{2}(\mu)}=\lim _{i}\left\|\left|\bar{\nabla} g_{i}\right|\right\|_{L^{2}(\mu)} \leq \lim _{i} \frac{1}{i} \sum_{j=1}^{i}\left\|\left|\bar{\nabla} f_{j}\right|\right\|_{L^{2}(\mu)}=\left\|\mid D_{\mu} f \overline{\mid}\right\|_{L^{2}(\mu)}$.

Conversely, let us suppose that $f \in L^{2}(\mu)$ satisfies $G(f) \neq \emptyset$. Fix an element $\underline{v} \in G(f)$. Pick any sequence $\left(f_{i}\right)_{i} \subseteq \mathcal{V}$ such that $f_{i} \rightarrow f$ in $L^{2}(\mu)$ and $\bar{\nabla} f_{i} \rightarrow \underline{v}$ in $L^{2}\left(\mathbb{R}^{n}, \mathbb{R}^{n} ; \bar{\mu}\right)$. In particular, $\left|\bar{\nabla} f_{i}\right| \rightarrow|\underline{v}|$ in $L^{2}(\mu)$. Since $\left|\bar{\nabla} f_{i}\right|$ is a weak upper gradient of $f_{i}$ for every $i \in \mathbb{N}$, we deduce from Proposition 2.11 that $f \in W^{1,2}\left(\mathbb{R}^{n}, \mu\right)$ and $\left|D_{\mu} f\right| \leq|\underline{v}|$ holds $\mu$-a.e. in $\mathbb{R}^{n}$. All in all, the proof of the statement is finally achieved. 
Proposition 3.15 Let $\mu \geq 0$ be a Radon measure on $\mathbb{R}^{n}$ and let $(\mathcal{V}, \bar{\nabla})$ be a $G$-structure on $\left(\mathbb{R}^{n}, \mathrm{~d}_{\text {Eucl }}, \mu\right)$. Then, for any $f \in \mathcal{V}$ it holds that $\operatorname{pr}_{W_{G}^{\perp}}(\bar{\nabla} f)$ is the minimal G-gradient of $f$. In particular, $\left|\mathrm{pr}_{W_{G}^{\perp}}(\bar{\nabla} f)\right|$ is the minimal weak upper gradient of $f$.

Proof We claim that for any element $\underline{v} \in G(f)$ it holds that $\operatorname{pr}_{W_{G}^{\perp}}(\underline{v})$ belongs to $G(f)$ and is independent of $\underline{v}$. First, recall that $\bar{\nabla} f \in G(f)$. Since Lemma 2.24 yields $\operatorname{pr}_{W_{G}}(\bar{\nabla} f) \in G(0)$, there exists $\left(g_{i}\right)_{i} \subseteq \mathcal{V}$ such that $g_{i} \rightarrow 0$ in $L^{2}(\mu)$ and $\bar{\nabla} g_{i} \rightarrow \operatorname{pr}_{W_{G}}(\bar{\nabla} f)$ in $L^{2}\left(\mathbb{R}^{n}, \mathbb{R}^{n} ; \mu\right)$. Hence, the sequence $\left(f-g_{i}\right)_{i} \subseteq \mathcal{V}$ satisfies $f-g_{i} \rightarrow f$ in $L^{2}(\mu)$ and $\bar{\nabla}\left(f-g_{i}\right) \rightarrow \operatorname{pr}_{W_{G}^{\perp}}(\bar{\nabla} f)$ in $L^{2}\left(\mathbb{R}^{n}, \mathbb{R}^{n} ; \mu\right)$, yielding $\operatorname{pr}_{W_{G}^{\perp}}(\bar{\nabla} f) \in G(f)$. Furthermore, let $\underline{v} \in G(f)$ be fixed. Pick any sequence $\left(f_{i}\right)_{i} \subseteq \mathcal{V}$ such that $f_{i} \rightarrow f$ in $L^{2}(\mu)$ and $\bar{\nabla} f_{i} \rightarrow v$ in $L^{2}\left(\mathbb{R}^{n}, \mathbb{R}^{n} ; \mu\right)$. This implies that $\left(f-f_{i}\right)_{i} \subseteq \mathcal{V}$ satisfies $f-f_{i} \rightarrow 0$ in $L^{2}(\mu)$ and $\bar{\nabla}\left(f-f_{i}\right) \rightarrow \bar{\nabla} f-\underline{v}$ in $L^{2}\left(\mathbb{R}^{n}, \mathbb{R}^{n} ; \mu\right)$. Consequently, we conclude that $\bar{\nabla} f-\underline{v} \in G(0)$, thus $\bar{\nabla} f(x)-\underline{v}(x) \in W_{G}^{-}(x)$ for $\mu$-a.e. $x \in \mathbb{R}^{n}$. This means that $\operatorname{pr}_{W_{G}^{\perp}}(\bar{\nabla} f)-\operatorname{pr}_{W_{G}^{\perp}}(\underline{v})=\operatorname{pr}_{W_{G}^{\perp}}(\bar{\nabla} f-\underline{v})=0$. All in all, the claim is proven.

Now the first part of the statement readily follows: given any $\underline{v} \in G(f)$, it holds that

$$
\left\|\operatorname{pr}_{W_{G}^{\perp}}(\bar{\nabla} f)\right\|_{L^{2}\left(\mathbb{R}^{n}, \mathbb{R}^{n} ; \mu\right)}=\left\|\operatorname{pr}_{W_{G}^{\perp}}(\underline{v})\right\|_{L^{2}\left(\mathbb{R}^{n}, \mathbb{R}^{n} ; \mu\right)} \leq\|\underline{v}\|_{L^{2}\left(\mathbb{R}^{n}, \mathbb{R}^{n} ; \mu\right)} .
$$

Therefore, we finally conclude that $\operatorname{pr}_{W_{G}^{\perp}}(\bar{\nabla} f)$ is the minimal $G$-gradient of $f$. The last part of the statement now follows from Theorem 3.14, thus the proof is complete.

We are now ready to state and prove the main result of this section. It says that the tangent distribution $T_{\mu}$ can be expressed either in terms of the domain of the distributional divergence $\underline{\operatorname{div}}_{\mu}$, or of the $G_{\mu}$-structure. We point out that, to the best of our knowledge, the equivalence between these two approaches (namely items ii) and iii) of the following result) was previously not known; one of the two implications is proved in [33, end of Section 1].

Theorem 3.16 (Alternative characterisations of $T_{\mu}$ ) Let $\mu \geq 0$ be a Radon measure on $\mathbb{R}^{n}$. Then, the tangent distribution $T_{\mu}$ can be equivalently characterised in the following ways:

(i) $T_{\mu}$ is the unique minimal element of $\mathscr{D}_{n}(\mu)$ with the property that for any test plan $\pi$ on $\left(\mathbb{R}^{n}, \mathrm{~d}_{\text {Eucl }}, \mu\right)$ it holds $\dot{\gamma}_{t} \in T_{\mu}\left(\gamma_{t}\right)$ for $\left(\pi \otimes \mathcal{L}_{1}\right)$-a.e. $(\gamma, t) \in A C^{2}\left([0,1], \mathbb{R}^{n}\right) \times[0,1]$.

(ii) $T_{\mu}$ is the unique minimal element of $\mathscr{D}_{n}(\mu)$ with the property that for any $\underline{v} \in D\left(\underline{\operatorname{div}}_{\mu}\right)$ it holds that $\underline{v}(x) \in T_{\mu}(x)$ for $\mu$-a.e. $x \in \mathbb{R}^{n}$. Equivalently, $\iota_{\mu}\left(L_{\mu}^{2}\left(T \mathbb{R}^{n}\right)\right)=\operatorname{cl} D\left(\underline{\operatorname{div}}_{\mu}\right)$.

(iii) It holds that $\bar{T}_{\mu}=W_{\mu}^{\perp}$, where $W_{\mu}:=W_{G_{\mu}}$ stands for the distribution on $\mathbb{R}^{n}$ associated with the $G_{\mu}$-structure $\left(C_{c}^{\infty}\left(\mathbb{R}^{n}\right), \nabla\right)$, which is described in item a) of Example 3.10.

In items i) and ii), minimality has to be intended with respect to the partial order $\leq$ on $\mathscr{D}_{n}(\mu)$.

Proof We subdivide the proof into several steps:

STEP 1. First of all, we claim that $T_{\mu} \leq W_{\mu}^{\perp}$. This would follow from the inclusions

$$
\iota_{\mu}\left(L_{\mu}^{2}\left(T \mathbb{R}^{n}\right)\right) \subseteq \operatorname{cl} D\left(\underline{\operatorname{div}}_{\mu}\right) \subseteq G_{\mu}(0)^{\perp} .
$$

Indeed, by using (3.13), (3.5), (3.12), and Remark 2.26, we deduce that $\Gamma\left(T_{\mu}\right) \subseteq \Gamma\left(W_{\mu}^{\perp}\right)$, whence $T_{\mu} \leq W_{\mu}^{\perp}$ by the last part of the statement of Proposition 2.22. To prove the first inclusion in (3.13), recall that $\operatorname{cl} D\left(\operatorname{div}_{\mu}\right)=L_{\mu}^{2}\left(T \mathbb{R}^{n}\right)$ by Lemma 2.13 and notice that 


$$
l_{\mu}\left(L_{\mu}^{2}\left(T \mathbb{R}^{n}\right)\right)=\iota_{\mu}\left(\operatorname{cl} D\left(\operatorname{div}_{\mu}\right)\right) \subseteq \operatorname{cl} \iota_{\mu}\left(D\left(\operatorname{div}_{\mu}\right)\right) \stackrel{(1.25)}{\subseteq} \operatorname{cl} D\left(\underline{\operatorname{div}}_{\mu}\right)
$$

To prove the second inclusion in (3.13), it clearly suffices to show that $D\left(\underline{\operatorname{div}}_{\mu}\right) \subseteq G_{\mu}(0)^{\perp}$. To this aim, fix $\underline{v} \in D\left(\underline{\operatorname{div}}_{\mu}\right)$ and $\underline{w} \in G_{\mu}(0)$. Choose any $\left(f_{i}\right)_{i} \subseteq C_{c}^{\infty}\left(\mathbb{R}^{n}\right)$ such that $f_{i} \rightarrow 0$ in $L^{2}(\mu)$ and $\nabla f_{i} \rightarrow \underline{w}$ in $L^{2}\left(\mathbb{R}^{n}, \mathbb{R}^{n} ; \bar{\mu}\right)$. Therefore, we have that

$$
\int \underline{v} \cdot \underline{w} \mathrm{~d} \mu=\lim _{i \rightarrow \infty} \int \underline{v} \cdot \nabla f_{i} \mathrm{~d} \mu=-\lim _{i \rightarrow \infty} \int f_{i} \underline{\operatorname{div}}_{\mu}(\underline{v}) \mathrm{d} \mu=0 .
$$

By arbitrariness of $\underline{v}$ and $\underline{w}$, we conclude that $D\left(\underline{\operatorname{div}}_{\mu}\right) \subseteq G_{\mu}(0)^{\perp}$, so that (3.13) is proven.

STEP 2. Let $V \bar{\in} \mathscr{D}_{n}(\bar{\mu})$ be a distribution on $\mathbb{R}^{n}$ such that for any test plan $\pi$ on $\left(\mathbb{R}^{n}, \mathrm{~d}_{\text {Eucl }}, \mu\right)$ it holds that $\dot{\gamma}_{t} \in V\left(\gamma_{t}\right)$ for $\left(\boldsymbol{\pi} \otimes \mathcal{L}_{1}\right)$-a.e. $(\gamma, t)$. Then, we claim that $W_{\mu}^{\perp} \leq V$.

First, from STEP 1 and Lemma 3.6 we know that $W_{\mu}^{\perp} \cap V \in \mathscr{D}_{n}(\mu)$ satisfies the same property as $V$, i.e. for any test plan $\pi$ one has $\dot{\gamma}_{t} \in W_{\mu}\left(\gamma_{t}\right)^{\perp} \cap V\left(\gamma_{t}\right)$ for $\left(\boldsymbol{\pi} \otimes \mathcal{L}_{1}\right)$-a.e. $(\gamma, t)$. Let $W \in \mathscr{D}_{n}(\mu)$ be defined so that $W(x)$ is the orthogonal complement of $W_{\mu}(x)^{\perp} \cap V(x)$ in $W_{\mu}(x)^{\perp}$ for $\mu$-a.e. point $x \in \mathbb{R}^{n}$. Given any function $f \in C_{c}^{\infty}\left(\mathbb{R}^{n}\right)$, it holds that $\left|\operatorname{pr}_{W_{\mu}^{\perp} \cap V}(\nabla f)\right|$ is a weak upper gradient of $f$ by Lemma 3.7, while $\left|\operatorname{pr}_{W_{\mu}^{\perp}}(\nabla f)\right|$ is the minimal weak upper gradient of $f$ by Proposition 3.15. This implies that $\left|\operatorname{pr}_{W_{\mu}^{\perp} \cap V}(\nabla f)\right|=\left|\operatorname{pr}_{W_{\mu}^{\perp}}(\nabla f)\right|$ holds $\mu$-a.e. in $\mathbb{R}^{n}$ for every $f \in C_{c}^{\infty}\left(\mathbb{R}^{n}\right)$, thus accordingly we might conclude that

$$
\left|\operatorname{pr}_{W}(\nabla f)\right|^{2}=\left|\operatorname{pr}_{W_{\mu}^{\perp}}(\nabla f)\right|^{2}-\left|\operatorname{pr}_{W_{\mu}^{\perp} \cap V}(\nabla f)\right|^{2}=0 \quad \mu \text {-a.e., } \quad \text { for every } f \in C_{c}^{\infty}\left(\mathbb{R}^{n}\right) .
$$

Given that $\left\{\nabla f: f \in C_{c}^{\infty}\left(\mathbb{R}^{n}\right)\right\}$ generates $L^{2}\left(\mathbb{R}^{n}, \mathbb{R}^{n} ; \mu\right)$ on $\mathbb{R}^{n}$, we deduce that the image of $\operatorname{pr}_{W}$ coincides with $\{0\}$, thus necessarily $W=\{0\}$. This means that $W_{\mu}(x)^{\perp} \cap V(x)=W_{\mu}(x)^{\perp}$ for $\mu$-a.e. point $x \in \mathbb{R}^{n}$, which grants that $W_{\mu}^{\perp} \leq V$. Hence, the claim is proven.

STEP 3. By Lemma 3.6 we know that $T_{\mu}$ satisfies the property in item (i), whence by STEPS 1 and 2 we see that $T_{\mu}=W_{\mu}^{\perp}$ is the (unique) minimal distribution on $\mathbb{R}^{n}$ having this property, proving items iii) and i). Moreover, notice that $\iota_{\mu}\left(L_{\mu}^{2}\left(T \mathbb{R}^{n}\right)\right)=\operatorname{cl} D\left(\underline{\operatorname{div}}_{\mu}\right)=G_{\mu}(0)^{\perp}$ follows from (3.13) and the identity $T_{\mu}=W_{\mu}^{\perp}$, thus item (ii) is proven as well.

Note that by combining Lemma 2.20 with item (ii) of the previous theorem, we obtain that

$$
l_{\mu}\left(D\left(\operatorname{div}_{\mu}\right)\right)=D\left(\underline{\operatorname{div}}_{\mu}\right), \quad \text { for every Radon measure } \mu \geq 0 \text { on } \mathbb{R}^{n} .
$$

As another immediate consequence of Theorem 3.16, we also see that the tangent distribution is always contained in the Alberti-Marchese distribution:

Corollary 3.17 Let $\mu \geq 0$ be a Radon measure on $\mathbb{R}^{n}$. Then, it holds that

$$
T_{\mu} \leq V_{\mu}
$$

Proof Combine item (i) of Theorem 3.3 with item (i) of Theorem 3.16.

Remark 3.18 It might happen that $T_{\mu} \neq V_{\mu}$. For instance, let $C$ be a fat Cantor set in $\mathbb{R}$ and consider $\mu:=\left.\mathcal{L}^{1}\right|_{C}$. Then, $V_{\mu}(x)=\mathbb{R}$ for $\mu$-a.e. $x \in \mathbb{R}$ by Remark 3.2, while $T_{\mu}(x)=\{0\}$ for $\mu$-a.e. $x \in \mathbb{R}$ as a consequence of the fact that the support of $\mu$ is totally disconnected, thus $W^{1,2}(\mathbb{R}, \mu)=L^{2}(\mu)$ and $\left|D_{\mu} f\right|=0$ holds $\mu$-a.e. for every $f \in W^{1,2}(\mathbb{R}, \mu)$. 


\subsection{Identification of the minimal weak upper gradient}

Once we have the equivalent characterisations of the Sobolev space and of the tangent distribution at our disposal, we can identify the minimal weak upper gradient of every given Lipschitz function. First, we deal with smooth functions (in Proposition 3.19), then we pass to general Lipschitz functions (in Theorem 3.20). A consequence of Proposition 3.19—namely the fact that $\left|D_{\mu} f\right|=\left|\mathrm{pr}_{T_{\mu}}(\nabla f)\right|$ holds for every $f \in C_{c}^{\infty}\left(\mathbb{R}^{n}\right)$ —was already proven by S. Di Marino in [16, Theorem 7.4.8].

Proposition 3.19 Let $\mu \geq 0$ be a Radon measure on $\mathbb{R}^{n}$. Then, for every $f \in W^{1,2}\left(\mathbb{R}^{n}, \mu\right)$ it holds that $l_{\mu}\left(\nabla_{\mu} f\right)$ is the minimal $G_{\mu^{-}}$gradient of $f$. In particular, we have that

$$
\iota_{\mu}\left(\nabla_{\mu} f\right)=\operatorname{pr}_{T_{\mu}}(\nabla f), \quad \text { for every } f \in C_{c}^{\infty}\left(\mathbb{R}^{n}\right) .
$$

Proof First of all, let us prove (3.15). Fix any $f \in C_{c}^{\infty}\left(\mathbb{R}^{n}\right)$. Choose any $v \in L_{\mu}^{2}\left(T \mathbb{R}^{n}\right)$ such that $l_{\mu}(v)=\operatorname{pr}_{T_{\mu}}(\nabla f)$. Therefore, for every $g \in C_{c}^{\infty}\left(\mathbb{R}^{n}\right)$ it holds that

$$
\begin{aligned}
\mathrm{d}_{\mu} g\left(\nabla_{\mu} f\right) & =\mathrm{d}_{\mu} f\left(\nabla_{\mu} g\right) \stackrel{(1.21)}{=} \mathrm{d} f\left(\iota_{\mu}\left(\nabla_{\mu} g\right)\right)=\nabla f \cdot \iota_{\mu}\left(\nabla_{\mu} g\right)=\operatorname{pr}_{T_{\mu}}(\nabla f) \cdot \iota_{\mu}\left(\nabla_{\mu} g\right) \\
& =\iota_{\mu}(v) \cdot \iota_{\mu}\left(\nabla_{\mu} g\right)=\left\langle v, \nabla_{\mu} g\right\rangle=\mathrm{d}_{\mu} g(v) \stackrel{(1.21)}{=} \mathrm{d} g\left(\iota_{\mu}(v)\right)=\mathrm{d} g\left(\mathrm{pr}_{T_{\mu}}(\nabla f)\right) .
\end{aligned}
$$

In light of Remark 2.17, we can conclude that $l_{\mu}\left(\nabla_{\mu} f\right)=\operatorname{pr}_{T_{\mu}}(\nabla f)$, thus proving (3.15).

Let us now fix $f \in W^{1,2}\left(\mathbb{R}^{n}, \mu\right)$. By Corollary 2.19 there is a sequence $\left(f_{i}\right)_{i} \subseteq C_{c}^{\infty}\left(\mathbb{R}^{n}\right)$ such that $f_{i} \rightarrow f,\left|D_{\mu} f_{i}\right| \rightarrow\left|D_{\mu} f\right|$, and $\left|\nabla f_{i}\right| \rightarrow\left|D_{\mu} f\right|$ in $L^{2}(\mu)$. Up to a not relabelled subsequence, we can also assume that $\nabla_{\mu} f_{i} \rightarrow v$ weakly in $L_{\mu}^{2}\left(T \mathbb{R}^{n}\right)$, for some $v \in L_{\mu}^{2}\left(T \mathbb{R}^{n}\right)$. By Banach-Saks theorem we can find a sequence $\left(g_{i}\right)_{i} \subseteq C_{c}^{\infty}\left(\mathbb{R}^{n}\right)$ such that $g_{i} \rightarrow f$ in $L^{2}(\mu), \nabla_{\mu} g_{i} \rightarrow v$ in $L_{\mu}^{2}\left(T \mathbb{R}^{n}\right)$, and $\varlimsup_{i}\left\|\left|\nabla g_{i}\right|\right\|_{L^{2}(\mu)} \leq\left\|\left|D_{\mu} f\right|\right\|_{L^{2}(\mu)}$. It follows from Proposition 2.11 that $v=\nabla_{\mu} f$, thus in particular $\left|D_{\mu} g_{i}\right| \rightarrow\left|D_{\mu} f\right|$ in $L^{2}(\mu)$. Item iii) of Theorem 3.16 yields

$$
\begin{aligned}
& \varlimsup_{i \rightarrow \infty} \int\left|\operatorname{pr}_{W_{\mu}}\left(\nabla g_{i}\right)\right|^{2} \mathrm{~d} \mu=\varlimsup_{i \rightarrow \infty}\left(\int\left|\nabla g_{i}\right|^{2} \mathrm{~d} \mu-\int\left|\operatorname{pr}_{T_{\mu}}\left(\nabla g_{i}\right)\right|^{2} \mathrm{~d} \mu\right) \\
& \stackrel{(2.15)}{=} \varlimsup_{i \rightarrow \infty} \int\left|\nabla g_{i}\right|^{2} \mathrm{~d} \mu-\lim _{i \rightarrow \infty} \int\left|D_{\mu} g_{i}\right|^{2} \mathrm{~d} \mu \\
& \leq \int\left|D_{\mu} f\right|^{2} \mathrm{~d} \mu-\int\left|D_{\mu} f\right|^{2} \mathrm{~d} \mu=0,
\end{aligned}
$$

whence $\operatorname{pr}_{W_{\mu}}\left(\nabla g_{i}\right) \rightarrow 0$ in $L^{2}\left(\mathbb{R}^{n}, \mathbb{R}^{n} ; \mu\right)$. Since $\nabla_{\mu} g_{i} \rightarrow \nabla_{\mu} f$ in $L_{\mu}^{2}\left(T \mathbb{R}^{n}\right)$ and $\iota_{\mu}$ is continuous, we can finally conclude that

$$
\nabla g_{i}=\operatorname{pr}_{T_{\mu}}\left(\nabla g_{i}\right)+\operatorname{pr}_{W_{\mu}}\left(\nabla g_{i}\right) \stackrel{(2.15)}{=} l_{\mu}\left(\nabla_{\mu} g_{i}\right)+\operatorname{pr}_{W_{\mu}}\left(\nabla g_{i}\right) \rightarrow l_{\mu}\left(\nabla_{\mu} f\right), \quad \text { in } L^{2}\left(\mathbb{R}^{n}, \mathbb{R}^{n} ; \mu\right) .
$$

This means that $l_{\mu}\left(\nabla_{\mu} f\right) \in G_{\mu}(f)$. Given that $\left|l_{\mu}\left(\nabla_{\mu} f\right)\right|=\left|D_{\mu} f\right|$ holds $\mu$-a.e. in $\mathbb{R}^{n}$, we infer from Theorem 3.14 that $l_{\mu}\left(\nabla_{\mu} f\right)$ is the minimal $G_{\mu}$-gradient of $f$. The proof is complete.

Theorem 3.20 (Minimal weak upper gradient of Lipschitz functions) Let $\mu \geq 0$ be $a$ Radon measure on $\mathbb{R}^{n}$. Then, it holds that 


$$
\left|D_{\mu} f\right|=\left|\operatorname{pr}_{T_{\mu}}\left(\nabla_{\mathrm{AM}} f\right)\right| \quad \mu \text {-a.e., } \quad \text { for every } f \in \operatorname{LIP}_{c}\left(\mathbb{R}^{n}\right) .
$$

Proof Consider the $G_{\mu}$-structure and the $G_{\mathrm{Am}}$-structure, which were defined in items (a) and (b) of Example 3.10, respectively. For brevity, we call $W_{\mathrm{AM}}:=W_{G_{\mathrm{AM}}}$. First, we prove that

$$
W_{\mathrm{AM}}^{\perp} \cap V_{\mu}=T_{\mu} .
$$

In order to show one inclusion, fix $\underline{v} \in G_{\mathrm{AM}}(0)^{\perp} \cap \Gamma\left(V_{\mu}\right)$ and $\underline{w} \in G_{\mu}(0)$. Let us pick any sequence $\left(f_{i}\right)_{i} \subseteq C_{c}^{\infty}\left(\mathbb{R}^{n}\right)$ satisfying $f_{i} \rightarrow 0$ in $L^{2}(\mu)$ and $\nabla \overline{f_{i}} \rightarrow \underline{w}$ in $L^{2}\left(\mathbb{R}^{n}, \mathbb{R}^{n} ; \mu\right)$. The latter convergence, together with (3.11), yields $\left.\nabla_{\mathrm{AM}} f_{i}=\operatorname{pr}_{V_{\mu}} \overline{(\nabla} f_{i}\right) \rightarrow \operatorname{pr}_{V_{\mu}}(\underline{w})$ in $L^{2}\left(\mathbb{R}^{n}, \mathbb{R}^{n} ; \mu\right)$, which gives $\operatorname{pr}_{V_{\mu}}(\underline{w}) \in G_{\mathrm{AM}}(0)$. Since $\underline{v} \in G_{\mathrm{AM}}(0)^{\perp} \cap \Gamma\left(V_{\mu}\right)$, we get that $\underline{v} \cdot \underline{w}=\underline{v} \cdot \operatorname{pr}_{V_{\mu}}(\underline{w})=0$ holds $\mu$-a.e., which implies $G_{\mathrm{AM}}^{-}(0)^{\perp} \cap \Gamma\left(V_{\mu}\right) \subseteq G_{\mu}(0)^{\perp}$ and thus $\bar{W}_{\mathrm{AM}}^{\perp} \cap \bar{V}_{\mu} \leq W_{\mu}^{\perp}=T_{\mu}$.

To prove the converse inclusion, let us consider the orthogonal complement $Z$ of $W_{\mathrm{AM}}^{\perp} \cap V_{\mu}$ in $T_{\mu}$, namely $Z:=\left(W_{\mathrm{AM}}^{\perp} \cap V_{\mu}\right)^{\perp} \cap T_{\mu}$. Notice that for any $f \in C_{c}^{\infty}\left(\mathbb{R}^{n}\right)$ we have that

$$
\left|\operatorname{pr}_{T_{\mu}}\left(\nabla_{\mathrm{AM}} f\right)\right|^{2}=\left|\operatorname{pr}_{W_{\mathrm{AM}}^{\perp} \cap V_{\mu}}\left(\nabla_{\mathrm{AM}} f\right)\right|^{2}+\left|\operatorname{pr}_{Z}\left(\nabla_{\mathrm{AM}} f\right)\right|^{2}, \quad \text { in the } \mu \text {-a.e. sense. }
$$

By applying Proposition 3.15 to the $G_{\mu}$-structure and the $G_{\mathrm{AM}}$-structure, we obtain that

$$
\begin{aligned}
\left|\operatorname{pr}_{T_{\mu}}\left(\nabla_{\mathrm{AM}} f\right)\right| \stackrel{(2.14)}{=}\left|\operatorname{pr}_{T_{\mu}}(\nabla f)\right|=\left|\operatorname{pr}_{W_{\mu}^{\perp}}(\nabla f)\right|=\left|D_{\mu} f\right|, \\
\left|\operatorname{pr}_{W_{\mathrm{AM}}^{\perp} \cap V_{\mu}}\left(\nabla_{\mathrm{AM}} f\right)\right|=\left|\operatorname{pr}_{W_{\mathrm{AM}}^{\perp}}\left(\nabla_{\mathrm{AM}} f\right)\right|=\left|D_{\mu} f\right|,
\end{aligned}
$$

respectively. By plugging (3.18) into (3.17), we deduce that $\left|\operatorname{pr}_{Z}(\nabla f)\right|=\left|\operatorname{pr}_{Z}\left(\nabla_{\mathrm{AM}} f\right)\right|=0$ holds $\mu$-a.e. for all $f \in C_{c}^{\infty}\left(\mathbb{R}^{n}\right)$. Since $\left\{\nabla f: f \in C_{c}^{\infty}\left(\mathbb{R}^{n}\right)\right\}$ generates $L^{2}\left(\mathbb{R}^{n}, \mathbb{R}^{n} ; \mu\right)$ on $\mathbb{R}^{n}$, we conclude that $Z=\{0\}$, which means that the identity in (3.16) is verified.

Now fix any function $f \in \operatorname{LIP}_{c}\left(\mathbb{R}^{n}\right)$. We know that $\left|\mathrm{pr}_{W_{\mathrm{AM}}^{\perp}}\left(\nabla_{\mathrm{AM}} f\right)\right|$ is the minimal weak upper gradient of $f$ by Proposition 3.15. Since it also holds that

$$
\left|\mathrm{pr}_{T_{\mu}}\left(\nabla_{\mathrm{AM}} f\right)\right| \stackrel{(2.16)}{=}\left|\operatorname{pr}_{W_{\mathrm{AM}}^{\perp} \cap V_{\mu}}\left(\nabla_{\mathrm{AM}} f\right)\right|=\left|\mathrm{pr}_{W_{\mathrm{AM}}^{\perp}}\left(\nabla_{\mathrm{AM}} f\right)\right|, \quad \text { in the } \mu \text {-a.e. sense, }
$$

we finally conclude that $\left|\operatorname{pr}_{T_{\mu}}\left(\nabla_{\mathrm{AM}} f\right)\right|$ is the minimal weak upper gradient of $f$.

It readily follows from Theorem 3.20 that those measures $\mu$ on $\mathbb{R}^{n}$ for which minimal weak upper gradient and local Lipschitz constant always coincide can be explicitly characterised in terms of the tangent distribution $T_{\mu}$, as the next result shows.

Corollary 3.21 Let $\mu \geq 0$ be a Radon measure on $\mathbb{R}^{n}$. Then, the following are equivalent:

i) $\left|D_{\mu} f\right|=\operatorname{lip}(f)$ holds $\mu$-a.e., for every $f \in \operatorname{LIP}_{c}\left(\mathbb{R}^{n}\right)$,

ii) $T_{\mu}(x)=\mathbb{R}^{n}$, for $\mu$-a.e. $x \in \mathbb{R}^{n}$.

Proof Suppose (i) holds. To prove (ii), we argue by contradiction: suppose there exists a Borel set $E \subseteq \mathbb{R}^{n}$ such that $\mu(E)>0$ and $T_{\mu}(x) \neq \mathbb{R}^{n}$ for $\mu$-a.e. $x \in E$. This means that for $\mu$ -a.e. point $x \in E$ there exists a vector $v \in \mathbb{Q}^{n}$ such that $v \notin T_{\mu}(x)$, in other words 


$$
E \subseteq \bigcup_{v \in \mathbb{Q}^{n}}\left\{x \in \mathbb{R}^{n} \mid v \notin T_{\mu}(x)\right\}, \quad \text { up to } \mu \text {-null sets. }
$$

Hence, there exist a vector $v \in \mathbb{Q}^{n}$ and a Borel set $F \subseteq E$ such that $\mu(F)>0$ and $\nu \notin T_{\mu}(x)$ for $\mu$-a.e. $x \in F$. Choose a radius $r>0$ such that $\mu\left(F \cap B_{r}(0)\right)>0$ and a function $f \in C_{c}^{\infty}\left(\mathbb{R}^{n}\right)$ satisfying $\nabla f(x)=v$ for every $x \in B_{r}(0)$. By using Proposition 3.19, we thus deduce that

$$
\left|D_{\mu} f\right|(x)=\left|\operatorname{pr}_{T_{\mu}}(\nabla f)\right|(x)=\left|\pi_{T_{\mu}(x)}(v)\right|<|v|=\operatorname{lip}(f)(x), \quad \text { for } \mu \text {-a.e. } x \in F \cap B_{r}(0) .
$$

This leads to a contradiction with (i), whence accordingly (ii) is proven.

Conversely, suppose (ii) holds. A fortiori, we have that $V_{\mu}(x)=\mathbb{R}^{n}$ for $\mu$-a.e. $x \in \mathbb{R}^{n}$ (recall Corollary 3.17), so that any given function $f \in \operatorname{LIP}_{c}\left(\mathbb{R}^{n}\right)$ is $\mu$-a.e. differentiable and thus $\left|\nabla_{\mathrm{AM}} f\right|=\operatorname{lip}(f)$ in the $\mu$-a.e. sense. Finally, by using Theorem 3.20 we obtain that

$$
\left|D_{\mu} f\right|=\left|\mathrm{pr}_{T_{\mu}}\left(\nabla_{\mathrm{AM}} f\right)\right|=\left|\nabla_{\mathrm{AM}} f\right|=\operatorname{lip}(f), \quad \text { holds } \mu \text {-a.e. on } \mathbb{R}^{n},
$$

proving the validity of (i).

\section{Some applications}

\subsection{Tangent fibres on the singular part}

In the structure theory of Radon measures on Euclidean spaces, a breakthrough is represented by the celebrated paper [15] by G. De Philippis and F. Rindler. A consequence of their main result is reported in Theorem 4.1.

In this section, we will combine the results by De Philippis-Rindler with our knowledge of the tangent distribution, in order to prove that for any Radon measure $\mu=\rho \mathcal{L}^{n}+\mu^{s}$ on $\mathbb{R}^{n}$ (where $\mu^{s} \perp \mathcal{L}^{n}$ ) it holds that $T_{\mu}(x) \neq \mathbb{R}^{n}$ for $\mu^{s}$-a.e. point $x \in \mathbb{R}^{n}$; see Theorem 4.6. This gives a positive answer to a variant of a question raised by Fragalà and Mantegazza [22, Remark 4.4]; the original problem was posed in terms of a different notion of tangent fibre. However, by adapting our arguments one can solve also their original open problem. We point out that neither the kind of results we will prove in this section, nor the techniques we will use, are really new. See, e.g. [14, 27, 31] for similar statements and arguments.

\subsubsection{Reminder on Euclidean 1-currents}

Recall that a 1-current $\mathrm{T}$ on $\mathbb{R}^{n}$ is a linear and continuous real-valued functional defined on the space of smooth, compactly supported 1-forms on $\mathbb{R}^{n}$. Its total mass $\mathbf{M}(\mathrm{T})$ is given by the supremum of $\mathrm{T}(\underline{\omega})$ among all smooth, compactly supported 1forms $\underline{\omega}$ on $\mathbb{R}^{n}$ that satisfy $|\underline{\omega}| \leq 1$ on all $\mathbb{R}^{n}$. If $\mathbf{M}(\mathrm{T})$ is finite, then $\mathrm{T}$ is an $\mathbb{R}^{n}$-valued Radon measure on $\mathbb{R}^{n}$, whence by using the Radon-Nikodým theorem one can find a finite, non-negative Borel measure $\|\mathrm{T}\|$ on $\mathbb{R}^{n}$ and a vector field $\overrightarrow{\mathrm{T}} \in L^{1}\left(\mathbb{R}^{n}, \mathbb{R}^{n} ;\|\mathrm{T}\|\right)$, with $|\overrightarrow{\mathrm{T}}(x)|=1$ for $\|\mathrm{T}\|$-a.e. point $x \in \mathbb{R}^{n}$, such that $\mathrm{T}=\overrightarrow{\mathrm{T}}\|\mathrm{T}\|$. The boundary $\partial \mathrm{T}$ of $\mathrm{T}$ is the 0 -current (i.e. the generalised function) on $\mathbb{R}^{n}$ which is defined as $\partial \mathrm{T}(f):=\mathrm{T}(\mathrm{d} f)$ for all $f \in C_{c}^{\infty}\left(\mathbb{R}^{n}\right)$. A 1 -current $\mathrm{T}$ on $\mathbb{R}^{n}$ is said to be normal provided $\mathbf{M}(\mathrm{T}), \mathbf{M}(\partial \mathrm{T})<+\infty$, 
where $\mathbf{M}(\partial \mathrm{T}):=\sup \left\{\partial \mathrm{T}(f): f \in C_{c}^{\infty}\left(\mathbb{R}^{n}\right),|f| \leq 1\right.$ on $\left.\mathbb{R}^{n}\right\}$. When the total mass $\mathbf{M}(\partial \mathrm{T})$ is finite, the 0 -current $\partial \mathrm{T}$ can be canonically identified with a (finite) signed measure on $\mathbb{R}^{n}$.

The following deep result, concerning the structure of normal 1-currents in the Euclidean space, has been proven by G. De Philippis and F. Rindler in the paper [15].

Theorem 4.1 Let $\mu \geq 0$ be a Radon measure on $\mathbb{R}^{n}$ and let $\mathrm{T}_{1}, \ldots, \mathrm{T}_{n}$ be normal 1-currents in $\mathbb{R}^{n}$ such that $\mu \ll\left\|\mathrm{T}_{i}\right\|$ for every $i=1, \ldots, n$. Suppose that

$$
\overrightarrow{\mathrm{T}}_{1}(x), \ldots, \overrightarrow{\mathrm{T}}_{n}(x) \in \mathbb{R}^{n} \text { are linearly independent, for } \mu \text {-a.e. } x \in \mathbb{R}^{n} .
$$

Then, it holds that $\mu \ll \mathcal{L}^{n}$.

As pointed out in [15], Theorem 4.1 has-among many others-the following consequence:

Theorem 4.2 (Weak converse of Rademacher theorem) Let $\mu$ be a Radon measure on $\mathbb{R}^{n}$ such that every function $f \in \operatorname{LIP}\left(\mathbb{R}^{n}\right)$ is $\mu$-a.e. differentiable. Then, it holds that $\mu \ll \mathcal{L}^{n}$.

In turn, the weak converse of Rademacher theorem readily implies that the Alberti-Marchese distribution has full rank if and only if the measure under consideration is absolutely continuous with respect to the Lebesgue measure:

Corollary 4.3 Let $\mu$ be a given Radon measure on $\mathbb{R}^{n}$. Then, it holds that

$$
V_{\mu}(x)=\mathbb{R}^{n}, \quad \text { for } \mu \text {-a.e. } x \in \mathbb{R}^{n} \Longleftrightarrow \mu \ll \mathcal{L}^{n} .
$$

Proof If $V_{\mu}(x)=\mathbb{R}^{n}$ for $\mu$-a.e. $x \in \mathbb{R}^{n}$, then every Lipschitz function is $\mu$-a.e. differentiable, whence $\mu \ll \mathcal{L}^{n}$ by Theorem 4.2. The converse implication is observed in Remark 3.2.

Example 4.4 (Vector fields with divergence as normal 1-currents) Let $\mu$ be a finite Borel measure on $\mathbb{R}^{n}$ and $\underline{v} \in D\left(\underline{\operatorname{div}}_{\mu}\right)$. Let us associate to $\underline{v}$ the 1 -current $\mathcal{I}(\underline{v})$ on $\mathbb{R}^{n}$, defined as

$$
\mathcal{I}(\underline{v})(\underline{\omega}):=\int \underline{\omega}(\underline{v}) \mathrm{d} \mu, \quad \text { for every smooth, compactly supported 1-form } \underline{\omega} \text { on } \mathbb{R}^{n} .
$$

Then, we claim that $\mathcal{I}(\underline{v})$ is a normal 1-current and that it satisfies

$$
\overline{\mathcal{I}(\underline{v})}=\mathbb{1}_{\{|\underline{v}|>0\}} \frac{\underline{v}}{|\underline{v}|}, \quad\|\mathcal{I}(\underline{v})\|=|\underline{v}| \mu, \quad \partial \mathcal{I}(\underline{v})=-\underline{\operatorname{div}}_{\mu}(\underline{v}) \mu .
$$

Indeed, the fact that $\mathbf{M}(\mathcal{I}(\underline{v}))<+\infty$, and the explicit formulae for $\overrightarrow{\mathcal{I}(\underline{v})}$ and $\|\mathcal{I}(\underline{v})\|$, are immediate consequences of (4.1), while it readily follows from the identity

$$
\partial \mathcal{I}(\underline{v})(f)=\mathcal{I}(\underline{v})(\mathrm{d} f)=\int \mathrm{d} f(\underline{v}) \mathrm{d} \mu=-\int f \underline{\operatorname{div}}_{\mu}(\underline{v}) \mathrm{d} \mu, \quad \text { for every } f \in C_{c}^{\infty}\left(\mathbb{R}^{n}\right),
$$

that the 0 -current $\partial \mathcal{I}(\underline{v})$ has finite total mass and satisfies $\partial \mathcal{I}(\underline{v})=-\underline{\operatorname{div}}_{\mu}(\underline{v}) \mu$. 


\subsubsection{The dimension drops on the singular part}

As a first step, we show that a given Radon measure on $\mathbb{R}^{n}$ must be absolutely continuous with respect to the Lebesgue measure $\mathcal{L}^{n}$ if restricted to any Borel set where the tangent module has maximal dimension.

Proposition 4.5 Let $\mu$ be a finite Borel measure on $\mathbb{R}^{n}$. Suppose $L_{\mu}^{2}\left(T \mathbb{R}^{n}\right)$ has dimension equal to $n$ on a Borel set $E \subseteq \mathbb{R}^{n}$. Then $\left.\mu\right|_{E} \ll \mathcal{L}^{n}$.

Proof Fix a countable dense subset $\mathcal{C}$ of $D\left(\underline{\operatorname{div}}_{\mu}\right)$. Given that $\Gamma\left(T_{\mu}\right)=\operatorname{cl} D\left(\underline{\operatorname{div}}_{\mu}\right)$ by item (ii) of Theorem 3.16 and div ${ }_{\mu}$ satisfies the Leibniz rule, we know from Lemma 2.24 that $T_{\mu}(x)$ coincides with $\operatorname{cl}\{\underline{v}(x): \underline{v} \in \mathcal{C}\}$ for $\mu$-a.e. $x \in \mathbb{R}^{n}$. In particular, Remark 3.5 grants that:

For $\mu$-a.e. $x \in E$, there exist $\underline{v}_{1}, \ldots, \underline{v}_{n} \in \mathcal{C}: \quad \operatorname{span}\left\{\underline{v}_{1}(x), \ldots, \underline{v}_{n}(x)\right\}=\mathbb{R}^{n}$.

Consider the family $\left(S_{k}\right)_{k \in \mathbb{N}}$ of all those subsets of $\mathcal{C}$ made exactly of $n$ elements. Given any $k \in \mathbb{N}$, we denote by $E_{k}$ the set of all points $x \in E$ such that $\underline{v}_{1}(x), \ldots, \underline{v}_{n}(x) \in \mathbb{R}^{n}$ are linearly independent, where $\left\{\underline{v}_{1}, \ldots, \underline{v}_{n}\right\}=S_{k}$. Then (4.2) grants that the Borel sets $E_{k}$ satisfy $\mu\left(E \backslash \bigcup_{k} E_{k}\right)=0$. Now fix any $k \in \mathbb{N} \in \mathbb{N}$ and call $S_{k}=\left\{\underline{v}_{1}, \ldots, \underline{v}_{n}\right\}$. Thanks to Example 4.4, the 1-currents $\mathcal{I}\left(\underline{v}_{1}\right), \ldots, \mathcal{I}\left(\underline{v}_{n}\right)$ are normal and satisfy $\left.\mu\right|_{E_{k}} \ll\left\|\mathcal{I}\left(\underline{v}_{i}\right)\right\|$ for all $i=1, \ldots, n$. Therefore, we conclude from Theorem 4.1 that $\left.\mu\right|_{E_{k}} \ll \mathcal{L}^{n}$ for all $k \in \mathbb{N}$, thus $\left.\mu\right|_{E} \ll \mathcal{L}^{n}$.

It is now easy to prove, as an immediate consequence of Proposition 4.5, that the tangent fibres cannot have dimension $n$ on the singular part of the measure $\mu$ under consideration.

Theorem 4.6 (Tangent fibres on the singular part) Let $\mu$ be a finite Borel measure on $\mathbb{R}^{n}$, with Lebesgue decomposition $\mu=\rho \mathcal{L}^{n}+\mu^{s}$. Then, it holds that

$$
\operatorname{dim} T_{\mu}(x)<n, \quad \text { for } \mu^{s} \text {-a.e. } x \in \mathbb{R}^{n} .
$$

Proof Fix a Borel set $B \subseteq \mathbb{R}^{n}$ such that $\mathcal{L}^{n}(B)=\mu^{s}\left(\mathbb{R}^{n} \backslash B\right)=0$. We argue by contradiction: suppose there is a Borel set $E \subseteq B$ such that $\mu^{s}(E)>0$ and $\operatorname{dim} T_{\mu}(x)=n$ for $\mu^{s}$ -a.e. $x \in E$. In particular, $\mu(E)>0$ and $\operatorname{dim} T_{\mu}(x)=n$ for $\mu$-a.e. $x \in E$. As observed in Remark 3.5, this means that the tangent module $L_{\mu}^{2}\left(T \mathbb{R}^{n}\right)$ has dimension $n$ on $E$. Therefore, Proposition 4.5 grants that $\left.\mu^{s}\right|_{E}=\left.\mu\right|_{E} \ll \mathcal{L}^{n}$. This leads to a contradiction, as $\mathcal{L}^{n}(E)=0$ but $\mu^{s}(E)>0$.

Remark 4.7 Actually, Theorem 4.6 holds for any non-negative Radon measure $\mu$ on $\mathbb{R}^{n}$. Indeed, given any $\bar{x} \in \operatorname{spt}(\mu)$ and $r>0$, it can be readily deduced from [23, Proposition 2.6] that $T_{\mu_{r}}(x)=T_{\mu}(x)$ is satisfied for $\mu$-a.e. $x \in B_{r}(\bar{x})$, where we set $\mu_{r}:=\left.\mu\right|_{B_{r}(\bar{x})}$. Moreover, notice that $\left(\mu_{r}\right)^{s}=\left.\mu^{s}\right|_{B_{r}(\bar{x})}$. Therefore, by applying Theorem 4.6 to the measures $\left(\mu_{k}\right)_{k \in \mathbb{N}}$ we deduce that $\mu$ itself satisfies (4.3), thus showing that in the statement of Theorem 4.6 the finiteness assumption on $\mu$ can be dropped.

Remark 4.8 (Weighted real line) As already mentioned in the introduction, the Sobolev space on weighted $\mathbb{R}$ has been fully understood by S. Di Marino and G. Speight in [20]. More specifically, they completely characterised the minimal weak upper gradient of any Lipschitz function $f \in W^{1,2}(\mathbb{R}, \mu)$, where $\mu$ is a given Radon measure on $\mathbb{R}$; see [20, 
Theorem 2]. We point out that our results imply a part (but not the whole) of their statement: Theorem 3.20 grants that $\left|D_{\mu} f\right|(x) \in\{0, \operatorname{lip}(f)(x)\}$ is satisfied for $\mu$-a.e. $x \in \mathbb{R}$, while Theorem 4.6 ensures that $T_{\mu}(x)=\{0\}$ and thus $\left|D_{\mu} f\right|(x)=0$ hold for $\mu^{s}$-a.e. $x \in \mathbb{R}$.

It is worth to isolate the following statement, which might be seen as a special case of Theorem 4.6 (or, alternatively, of Corollary 4.3).

Corollary 4.9 Let $\mu \geq 0$ be a Radon measure on $\mathbb{R}^{n}$ such that

$$
\left|D_{\mu} f\right|=\operatorname{lip}(f) \quad \mu \text {-a.e., } \quad \text { for every } f \in \operatorname{LIP}_{c}\left(\mathbb{R}^{n}\right) .
$$

Then, it holds that $\mu \ll \mathcal{L}^{n}$.

Proof By Corollary 3.21, we know that (4.4) is equivalent to $T_{\mu}(x)=\mathbb{R}^{n}$ for $\mu$-a.e. $x \in \mathbb{R}^{n}$. Therefore, it follows from Theorem 4.6 that $\mu^{s}=0$, which exactly means that $\mu \ll \mathcal{L}^{n}$.

Alternatively, one can argue as follows: since $T_{\mu}(x)=\mathbb{R}^{n}$ for $\mu$-a.e. $x \in \mathbb{R}^{n}$, we know a fortiori that $V_{\mu}(x)=\mathbb{R}^{n}$ for $\mu$-a.e. $x \in \mathbb{R}^{n}$, thus accordingly $\mu \ll \mathcal{L}^{n}$ by Corollary 4.3.

Remark 4.10 Suppose that $\mu$ is a Radon measure on $\mathbb{R}^{n}$ such that the resulting metric measure space $\left(\mathbb{R}^{n}, d_{\text {Eucl }}, \mu\right)$ is doubling and supports a weak $(1,2)$-Poincaré inequality, in the sense of [30]. Then, the property in (4.4) is satisfied, as proven by J. Cheeger in [13]. Therefore, it follows from Corollary 4.9 that the measure $\mu$ must be absolutely continuous with respect to $\mathcal{L}^{n}$. This fact was already proven by A. Schioppa in [37]. See also [14].

\subsection{A geometric characterisation of the tangent distribution}

The aim of this section is to show that the tangent distribution $T_{\mu}$ associated with a given Radon measure $\mu$ on $\mathbb{R}^{n}$ admits a 'geometric' characterisation in terms of the velocity of test plans, somehow refining Theorem 3.16. More precisely, we will prove that there exists a sequence $\left(\boldsymbol{\pi}_{i}\right)_{i}$ of test plans on $\left(\mathbb{R}^{n}, \mathrm{~d}_{\text {Eucl }}, \mu\right)$ having the following property: $T_{\mu}$ is obtained as the closure of the velocities of the plans $\pi_{i}$ at time 0 , in a suitable sense; see Theorem 4.16 for the correct statement. In order to achieve this goal, a key tool is given by the notion of test plan representing a gradient, which has been defined and proven to exist (in high generality) by N. Gigli in [23].

\subsubsection{Reminder on test plans representing a gradient}

First of all, let us report the notion of test plan representing the gradient of a Sobolev function; recall the definition (2.5) of $\mathrm{KE}_{t}$.

Definition 4.11 (Test plan representing a gradient [23]) Let $(X, \mathrm{~d}, \mu)$ be a metric measure space. Let $f \in W^{1,2}(X, \mu)$ be given. Then, a test plan $\pi$ on $(X, \mathrm{~d}, \mu)$ is said to represent the gradient of the function $f$ provided it satisfies the following property:

$$
\lim _{t \searrow 0} \frac{f \mathrm{oe}_{t}-f \mathrm{oe}_{0}}{\mathrm{KE}_{t}}=\lim _{t \searrow 0} \frac{\mathrm{KE}_{t}}{t}=\left|D_{\mu} f\right| \circ \mathrm{e}_{0}, \quad \text { strongly in } L^{2}(\boldsymbol{\pi}) .
$$


Test plans representing a gradient exist under mild assumptions, as the next result shows.

Theorem 4.12 (Existence of test plans representing a gradient [23]) Let (X, d, $\mu$ ) be a metric measure space. Let $v$ be a Borel probability measure on $(X, \mathrm{~d})$ such that $\int \mathrm{d}^{2}(\cdot, \bar{x}) \mathrm{d} v<+\infty$ for every $\bar{x} \in X$, and $v \leq C \mu$ for some constant $C>0$. Let $f \in W^{1,2}(X, \mu)$ be given. Then, there exists a test plan $\pi$ on $(X, d, \mu)$ that represents the gradient of $f$ and satisfies $\left(\mathrm{e}_{0}\right)_{*} \pi=v$.

In lack of an appropriate reference, we provide a quick proof of the following elementary continuity result. To do so, we use the well-known density of $\operatorname{LIP}_{c}\left(\mathbb{R}^{n}, \mathbb{R}^{n}\right)$ in $L^{2}\left(\mathbb{R}^{n}, \mathbb{R}^{n} ; \mu\right)$.

Lemma 4.13 Let $\mu \geq 0$ be a Radon measure on $\mathbb{R}^{n}$. Let $\pi$ be a test plan on $\left(\mathbb{R}^{n}, \mathrm{~d}_{\mathrm{Eucl}}, \mu\right)$. Then, for every $\underline{v} \in L^{2}\left(\mathbb{R}^{n}, \mathbb{R}^{n} ; \mu\right)$ it holds that

$$
[0,1] \ni t \longmapsto \underline{v o e}_{t} \in \mathbb{B}_{\pi} \text { is a continuous curve. }
$$

Proof Fix any $\underline{v} \in L^{2}\left(\mathbb{R}^{n}, \mathbb{R}^{n} ; \mu\right)$. Choose compactly supported Lipschitz maps $\underline{v}_{i}: \mathbb{R}^{n} \rightarrow \mathbb{R}^{n}$ such that $\underline{v}_{i} \rightarrow \underline{v}$ in $L^{2}\left(\mathbb{R}^{n}, \mathbb{R}^{n} ; \mu\right)$. Given any $t \in[0,1]$, we have $\lim _{s \rightarrow t} \int\left|\underline{v}_{i} \circ \mathrm{e}_{s}-\underline{v}_{i} \circ \mathrm{oe}_{t}\right|^{2} \mathrm{~d} \pi \stackrel{-i}{=} 0$ by dominated convergence theorem, so $[0,1] \ni t \mapsto \underline{v}_{i} \circ \mathrm{e}_{t} \in \mathbb{B}_{\pi}$ is continuous. Moreover, the curves $t \mapsto \underline{v}_{i} \circ \mathrm{e}_{t}$ uniformly converge to $t \mapsto \underline{v o e}_{t}$ as $i \rightarrow \infty$. Indeed, it holds that

$$
\sup _{t \in[0,1]} \int\left|\underline{v}_{i} \circ \mathrm{e}_{t}-\underline{v} \underline{\mathrm{oe}}_{t}\right|^{2} \mathrm{~d} \boldsymbol{\pi}=\sup _{t \in[0,1]} \int\left|\underline{v}_{i}-\underline{v}\right|^{2} \circ \mathrm{e}_{t} \mathrm{~d} \boldsymbol{\pi} \leq \operatorname{Comp}(\boldsymbol{\pi}) \int\left|\underline{v}_{i}-\underline{v}\right|^{2} \mathrm{~d} \mu .
$$

Therefore, the curve $[0,1] \ni t \mapsto \underline{v o e}_{t} \in \mathbb{B}_{\pi}$ is continuous as well, as required.

As one might expect, if a test plan $\pi$ represents the gradient of a Sobolev function $f$, then for any other Sobolev function $g$ we have, roughly speaking, that the derivative at $t=0$ of the map $t \mapsto g \circ \mathrm{e}_{t} \in L^{1}(\boldsymbol{\pi})$ coincides with the scalar product $\left\langle\nabla_{\mu} g, \nabla_{\mu} f\right\rangle \circ \mathrm{e}_{0}$. This claim is made precise by the ensuing result, which has been proven in [35, Corollary 2.4].

Proposition 4.14 Let $(X, \mathrm{~d}, \mu)$ be an infinitesimally Hilbertian space. Let $f \in W^{1,2}(X, \mu)$ be given. Let $\pi$ be a test plan on $(X, \mathrm{~d}, \mu)$ that represents the gradient of $f$. Then, for every function $g \in W^{1,2}(X, \mu)$ it holds that

$$
\frac{g \circ \mathrm{e}_{t}-g \circ \mathrm{e}_{0}}{t} \rightarrow\left\langle\nabla_{\mu} g, \nabla_{\mu} f\right\rangle \circ \mathrm{e}_{0}, \quad \text { weakly in } L^{1}(\pi) \text { as } t \searrow 0 .
$$

\subsubsection{Geometric characterisation of the tangent fibres}

In the setting of weighted Euclidean spaces, we have that test plans representing a gradient admit a 'concrete' derivative at $t=0$ :

Theorem 4.15 (Initial velocity of test plans representing a gradient) Let $\mu \geq 0$ be $a$ Radon measure on $\mathbb{R}^{n}$. Let $f \in W^{1,2}\left(\mathbb{R}^{n}, \mu\right)$ be given. Let $\pi$ be a test plan on $\left(\mathbb{R}^{n}, \mathrm{~d}_{\mathrm{Eucl}}, \mu\right)$ that represents the gradient of $f$. Then, it holds that 


$$
\exists \mathrm{D}_{\pi}:=\lim _{t \searrow 0} \frac{\mathrm{e}_{t}-\mathrm{e}_{0}}{t}=\iota_{\mu}\left(\nabla_{\mu} f\right) \circ \mathrm{e}_{0}, \quad \text { strongly in } \mathbb{B}_{\pi} .
$$

Proof Fix any sequence $t_{i} \searrow 0$. Observe that for every $t \in(0,1)$ we have that

$$
\left|\frac{\mathrm{e}_{t}-\mathrm{e}_{0}}{t}\right|(\gamma) \leq f_{0}^{t}\left|\dot{\gamma}_{s}\right| \mathrm{d} s \leq\left(f_{0}^{t}\left|\dot{\gamma}_{s}\right|^{2} \mathrm{~d} s\right)^{1 / 2}=\frac{\mathrm{KE}_{t}(\gamma)}{t}, \quad \text { for } \pi \text {-a.e. } \gamma \text {. }
$$

Since $\left(\mathrm{KE}_{t_{i}} / t_{i}\right)_{i}$ is convergent in $L^{2}(\pi)$, we deduce that $\left(\left(\mathrm{e}_{t_{i}}-\mathrm{e}_{0}\right) / t_{i}\right)_{i}$ is bounded in $\mathbb{B}_{\pi}$, thus accordingly (up to a not relabelled subsequence) it holds that $\left(\mathrm{e}_{t_{i}}-\mathrm{e}_{0}\right) / t_{i} \rightarrow \ell$ weakly in $\mathbb{B}_{\pi}$ for some $\ell \in \mathbb{B}_{\pi}$. Given $\underline{v} \in L^{2}\left(\mathbb{R}^{n}, \mathbb{R}^{n} ; \mu\right)$ and $E \subseteq C\left([0,1], \mathbb{R}^{n}\right)$ Borel, we claim that

$$
\int_{E}\left(\underline{v o e}_{0}\right) \cdot \ell \mathrm{d} \pi=\lim _{i \rightarrow \infty} \int_{E} f_{0}^{t_{i}}\left(\underline{v o e}_{t}\right) \cdot \operatorname{Der}_{t} \mathrm{~d} t \mathrm{~d} \pi
$$

In order to prove it, observe that

$$
\int_{E}\left(\underline{v o e}_{0}\right) \cdot \ell \mathrm{d} \pi=\lim _{i \rightarrow \infty} \int_{E}\left(\underline{v^{\circ}} \mathrm{e}_{0}\right) \cdot \frac{\mathrm{e}_{t_{i}}-\mathrm{e}_{0}}{t_{i}} \mathrm{~d} \pi \stackrel{(1.20)}{=} \lim _{i \rightarrow \infty} \int_{E} f_{0}^{t_{i}}\left(\underline{v_{0}} \mathrm{e}_{0}\right) \cdot \operatorname{Der}_{t} \mathrm{~d} t \mathrm{~d} \pi
$$

and that by exploiting Lemma 4.13 we obtain that

$$
\begin{aligned}
& \underset{i \rightarrow \infty}{\varlimsup_{i \rightarrow \infty}}\left|\int_{E} f_{0}^{t_{i}}\left(\underline{v o e}_{t}\right) \cdot \operatorname{Der}_{t} \mathrm{~d} t \mathrm{~d} \pi-\int_{E} f_{0}^{t_{i}}\left(\underline{v o e}_{0}\right) \cdot \operatorname{Der}_{t} \mathrm{~d} t \mathrm{~d} \pi\right| \\
\leq & \varlimsup_{i \rightarrow \infty} \int_{E} f_{0}^{t_{i}}\left|\underline{v o e}_{t}-\underline{v}^{\circ} \mathrm{e}_{0}\right|\left|\operatorname{Der}_{t}\right| \mathrm{d} t \mathrm{~d} \pi \\
\leq & \lim _{i \rightarrow \infty}\left(f_{0}^{t_{i}}\left\|\underline{v o e}_{t}-\underline{v o e}_{0}\right\|_{\mathbb{B}_{\pi}}^{2} \mathrm{~d} t\right)^{1 / 2}\left(\int \frac{\mathrm{KE}_{t_{i}}^{2}}{t_{i}^{2}} \mathrm{~d} \pi\right)^{1 / 2}=0 .
\end{aligned}
$$

Since one has $\operatorname{Der}_{t}(\gamma) \in T_{\mu}\left(\gamma_{t}\right)$ for $\left(\boldsymbol{\pi} \otimes \mathcal{L}_{1}\right)$-a.e. $(\gamma, t)$ by Lemma 3.6, we deduce from (4.8) that $\int_{E}\left(\underline{v o e}_{0}\right) \cdot \ell \mathrm{d} \pi=0$ for every $\underline{v} \in \Gamma\left(T_{\mu}^{\perp}\right)$ and $E \subseteq C\left([0,1], \mathbb{R}^{n}\right)$ Borel, thus accordingly

$$
\ell(\gamma) \in T_{\mu}\left(\gamma_{0}\right), \quad \text { for } \pi \text {-a.e. } \gamma \text {. }
$$

Also, given $g \in C_{c}^{\infty}\left(\mathbb{R}^{n}\right)$ and $E \subseteq C\left([0,1], \mathbb{R}^{n}\right)$ Borel, we know from Proposition 4.14 that

$$
\begin{aligned}
& \int_{E}\left(l_{\mu}\left(\nabla_{\mu} g\right) \circ \mathrm{e}_{0}\right) \cdot\left(l_{\mu}\left(\nabla_{\mu} f\right) \circ \mathrm{e}_{0}\right) \mathrm{d} \pi \\
= & \int_{E}\left\langle\nabla_{\mu} g, \nabla_{\mu} f\right\rangle \circ \mathrm{e}_{0} \mathrm{~d} \pi=\lim _{i \rightarrow \infty} \int_{E} \frac{g \circ \mathrm{e}_{t_{i}}-g \circ \mathrm{e}_{0}}{t_{i}} \mathrm{~d} \pi \\
= & \lim _{i \rightarrow \infty} \int_{E} f_{0}^{t_{i}} \frac{\mathrm{d}}{\mathrm{d} t} g\left(\gamma_{t}\right) \mathrm{d} t \mathrm{~d} \pi(\gamma)=\lim _{i \rightarrow \infty} \int_{E} f_{0}^{t_{i}}\left(\nabla g \circ \mathrm{e}_{t}\right) \cdot \operatorname{Der}_{t} \mathrm{~d} t \mathrm{~d} \pi \\
= & \lim _{i \rightarrow \infty} \int_{E} f_{0}^{t_{i}}\left(\mathrm{pr}_{T_{\mu}}(\nabla g) \circ \mathrm{e}_{t}\right) \cdot \operatorname{Der}_{t} \mathrm{~d} t \mathrm{~d} \pi \\
\stackrel{(2.15)}{=} & \lim _{i \rightarrow \infty} \int_{E} f_{0}^{t_{i}}\left(l_{\mu}\left(\nabla_{\mu} g\right) \circ \mathrm{e}_{t}\right) \cdot \operatorname{Der}_{t} \mathrm{~d} t \mathrm{~d} \boldsymbol{\pi} \stackrel{(3.8)}{=} \int_{E}\left(l_{\mu}\left(\nabla_{\mu} g\right) \circ \mathrm{e}_{0}\right) \cdot \ell \mathrm{d} \pi,
\end{aligned}
$$


whence it follows that $\left(\imath_{\mu}\left(\nabla_{\mu} g\right) \circ \mathrm{e}_{0}\right) \cdot\left(\ell-\iota_{\mu}\left(\nabla_{\mu} f\right) \circ \mathrm{e}_{0}\right)=0$ holds $\pi$-a.e.. By using (4.9) and the arbitrariness of $g \in C_{c}^{\infty}\left(\mathbb{R}^{n}\right)$, we get $\ell=\iota_{\mu}\left(\nabla_{\mu} f\right) \circ \mathrm{e}_{0}$. Being the limit $\ell$ independent of the sequence $\left(t_{i}\right)_{i}$, we deduce that

$$
\frac{\mathrm{e}_{t}-\mathrm{e}_{0}}{t} \rightarrow l_{\mu}\left(\nabla_{\mu} f\right) \circ \mathrm{e}_{0}, \quad \text { weakly in } \mathbb{B}_{\pi} \text { as } t \searrow 0 .
$$

Finally, let us observe that

$$
\begin{aligned}
\int\left|D_{\mu} f\right|^{2} \circ \mathrm{e}_{0} \mathrm{~d} \boldsymbol{\pi} & =\int\left|l_{\mu}\left(\nabla_{\mu} f\right)\right|^{2} \circ \mathrm{e}_{0} \mathrm{~d} \boldsymbol{\pi} \stackrel{(3.10)}{\leq} \frac{\lim }{t \searrow 0} \int\left|\frac{\mathrm{e}_{t}-\mathrm{e}_{0}}{t}\right|^{2} \mathrm{~d} \boldsymbol{\pi} \leq \varlimsup_{t \searrow 0} \int\left|\frac{\mathrm{e}_{t}-\mathrm{e}_{0}}{t}\right|^{2} \mathrm{~d} \boldsymbol{\pi} \\
& \stackrel{(3.7)}{\leq} \lim _{t \searrow 0} \int \frac{\mathrm{KE}_{t}^{2}}{t^{2}} \mathrm{~d} \boldsymbol{\pi}=\int\left|D_{\mu} f\right|^{2} \circ \mathrm{e}_{0} \mathrm{~d} \boldsymbol{\pi} .
\end{aligned}
$$

This shows that $\int\left|l_{\mu}\left(\nabla_{\mu} f\right)\right|^{2}$ oe $_{0} \mathrm{~d} \pi=\lim _{t \backslash 0} \int\left|\frac{\mathrm{e}_{t}-\mathrm{e}_{0}}{t}\right|^{2} \mathrm{~d} \pi$, which together with (4.10) grant that $\frac{\mathrm{e}_{t}-\mathrm{e}_{0}}{t} \rightarrow \iota_{\mu}\left(\nabla_{\mu} f\right) \circ \mathrm{e}_{0}$ strongly in $\mathbb{B}_{\pi}$ as $t \searrow 0$, thus proving the statement.

By building upon Theorem 4.15, we can eventually prove the main result of this section.

Theorem 4.16 (Geometric characterisation of the tangent fibres) Let $\mu \geq 0$ be a Radon measure on $\mathbb{R}^{n}$. Then, there exists a sequence $\left(\pi_{i}\right)_{i}$ of test plans on $\left(\mathbb{R}^{n}, \mathrm{~d}_{\text {Eucl }}, \mu\right)$ such that the limits $\mathrm{D}_{\pi_{i}}$ exist as in (4.6), the property $\mu \ll\left(\mathrm{e}_{0}\right)_{*} \pi_{i} \ll \mu$ holds for every $i \in \mathbb{N}$, and

$$
T_{\mu}(x)=\mathrm{cl}\left\{\operatorname{Im}_{\mathrm{e}_{0}, \pi_{i}}\left(\mathrm{D}_{\pi_{i}}\right)(x) \mid i \in \mathbb{N}\right\}, \quad \text { for } \mu \text {-a.e. } x \in \mathbb{R}^{n},
$$

where the essential image $\operatorname{Im}_{\mathrm{e}_{0}, \pi_{i}}\left(\mathrm{D}_{\pi_{i}}\right): C\left([0,1], \mathbb{R}^{n}\right) \rightarrow \mathbb{R}^{n}$ of $\mathrm{D}_{\pi_{i}}$ under $\mathrm{e}_{0}$ is defined as

$$
\operatorname{Im}_{\mathrm{e}_{0}, \boldsymbol{\pi}_{i}}\left(\mathrm{D}_{\boldsymbol{\pi}_{i}}\right):=\frac{\mathrm{d}\left(\mathrm{e}_{0}\right)_{*}\left(\mathrm{D}_{\boldsymbol{\pi}_{i}} \boldsymbol{\pi}_{i}\right)}{\mathrm{d}\left(\mathrm{e}_{0}\right)_{*} \boldsymbol{\pi}_{i}}, \quad \text { for every } i \in \mathbb{N} \text {. }
$$

Proof Given that $C_{c}^{\infty}\left(\mathbb{R}^{n}\right)$ is strongly dense in $W^{1,2}\left(\mathbb{R}^{n}, \mu\right)$ by Corollary 2.19 , we can find a countable $\mathbb{Q}$-linear subspace $\left(f_{i}\right)_{i}$ of $C_{c}^{\infty}\left(\mathbb{R}^{n}\right)$ that is dense in $W^{1,2}\left(\mathbb{R}^{n}, \mu\right)$. In particular, the family $\mathscr{V}:=\left\{\sum_{j=1}^{k} g_{j} \nabla_{\mu} f_{i_{j}}: k \in \mathbb{N},\left(g_{j}\right)_{j=1}^{k} \subseteq L^{\infty}(\mu),\left(i_{j}\right)_{j=1}^{k} \subseteq \mathbb{N}\right\}$ is dense in $L_{\mu}^{2}\left(T \mathbb{R}^{n}\right)$, thus the linear space $\iota_{\mu}(\mathscr{V})$ is dense in $\Gamma\left(T_{\mu}\right)$. By using Lemma 2.24, we can deduce that

$$
T_{\mu}(x)=\operatorname{cl}\left\{l_{\mu}\left(\nabla_{\mu} f_{i}\right)(x) \mid i \in \mathbb{N}\right\}, \quad \text { for } \mu \text {-a.e. } x \in \mathbb{R}^{n} .
$$

It is straightforward to check that one can find a Borel probability measure $v$ on $\mathbb{R}^{n}$ such that $\int|x|^{2} \mathrm{~d} \nu(x)<+\infty$ and $\mu \ll \nu \leq C \mu$ for some $C>0$. Given any $i \in \mathbb{N}$, we know from Theorem 4.12 that there exists a test plan $\pi_{i}$ on $\left(\mathbb{R}^{n}, \mathrm{~d}_{\text {Eucl }}, \mu\right)$ representing the gradient of $f_{i}$ and satisfying $\left(\mathrm{e}_{0}\right)_{*} \boldsymbol{\pi}_{i}=v$. Theorem 4.15 grants that $\mathrm{D}_{\boldsymbol{\pi}_{i}}$ exists as in (4.6). Also, it holds

$$
\begin{aligned}
\operatorname{Im}_{\mathrm{e}_{0}, \pi_{i}}\left(\mathrm{D}_{\boldsymbol{\pi}_{i}}\right) & =\operatorname{Im}_{\mathrm{e}_{0}, \pi_{i}}\left(\iota_{\mu}\left(\nabla_{\mu} f_{i}\right) \circ \mathrm{e}_{0}\right)=\frac{\mathrm{d}\left(\mathrm{e}_{0}\right)_{*}\left(\iota_{\mu}\left(\nabla_{\mu} f_{i}\right) \circ \mathrm{e}_{0} \pi_{i}\right)}{\mathrm{d} v}=\frac{\mathrm{d}\left(\iota_{\mu}\left(\nabla_{\mu} f_{i}\right) v\right)}{\mathrm{d} v} \\
& =l_{\mu}\left(\nabla_{\mu} f_{i}\right) .
\end{aligned}
$$

By taking (4.12) into account, we eventually obtain (4.11), as desired. 


\subsection{Tensorisation of the Cheeger energy on weighted Euclidean spaces}

In the framework of Sobolev calculus on metric measure spaces, a surprisingly difficult problem is the following: given two metric measure spaces $\left(X, \mathrm{~d}_{X}, \mu\right)$ and $\left(Y, \mathrm{~d}_{Y}, v\right)$, is the Sobolev space on the product space $\left(X \times Y, \mathrm{~d}_{X \times Y}, \mu \otimes v\right)$ the tensorisation of $W^{1,2}(X, \mu)$ and $W^{1,2}(Y, v)$ ?

The precise statement would read as follows: given any function $f \in W^{1,2}(X \times Y, \mu \otimes v)$, it holds for $(\mu \otimes v)$-a.e. $(x, y) \in X \times Y$ that $f^{(y)} \in W^{1,2}(X, \mu), f_{(x)} \in W^{1,2}(Y, v)$, and

$$
\left|D_{\mu \otimes v} f\right|^{2}(x, y)=\left|D_{\mu} f^{(y)}\right|^{2}(x)+\left|D_{v} f_{(x)}\right|^{2}(y),
$$

where we set $f^{(y)}(x)=f_{(x)}(y):=f(x, y)$. (Here, Fubini theorem plays a role.)

A positive answer to the above question is known only in some particular circumstances. About the spaces having such tensorisation property, this is the current state of the art:

(a) Ambrosio et al. [7] proved that $\operatorname{RCD}(K, \infty)$ spaces, for any given $K \in \mathbb{R}$, have the tensorisation property.

(b) Ambrosio et al. [8] proved the tensorisation property on doubling metric measure spaces supporting a weak $(1,2)$-Poincaré inequality.

(c) Gigli and Han [26] showed that the Sobolev space tensorises as soon as one of the two factors is a closed real interval $I \subseteq \mathbb{R}$.

To the best of our knowledge, these are all the cases that have been studied so far. The aim of this section is to prove that weighted Euclidean spaces have the tensorisation property (cf. Theorem 4.21), and we do so by first showing that the fibres of the tangent distribution 'tensorise' as well (cf. Proposition 4.19). Notice that the family of all weighted Euclidean spaces is not contained in any of the classes of spaces described in items a), b), and c) above.

\subsubsection{Test plans on product spaces}

Let $\left(X, \mathrm{~d}_{X}, \mu\right),\left(Y, \mathrm{~d}_{Y}, \nu\right)$ be two given metric measure spaces. The Cartesian product $X \times Y$ will be implicitly endowed with the product distance

$$
\mathrm{d}_{X \times Y}\left((x, y),\left(x^{\prime}, y^{\prime}\right)\right):=\sqrt{\mathrm{d}_{X}\left(x, x^{\prime}\right)^{2}+\mathrm{d}_{Y}\left(y, y^{\prime}\right)^{2}}, \quad \text { for every }(x, y),\left(x^{\prime}, y^{\prime}\right) \in X \times Y,
$$

and the product measure $\mu \otimes v$. We denote by $p^{X}: X \times Y \rightarrow X$ and $p^{Y}: X \times Y \rightarrow Y$ the canonical projection maps $p^{X}(x, y):=x$ and $p^{Y}(x, y):=y$. They induce the 1-Lipschitz maps

$$
\begin{array}{ll}
p^{X}: C([0,1], X \times Y) \longrightarrow C([0,1], X), & \boldsymbol{p}^{X}(\gamma):=p^{X} \circ \gamma, \\
\boldsymbol{p}^{Y}: C([0,1], X \times Y) \longrightarrow C([0,1], Y), & \boldsymbol{p}^{Y}(\gamma):=p^{Y} \circ \gamma .
\end{array}
$$

It can be readily checked that

$$
\begin{aligned}
& \boldsymbol{p}^{X}\left(A C^{2}([0,1], X \times Y)\right) \subseteq A C^{2}([0,1], X), \\
& \boldsymbol{p}^{Y}\left(A C^{2}([0,1], X \times Y)\right) \subseteq A C^{2}([0,1], Y) .
\end{aligned}
$$


Moreover, let us consider the joint mapping

$$
\begin{aligned}
\left(\boldsymbol{p}^{X}, \boldsymbol{p}^{Y}\right): C([0,1], X \times Y) & \longrightarrow C([0,1], X) \times C([0,1], Y), \\
\gamma & \longmapsto\left(\boldsymbol{p}^{X}(\gamma), \boldsymbol{p}^{Y}(\gamma)\right) .
\end{aligned}
$$

It turns out that $\left(\boldsymbol{p}^{X}, \boldsymbol{p}^{Y}\right)$ is a $\sqrt{2}$-Lipschitz bijection whose inverse is 1-Lipschitz. Also,

$$
\left(\boldsymbol{p}^{X}, \boldsymbol{p}^{Y}\right)\left(A C^{2}([0,1], X \times Y)\right)=A C^{2}([0,1], X) \times A C^{2}([0,1], Y) .
$$

More precisely, given any curve $\gamma=\left(\gamma^{X}, \gamma^{Y}\right) \in A C^{2}([0,1], X \times Y)$, it holds that

$$
\left|\dot{\gamma}_{t}\right|^{2}=\left|\dot{\gamma}_{t}^{X}\right|^{2}+\left|\dot{\gamma}_{t}^{Y}\right|^{2}, \quad \text { for } \mathcal{L}_{1} \text {-a.e. } t \in[0,1] \text {. }
$$

For completeness, we report below the elementary proofs of the following two technical results:

Lemma 4.17 Let $\left(X, \mathrm{~d}_{X}, \mu\right),\left(Y, \mathrm{~d}_{Y}, v\right)$ be metric measure spaces such that $\mu, v$ are finite Borel measures. Let $\boldsymbol{\pi}$ be a given test plan on $\left(X \times Y, \mathrm{~d}_{X \times Y}, \mu \otimes v\right)$. Then $\boldsymbol{\pi}_{X}:=\boldsymbol{p}_{*}^{X} \boldsymbol{\pi}$ is a test plan on $\left(X, \mathrm{~d}_{X}, \mu\right)$ and $\boldsymbol{\pi}_{Y}:=\boldsymbol{p}_{*}^{Y} \boldsymbol{\pi}$ is a test plan on $\left(Y, \mathrm{~d}_{Y}, v\right)$. Moreover, it holds that

$$
\operatorname{Comp}\left(\boldsymbol{\pi}_{X}\right) \leq \operatorname{Comp}(\boldsymbol{\pi}) v(Y), \quad \operatorname{Comp}\left(\boldsymbol{\pi}_{Y}\right) \leq \operatorname{Comp}(\boldsymbol{\pi}) \mu(X) .
$$

Proof By symmetry, it suffices to prove the statement just for $\pi_{X}$. Since $\pi$ is concentrated on $A C^{2}([0,1], X \times Y)$, we have that $\pi_{X}$ is concentrated on $A C^{2}([0,1], X)$. Moreover, for any curve $\gamma=\left(\gamma^{X}, \gamma^{Y}\right) \in A C^{2}([0,1], X \times Y)$ it holds $\left|\dot{\gamma}_{t}^{X}\right| \leq\left|\dot{\gamma}_{t}\right|$ for $\mathcal{L}_{1}$-a.e. $t \in[0,1]$, whence

$$
\iint_{0}^{1}\left|\dot{\gamma}_{t}^{X}\right|^{2} \mathrm{~d} t \mathrm{~d} \pi_{X}\left(\gamma^{X}\right)=\iint_{0}^{1}\left|\dot{\gamma}_{t}^{X}\right|^{2} \mathrm{~d} t \mathrm{~d} \boldsymbol{\pi}\left(\gamma^{X}, \gamma^{Y}\right) \leq \iint_{0}^{1}\left|\dot{\gamma}_{t}\right|^{2} \mathrm{~d} t \mathrm{~d} \boldsymbol{\pi}(\gamma)<+\infty .
$$

Finally, for any Borel set $A \subseteq X$ we have that

$$
\begin{aligned}
\left(\mathrm{e}_{t}^{X}\right)_{*} \pi_{X}(A)=\pi_{X}\left(\left(\mathrm{e}_{t}^{X}\right)^{-1}(A)\right) & =\pi\left(\left(\mathrm{e}_{t}^{X \times Y}\right)^{-1}(A \times Y)\right)=\left(\mathrm{e}_{t}^{X \times Y}\right)_{*} \pi(A \times Y) \\
& \leq \operatorname{Comp}(\pi)(\mu \otimes v)(A \times Y)=\operatorname{Comp}(\pi) v(Y) \mu(A),
\end{aligned}
$$

for all $t \in[0,1]$. Hence, $\boldsymbol{\pi}_{X}$ is a test plan on $\left(X, \mathrm{~d}_{X}, \mu\right)$ and $\operatorname{Comp}\left(\boldsymbol{\pi}_{X}\right) \leq \operatorname{Comp}(\boldsymbol{\pi}) v(Y)$.

Lemma 4.18 Let $\left(X, \mathrm{~d}_{X}, \mu\right),\left(Y, \mathrm{~d}_{Y}, v\right)$ be metric measure spaces. Let $\pi_{X}$ and $\pi_{Y}$ be test plans on $\left(X, \mathrm{~d}_{X}, \mu\right)$ and $\left(Y, \mathrm{~d}_{Y}, v\right)$, respectively. Then $\boldsymbol{\pi}:=\left(\boldsymbol{p}^{X}, \boldsymbol{p}^{Y}\right)_{*}^{-1}\left(\boldsymbol{\pi}_{X} \otimes \boldsymbol{\pi}_{Y}\right)$ is a test plan on $\left(X \times Y, \mathrm{~d}_{X \times Y}, \mu \otimes v\right)$. Moreover, it holds that $\operatorname{Comp}(\boldsymbol{\pi}) \leq \operatorname{Comp}\left(\boldsymbol{\pi}_{X}\right) \operatorname{Comp}\left(\boldsymbol{\pi}_{Y}\right)$.

Proof We know from (4.14) that $\pi$ is concentrated on $A C^{2}([0,1], X \times Y)$, while (4.15) yields

$$
\iint_{0}^{1}\left|\dot{\gamma}_{t}\right|^{2} \mathrm{~d} t \mathrm{~d} \boldsymbol{\pi}(\gamma)=\iint_{0}^{1}\left|\dot{\gamma}_{t}^{X}\right|^{2} \mathrm{~d} t \mathrm{~d} \pi_{X}\left(\gamma^{X}\right)+\iint_{0}^{1}\left|\dot{\gamma}_{t}^{Y}\right|^{2} \mathrm{~d} t \mathrm{~d} \pi_{Y}\left(\gamma^{Y}\right)<+\infty .
$$

Moreover, given any non-negative Borel function $f$ on $X \times Y$, for every $t \in[0,1]$ it holds that 


$$
\begin{aligned}
\int f \mathrm{~d}\left(\mathrm{e}_{t}^{X \times Y}\right)_{*} \boldsymbol{\pi} & =\int f\left(\gamma_{t}\right) \mathrm{d} \boldsymbol{\pi}(\gamma)=\iint f\left(\gamma_{t}^{X}, \gamma_{t}^{Y}\right) \mathrm{d} \boldsymbol{\pi}_{X}\left(\gamma^{X}\right) \mathrm{d} \boldsymbol{\pi}_{Y}\left(\gamma^{Y}\right) \\
& =\iint f(x, y) \mathrm{d}\left(\mathrm{e}_{t}^{X}\right)_{*} \boldsymbol{\pi}_{X}(x) \mathrm{d}\left(\mathrm{e}_{t}^{Y}\right)_{*} \boldsymbol{\pi}_{Y}(y) \\
& \leq \operatorname{Comp}\left(\boldsymbol{\pi}_{X}\right) \operatorname{Comp}\left(\boldsymbol{\pi}_{Y}\right) \iint f(x, y) \mathrm{d} \mu(x) \mathrm{d} v(y) \\
& =\operatorname{Comp}\left(\boldsymbol{\pi}_{X}\right) \operatorname{Comp}\left(\boldsymbol{\pi}_{Y}\right) \int f \mathrm{~d}(\mu \otimes v),
\end{aligned}
$$

whence $\left(\mathrm{e}_{t}^{X \times Y}\right)_{*} \pi \leq \operatorname{Comp}\left(\pi_{X}\right) \operatorname{Comp}\left(\pi_{Y}\right) \mu \otimes v$. This proves the statement.

\subsubsection{Tensorisation of the tangent distribution}

Let us denote by $p^{n}$ and $p^{m}$ the canonical projections of the product $\mathbb{R}^{n+m} \cong \mathbb{R}^{n} \times \mathbb{R}^{m}$ onto $\mathbb{R}^{n}$ and $\mathbb{R}^{m}$, respectively, instead of $p^{\mathbb{R}^{n}}$ and $p^{\mathbb{R}^{m}}$. Also, we define the embedding maps $\iota^{n}: \mathbb{R}^{n} \rightarrow \mathbb{R}^{n+m}$ and $\iota^{m}: \mathbb{R}^{m} \rightarrow \mathbb{R}^{n+m}$ as

$$
\begin{aligned}
& \iota^{n}(v):=(v, 0) \in \mathbb{R}^{n} \times \mathbb{R}^{m}, \quad \text { for every } v \in \mathbb{R}^{n}, \\
& l^{m}(w):=(0, w) \in \mathbb{R}^{n} \times \mathbb{R}^{m}, \quad \text { for every } w \in \mathbb{R}^{m} \text {. }
\end{aligned}
$$

Proposition 4.19 (Tangent distribution on the product space) Let $\mu$ and $v$ be finite Borel measures on $\mathbb{R}^{n}$ and $\mathbb{R}^{m}$, respectively. Then, it holds that

$$
T_{\mu \otimes \nu}(x, y)=\imath^{n}\left(T_{\mu}(x)\right) \oplus \imath^{m}\left(T_{\nu}(y)\right), \quad \text { for }(\mu \otimes v) \text {-a.e. }(x, y) \in \mathbb{R}^{n+m} .
$$

Proof Let us define $S(x, y):=\imath^{n}\left(T_{\mu}(x)\right) \oplus \imath^{m}\left(T_{\nu}(y)\right)$ for $(\mu \otimes v)$-a.e. $(x, y) \in \mathbb{R}^{n+m}$. It is straightforward to check that $S \in \mathscr{D}_{n+m}(\mu \otimes v)$. To prove the statement amounts to showing that $T_{\mu \otimes v}=S$. First, let us prove that $T_{\mu \otimes v} \leq S$. In light of item (i) of Theorem 3.16, this is equivalent to saying that for any test plan $\pi$ on $\left(\mathbb{R}^{n+m}, \mathrm{~d}_{\text {Eucl }}, \mu \otimes v\right)$ it holds that

$$
\dot{\gamma}_{t} \in S\left(\gamma_{t}\right), \quad \text { for }\left(\boldsymbol{\pi} \otimes \mathcal{L}_{1}\right) \text {-a.e. }(\gamma, t) \in A C^{2}\left([0,1], \mathbb{R}^{n+m}\right) \times[0,1] .
$$

Call $\boldsymbol{\pi}_{n}:=\boldsymbol{p}_{*}^{n} \boldsymbol{\pi}$ and $\boldsymbol{\pi}_{m}:=\boldsymbol{p}_{*}^{m} \boldsymbol{\pi}$. We know from Lemma 4.17 that $\boldsymbol{\pi}_{n}$ and $\boldsymbol{\pi}_{m}$ are test plans on $\left(\mathbb{R}^{n}, \mathrm{~d}_{\text {Eucl }}, \mu\right)$ and $\left(\mathbb{R}^{m}, \mathrm{~d}_{\text {Eucl }}, v\right)$, respectively. Hence, item (i) of Theorem 3.16 gives

$$
\begin{array}{cl}
\dot{\gamma}_{t}^{n} \in T_{\mu}\left(\gamma_{t}^{n}\right), & \text { for }\left(\boldsymbol{\pi}_{n} \otimes \mathcal{L}_{1}\right) \text {-a.e. }\left(\gamma^{n}, t\right) \in A C^{2}\left([0,1], \mathbb{R}^{n}\right) \times[0,1], \\
\dot{\gamma}_{t}^{m} \in T_{\nu}\left(\gamma_{t}^{m}\right), & \text { for }\left(\boldsymbol{\pi}_{m} \otimes \mathcal{L}_{1}\right) \text {-a.e. }\left(\gamma^{m}, t\right) \in A C^{2}\left([0,1], \mathbb{R}^{m}\right) \times[0,1],
\end{array}
$$

which can be equivalently restated as follows: for $\pi$-a.e. $\gamma=\left(\gamma^{n}, \gamma^{m}\right) \in A C^{2}\left([0,1], \mathbb{R}^{n+m}\right)$ it holds $\left(\dot{\gamma}_{t}^{n}, \dot{\gamma}_{t}^{m}\right) \in T_{\mu}\left(\gamma_{t}^{n}\right) \times T_{v}\left(\gamma_{t}^{m}\right)$ for $\mathcal{L}_{1^{-}}$a.e. $t \in[0,1]$. This proves (4.16), whence $T_{\mu \otimes v} \leq S$

In order to prove that $S \leq T_{\mu \otimes \nu}$, it is clearly sufficient to show that $T_{\mu}(x) \subseteq p^{n}\left(T_{\mu \otimes \nu}(x, y)\right)$ and $T_{v}(y) \subseteq p^{m}\left(T_{\mu \otimes v}(x, y)\right)$ hold for $(\mu \otimes v)$-a.e. $(x, y) \in \mathbb{R}^{n+m}$. Let us just prove the former inclusion, since the latter one can be obtained by an analogous argument. Trivially, we have that $p^{n}\left(T_{\mu \otimes v}(\cdot, y)\right) \in \mathscr{D}_{n}(\mu)$ for $v$-a.e. $y \in \mathbb{R}^{m}$. Now fix a test plan $\pi_{n}$ on $\left(\mathbb{R}^{n}, \mathrm{~d}_{\text {Eucl }}, \mu\right)$. We then define the measure $\boldsymbol{\pi}$ on $C\left([0,1], \mathbb{R}^{n+m}\right)$ as 


$$
\boldsymbol{\pi}:=\left(\boldsymbol{p}^{n}, \boldsymbol{p}^{m}\right)_{*}^{-1}\left(\boldsymbol{\pi}_{n} \otimes \mathrm{Const}_{*}^{m} \nu\right),
$$

where the map Const ${ }^{m}:=$ Const $^{\mathbb{R}^{m}}$ is defined as in Example 2.2. Lemma 4.18 grants that $\pi$ is a test plan on $\left(\mathbb{R}^{n+m}, \mathrm{~d}_{\mathrm{Eucl}}, \mu \otimes v\right)$, thus item (i) of Theorem 3.16 ensures that $\dot{\gamma}_{t} \in T_{\mu \otimes v}\left(\gamma_{t}\right)$ is satisfied for $\left(\boldsymbol{\pi} \otimes \mathcal{L}_{1}\right)$-a.e. $(\gamma, t) \in A C^{2}\left([0,1], \mathbb{R}^{n+m}\right) \times[0,1]$. This can be rewritten as

$$
\left(\dot{\gamma}_{t}^{n}, 0\right) \in T_{\mu \otimes v}\left(\gamma_{t}^{n}, y\right), \quad \text { for }\left(\pi_{n} \otimes \nu \otimes \mathcal{L}_{1}\right) \text {-a.e. }\left(\gamma^{n}, y, t\right) \in A C^{2}\left([0,1], \mathbb{R}^{n}\right) \times \mathbb{R}^{m} \times[0,1] .
$$

Therefore, by arbitrariness of $\pi_{n}$ we can finally conclude that $T_{\mu}(x) \subseteq p^{n}\left(T_{\mu \otimes \nu}(x, y)\right)$ holds for $(\mu \otimes v)$-a.e. $(x, y) \in \mathbb{R}^{n+m}$, whence the proof of the statement is complete.

Remark 4.20 Proposition 4.19 is claimed in [9, Remark 2.2(iv)]. Therein, the tangent distribution is defined in terms of the distributional divergence, an approach that is equivalent to ours in view of item (ii) of Theorem 3.16.

\subsubsection{Tensorisation of the Sobolev space}

We are in a position-by exploiting Propositions 3.19 and 4.19- to prove that weighted Euclidean spaces have the tensorisation property.

Given a Borel function $f: \mathbb{R}^{n+m} \rightarrow \mathbb{R}$, we define $f^{(y)}: \mathbb{R}^{n} \rightarrow \mathbb{R}$ and $f_{(x)}: \mathbb{R}^{m} \rightarrow \mathbb{R}$ as

$$
f^{(y)}(x)=f_{(x)}(y):=f(x, y), \quad \text { for every }(x, y) \in \mathbb{R}^{n+m} .
$$

Observe that $f^{(y)}$ and $f_{(x)}$ are Borel functions as well. Also, thanks to Fubini theorem, for every $f \in L^{2}(\mu \otimes v)$ we have $f^{(y)} \in L^{2}(\mu)$ for $v$-a.e. $y \in \mathbb{R}^{m}$ and $f_{(x)} \in L^{2}(v)$ for $\mu$-a.e. $x \in \mathbb{R}^{n}$.

Theorem 4.21 (Tensorisation of the Sobolev space on weighted $\mathbb{R}^{n}$ ) Let $\mu$ and $v$ be finite Borel measures on $\mathbb{R}^{n}$ and $\mathbb{R}^{m}$, respectively. Let $f \in W^{1,2}\left(\mathbb{R}^{n+m}, \mu \otimes v\right)$ be given. Then

$$
\begin{array}{ll}
f^{(y)} \in W^{1,2}\left(\mathbb{R}^{n}, \mu\right), & \text { for } v \text {-a.e. } y \in \mathbb{R}^{m}, \\
f_{(x)} \in W^{1,2}\left(\mathbb{R}^{m}, v\right), & \text { for } \mu \text {-a.e. } x \in \mathbb{R}^{n} .
\end{array}
$$

Moreover, it holds that

$$
\left|D_{\mu \otimes u} f\right|^{2}(x, y)=\left|D_{\mu} f^{(y)}\right|^{2}(x)+\left|D_{v} f_{(x)}\right|^{2}(y), \quad \text { for }(\mu \otimes v) \text {-a.e. }(x, y) \in \mathbb{R}^{n+m} .
$$

Proof First of all, we know from Proposition 3.19 that $G_{\mu \otimes v}(f) \neq \emptyset$ and $\iota_{\mu \otimes v}\left(\nabla_{\mu \otimes v} f\right)$ is the minimal $G_{\mu \otimes v}$-gradient of $f$. We can choose a sequence $\left(f_{i}\right)_{i} \subseteq C_{c}^{\infty}\left(\mathbb{R}^{n+m}\right)$ such that $f_{i} \rightarrow f$ in $L^{2}(\mu \otimes v)$ and $\nabla f_{i} \rightarrow l_{\mu \otimes \nu}\left(\nabla_{\mu \otimes v} f\right)$ in $L^{2}\left(\mathbb{R}^{n+m}, \mathbb{R}^{n+m} ; \mu \otimes v\right)$. Notice that $\left(f_{i}\right)^{(y)} \in C_{c}^{\infty}\left(\mathbb{R}^{n}\right)$ and $\left(f_{i}\right)_{(x)} \in C_{c}^{\infty}\left(\mathbb{R}^{m}\right)$ for every $i \in \mathbb{N}$ and $(x, y) \in \mathbb{R}^{n+m}$. Proposition 4.19 grants that

$$
\operatorname{pr}_{T_{\mu \otimes v}}\left(\nabla f_{i}\right)(x, y)=\left(\operatorname{pr}_{T_{\mu}}\left(\nabla\left(f_{i}\right)^{(y)}\right)(x), \operatorname{pr}_{T_{v}}\left(\nabla\left(f_{i}\right)_{(x)}\right)(y)\right)
$$

for $(\mu \otimes v)$-a.e. $(x, y) \in \mathbb{R}^{n+m}$. Recalling Proposition 3.19, we deduce from (4.19) that

$$
\left|D_{\mu \otimes v} f_{i}\right|^{2}(x, y)=\left|D_{\mu}\left(f_{i}\right)^{(y)}\right|^{2}(x)+\left|D_{\nu}\left(f_{i}\right)_{(x)}\right|^{2}(y), \quad \text { for }(\mu \otimes v) \text {-a.e. }(x, y) \in \mathbb{R}^{n+m} .
$$


Thanks to Fubini theorem, we have (up to a not relabelled subsequence) that

$$
\begin{array}{ll}
\left(f_{i}\right)^{(y)} \longrightarrow f^{(y)}, & \text { strongly in } L^{2}(\mu) \text { for } v \text {-a.e. } y \in \mathbb{R}^{m}, \\
\left(f_{i}\right)_{(x)} \longrightarrow f_{(x)}, & \text { strongly in } L^{2}(v) \text { for } \mu \text {-a.e. } x \in \mathbb{R}^{n} .
\end{array}
$$

Call $\underline{v}:=l_{\mu \otimes v}\left(\nabla_{\mu \otimes v} f\right) \in \Gamma\left(T_{\mu \otimes v}\right)$. We have that $\operatorname{pr}_{T_{\mu \otimes v}}\left(\nabla f_{i}\right) \rightarrow \underline{v}$ in $L^{2}\left(\mathbb{R}^{n+m}, \mathbb{R}^{n+m} ; \mu \otimes v\right)$, so that (up to passing to a further subsequence) it holds that

$$
\operatorname{pr}_{T_{\mu \otimes v}}\left(\nabla f_{i}\right)(x, y) \longrightarrow \underline{v}(x, y), \quad \text { for }(\mu \otimes v) \text {-a.e. }(x, y) \in \mathbb{R}^{n+m},
$$

thus in particular

$$
\left|D_{\mu \otimes v} f_{i}\right| \stackrel{(2.15)}{=}\left|\operatorname{pr}_{T_{\mu \otimes v}}\left(\nabla f_{i}\right)\right| \longrightarrow|\underline{v}|=\left|D_{\mu \otimes v} f\right|, \quad \text { in the }(\mu \otimes v) \text {-a.e. sense. }
$$

Set $\underline{v}^{(y)}(x):=p^{n}(\underline{v}(x, y)) \in T_{\mu}(x)$ and $\underline{v}_{(x)}(y):=p^{m}(\underline{v}(x, y)) \in T_{v}(y)$ for $(\mu \otimes v)$-a.e. $(x, y)$. Therefore, for $(\mu \bar{\otimes} v)$-a.e. $(x, y) \in \mathbb{R}^{n+\bar{m}}$ it holds that

$$
\begin{aligned}
& \iota_{\mu}\left(\nabla_{\mu}\left(f_{i}\right)^{(y)}\right) \stackrel{(2.15)}{=} \operatorname{pr}_{T_{\mu}}\left(\nabla\left(f_{i}\right)^{(y)}\right) \longrightarrow \underline{v}^{(y)}, \quad \text { strongly in } L^{2}\left(\mathbb{R}^{n}, \mathbb{R}^{n} ; \mu\right), \\
& \iota_{v}\left(\nabla_{v}\left(f_{i}\right)_{(x)}\right) \stackrel{(2.15)}{=} \operatorname{pr}_{T_{v}}\left(\nabla\left(f_{i}\right)_{(x)}\right) \longrightarrow \underline{v}_{(x)}, \quad \text { strongly in } L^{2}\left(\mathbb{R}^{m}, \mathbb{R}^{m} ; v\right) \text {. }
\end{aligned}
$$

This implies $\underline{v}^{(y)} \in \iota_{\mu}\left(L_{\mu}^{2}\left(T \mathbb{R}^{n}\right)\right)$ and $\underline{v}_{(x)} \in \iota_{\nu}\left(L_{\nu}^{2}\left(T \mathbb{R}^{m}\right)\right)$ for $(\mu \otimes v)$-a.e. $(x, y) \in \mathbb{R}^{n+m}$, and

$$
\begin{array}{ll}
\nabla_{\mu}\left(f_{i}\right)^{(y)} \longrightarrow l_{\mu}^{-1}\left(\underline{v}^{(y)}\right)=: v^{(y)}, & \text { strongly in } L_{\mu}^{2}\left(T \mathbb{R}^{n}\right), \\
\nabla_{\nu}\left(f_{i}\right)_{(x)} \longrightarrow l_{\nu}^{-1}\left(\underline{v}_{(x)}\right)=: v_{(x)}, & \text { strongly in } L_{\nu}^{2}\left(T \mathbb{R}^{m}\right) .
\end{array}
$$

Moreover, it follows from (4.19) and (4.22) that for $(\mu \otimes v)$-a.e. $(x, y) \in \mathbb{R}^{n+m}$ it holds that

$$
\begin{gathered}
\left|D_{\mu}\left(f_{i}\right)^{(y)}\right| \stackrel{(2.15)}{=}\left|\operatorname{pr}_{T_{\mu}}\left(\nabla\left(f_{i}\right)^{(y)}\right)\right| \longrightarrow\left|\underline{v}^{(y)}\right|=\left|v^{(y)}\right|, \quad \text { in the } \mu \text {-a.e. sense, } \\
\left|D_{\nu}\left(f_{i}\right)_{(x)}\right| \stackrel{(2.15)}{=}\left|\operatorname{pr}_{T_{v}}\left(\nabla\left(f_{i}\right)_{(x)}\right)\right| \longrightarrow\left|\underline{v}_{(x)}\right|=\left|v_{(x)}\right|, \quad \text { in the } v \text {-a.e. sense. }
\end{gathered}
$$

By applying Proposition 2.11, we deduce from (4.21) and (4.24) that (4.17) is satisfied, that $\nabla_{\mu} f^{(y)}=v^{(y)}$ for $v$-a.e. $y \in \mathbb{R}^{m}$, and that $\nabla_{\psi} f_{(x)}=v_{(x)}$ for $\mu$-a.e. $x \in \mathbb{R}^{n}$. Consequently, by letting $i \rightarrow \infty$ in (4.20) and using (4.23) and (4.25), wefinally conclude that (4.18) holds.

Remark 4.22 Proposition 4.19 and Theorem 4.21 are verified even when $\mu$ and $\nu$ are (not necessarily finite) Radon measures, by taking into account [23, Proposition 2.6], which says that the Sobolev space can be 'localised' in a suitable sense. We omit the details.

Acknowledgements We would like to thank Simone Di Marino for the many useful conversations about the results of this paper. All authors are partially supported by the Academy of Finland, Project 314789.

Funding Open access funding provided by University of Jyväskylä (JYU).

Open Access This article is licensed under a Creative Commons Attribution 4.0 International License, which permits use, sharing, adaptation, distribution and reproduction in any medium or format, as long as you give appropriate credit to the original author(s) and the source, provide a link to the Creative Commons 
licence, and indicate if changes were made. The images or other third party material in this article are included in the article's Creative Commons licence, unless indicated otherwise in a credit line to the material. If material is not included in the article's Creative Commons licence and your intended use is not permitted by statutory regulation or exceeds the permitted use, you will need to obtain permission directly from the copyright holder. To view a copy of this licence, visit http://creativecommons.org/licenses/by/4.0/.

\section{References}

1. Alberti, G., Marchese, A.: On the differentiability of Lipschitz functions with respect to measures in the Euclidean space. Geom. Funct. Anal. 26, 1-66 (2016)

2. Aliprantis, C., Border, K.: Infinite Dimensional Analysis: A Hitchhiker's Guide. Studies in Economic Theory. Springer, Berlin (1999)

3. Allard, W.K.: On the first variation of a varifold. Ann. Math. 95, 417-491 (1972)

4. Ambrosio, L., Gigli, N., Savaré, G.: Gradient Flows in Metric Spaces and in the Space of Probability Measures. Lectures in Mathematics, 2nd edn. ETH, Zürich (2008)

5. Ambrosio, L., Gigli, N., Savaré, G.: Density of Lipschitz functions and equivalence of weak gradients in metric measure spaces. Rev. Mat. Iberoam. 29, 969-996 (2013)

6. Ambrosio, L., Gigli, N., Savaré, G.: Calculus and heat flow in metric measure spaces and applications to spaces with Ricci bounds from below. Invent. Math. 195, 289-391 (2014)

7. Ambrosio, L., Gigli, N., Savaré, G.: Metric measure spaces with Riemannian Ricci curvature bounded from below. Duke Math. J. 163, 1405-1490 (2014)

8. Ambrosio, L., Pinamonti, A., Speight, G.: Tensorization of Cheeger energies, the space $H^{1,1}$ and the area formula for graphs. Adv. Math. 281, 1145-1177 (2014)

9. Bouchitté, G., Buttazzo, G., Seppecher, P.: Energies with respect to a measure and applications to low dimensional structures. Calc. Var. Partial Differ. Equ. 5, 37-54 (1997)

10. Bouchitté, G., Buttazzo, G., Seppecher, P.: Shape optimization solutions via Monge-Kantorovich equation. Comptes Rendus de l'Académie des Sci. Ser. I Math. 324, 1185-1191 (1997)

11. Bouchitté, G., Fragalà, I.: Variational theory of weak geometrical structures: the measure method and its applications. Prog. Nonlinear Differ. Equ. Appl. 51, 19-40 (2002)

12. Bouchitté, G., Fragalà, I.: Second-order energies on thin structures: variational theory and non-local effects. J. Funct. Anal. 204, 228-267 (2003)

13. Cheeger, J.: Differentiability of Lipschitz functions on metric measure spaces. Geom. Funct. Anal. 9, 428-517 (1999)

14. De Philippis, G., Marchese, A., Rindler, F.: On a conjecture of Cheeger. In: Measure Theory in Nonsmooth Spaces (2017)

15. De Philippis, G., Rindler, F.: On the structure of $A$-free measures and applications. Ann. Math. 184, 1017-1039 (2016)

16. Di Marino, S.: Recent advances on BV and Sobolev spaces in metric measure spaces. Ph.D. Thesis (2014)

17. Di Marino, S.: Sobolev and BV spaces on metric measure spaces via derivations and integration by parts (2014). Submitted, arXiv:1409.5620

18. Di Marino, S., Gigli, N., Pasqualetto, E., Soultanis, E.: Infinitesimal Hilbertianity of locally CAT $(\kappa)$ -spaces (2018). J. Geom. Anal. (2020). https://doi.org/10.1007/s12220-020-00543-7

19. Di Marino, S., Lučić, D., Pasqualetto, E.: A short proof of the infinitesimal Hilbertianity of the weighted Euclidean space. Comptes Rendus Math. 358, 817-825 (2020)

20. Di Marino, S., Speight, G.: The $p$-weak gradient depends on $p$. Proc. Am. Math. Soc. 143, 5239-5252 (2015)

21. Federer, H., Fleming, W.H.: Normal and integral currents. Ann. Math. 72, 458-520 (1960)

22. Fragalà, I., Mantegazza, C.: On some notions of tangent space to a measure. Proc. R. Soc. Edinb. 129A, 331-342 (1999)

23. Gigli, N.: On the differential structure of metric measure spaces and applications. Mem. Am. Math. Soc. 236, vi+91 (2015)

24. Gigli, N.: Nonsmooth differential geometry-an approach tailored for spaces with Ricci curvature bounded from below. Mem. Am. Math. Soc. 251, 161 (2017)

25. Gigli, N.: Lecture notes on differential calculus on RCD spaces. Publ. RIMS Kyoto Univ. 54 (2018)

26. Gigli, N., Han, B.-X.: Sobolev spaces on warped products. J. Funct. Anal. 275, 2059-2095 (2018)

27. Gigli, N., Pasqualetto, E.: Behaviour of the reference measure on RCD spaces under charts. To appear in Commun. Anal. Geom. https://www.arXiv:1607.05188 
28. Gol'dshtein, V., Troyanov, M.: Axiomatic theory of Sobolev spaces. Expos. Math. 19, 289-336 (2001)

29. Hajłasz, P.: Sobolev spaces on an arbitrary metric space. Potential Anal. 5, 403-415 (1996)

30. Heinonen, J., Koskela, P., Shanmugalingam, N., Tyson, J.: Sobolev Spaces on Metric Measure Spaces: An Approach Based on Upper Gradients, p. 1. Cambridge University Press, New York (2015)

31. Kell, M., Mondino, A.: On the volume measure of non-smooth spaces with Ricci curvature bounded below. Ann. Sc. Norm. Super. Pisa Cl. Sci. (5) 18, 593-610 (2018)

32. Louet, J.: Problèmes de transport optimal avec pénalisation en gradient. Thèse de doctorat dirigée par Santambrogio Filippo, Mathématiques Paris 11 (2014)

33. Louet, J.: Some results on Sobolev spaces with respect to a measure and applications to a new transport problem. J. Math. Sci. 196, 152-164 (2014)

34. Lučić, D., Pasqualetto, E.: The Serre-Swan theorem for normed modules. Rendiconti del Circolo Matematico di Palermo Series 2(68), 385-404 (2019)

35. Pasqualetto, E.: Testing the Sobolev property with a single test plan (2020). Submitted, arXiv $: 2006.03628$

36. Preiss, D.: Geometry of measures in $\mathbb{R}^{n}$ : distribution, rectifiability, and densities. Ann. Math. 125, 537-643 (1987)

37. Schioppa, A.: Poincaré inequalities for mutually singular measures. Anal. Geom. Metr. Spaces 4, 40-45 (2015)

38. Shanmugalingam, N.: Newtonian spaces: an extension of Sobolev spaces to metric measure spaces. Rev. Mat. Iberoamericana 16, 243-279 (2000)

39. Simon, L.: Lectures on geometric measure theory. In: Proceedings of the Centre for Mathematical Analysis. Australian National University (1984)

40. Zhikov, V.V.: On an extension of the method of two-scale convergence and its applications. Sb. Math. 191, 973-1014 (2000)

41. Zhikov, V.V.: Homogenization of elasticity problems on singular structures. Izv. Math. 66, 299-365 (2002)

Publisher's Note Springer Nature remains neutral with regard to jurisdictional claims in published maps and institutional affiliations. 